\title{
Layers in the Presence of Conservation Laws
}

\author{
Alin Pogan and Arnd Scheel \\ University of Minnesota \\ School of Mathematics \\ 206 Church St. S.E. \\ Minneapolis, MN 55455, USA
}

\begin{abstract}
We study standing layers in systems where a reaction-diffusion equation couples to a scalar conservation law. Our results give spectral stability and instability results depending only on relative monotonicity of the two components of the system. We also prove the robustness of layers and their stability properties. Our results classify stability properties of layers in most such systems. Our method is based on tracking the point spectrum during a homotopy to a simple, decoupled system. Main difficulty is the possibility of eigenvalues disappearing in a branch point of the essential spectrum. This phenomenon is investigated using a Lyapunov-Schmidt reduction method on exponentially weighted spaces combined with a matching procedure for the far-field. ${ }^{1}$
\end{abstract}

\section{Introduction}

In this paper we study the stability of heteroclinic steady states (layers) in systems where a scalar conservation law is coupled to a scalar reaction-diffusion equation. More precisely, we consider

$$
\left\{\begin{array}{l}
u_{t}=\left[a(u) u_{x}-b(u) v_{x}\right]_{x} \\
v_{t}=v_{x x}+\delta u+g(v)
\end{array}\right.
$$

on the real line $x \in \mathbb{R}$. Here $a, b, g \in C^{3}(\mathbb{R}), \delta \in \mathbb{R}$. Moreover, $a$ is uniformly elliptic, that is, $a(u) \geq a_{0}>0$ for all $u \in \mathbb{R}$. Note that this system conserves mass $\int u$ with suitable decay conditions at $x= \pm \infty$.

Such systems arise in a number of physical, biological, and chemical applications; $[1,3,4,10,11$, $15,16,27]$. A fairly simple model arises in the theory of chemical conversion equations $C \longleftrightarrow E$ in a closed reactor. Assuming a conversion rate $f(c, e)=\delta c+g(e)$ for the concentrations $c=[C]$ and $e=[E]$, with linear decay of $C$ and nonlinear (autocatalytic) production of $E$, we find a system

$$
\left\{\begin{array}{l}
c_{t}=c_{x x}-\delta c-g(e), \\
e_{t}=d e_{x x}+\delta c+g(e) .
\end{array}\right.
$$

Here, the diffusion rate of the species $C$ has been normalized to one and $d$ is the diffusion rate of the chemical species $E$. Making the change of variables $u=c+e, v=e$, and rescaling $x$,

${ }^{1}$ AMS MSC 2000 Mathematics Subject Classification: 37L15

Keywords: heteroclinic solutions, conservation law, stability, Lyapunov-Schmidt reduction. 
one can rewrite (1.2) in the form (1.1) with constant $a(u)=1$ and $b(u)=1-d$. Systems of this type arise frequently in biology, chemistry, and combustion; see $[3,10,16,14]$ for some examples.

More directly, systems of the form (1.1) appear in the theory of thermodynamic phase transitions, such as the phase-field system

$$
\left\{\begin{array}{l}
\tau \varphi_{t}=d_{\varphi} \varphi_{x x}+\frac{1}{2}\left(\varphi-\varphi^{3}\right)+2 w \\
w_{t}+\frac{1}{2} \ell \varphi_{t}=d_{w} w_{x x}
\end{array}\right.
$$

Here $w$ denotes the temperature, $\varphi$ is an order parameter which is defined such that $\varphi=-1$ corresponds to the solid phase and $\varphi=1$ corresponds to the liquid phase. In addition, $d_{w}$ characterizes heat flux, the constant $d_{\varphi}$ accounts for interfacial energy, and $\ell$ is the latent heat; see for instance $[1,4]$ and references therein. One readily converts (1.3) into the form (1.1) by first rescaling $t, x$ so that $\tau=d_{\varphi}=1$, and then employing the linear change of variables $u=w+\frac{\ell}{2} \varphi$ and $v=\varphi$, which gives $a(u)=d_{w}, b(u)=-\frac{\ell}{2} d_{w}$, and $g(v)=\frac{1}{2}\left(v-v^{2}\right)-\ell v$.

More general forms of the functions $a$ and $b$ appear in models for chemotactic phenomena. We let $u$ measure the concentration of a cell population, and $v$ the concentration of a chemical produced by the bacteria. Following [12], the system

$$
\left\{\begin{array}{l}
u_{t}=\left[\left(q(u)-u q^{\prime}(u)\right) u_{x}-\chi u q(u) v_{x}\right]_{x} \\
v_{t}=v_{x x}+\delta u-\beta v .
\end{array}\right.
$$

describes chemotactic behavior with chemotactic sensitivity $\chi$ and a density-dependent probability factor $q(\cdot)$ that measures the probability of a cell to find space at a neighboring location. The parameter $\delta$ and $\beta$ measure linear rates of production and decay for the chemical.

From a point of view of pattern formation, systems of the form (1.1) are richer than scalar reaction-diffusion equations, but much simpler than systems of, say, two reaction-diffusion equations. Roughly speaking, precisely the monotone solutions are stable in scalar reactiondiffusion equations [7], while systems allow for a variety of stable spatia-temporal oscillations. In previous work $[22,23]$, we showed that spikes are always unstable in systems of the form (1.1), similar to the scalar reaction-diffusion setting. On the other hand, spatially periodic patterns can be stable in systems of the form (1.1) when considered with periodic boundary conditions $[5,18,19]$, although they are always unstable in scalar equations.

Our present paper is concerned with the stability of layer solutions to (1.1). We will see below that in our case the existence problem is essentially equivalent to the scalar case after integrating the $u$-equation. As a consequence, (1.1) exhibits the same types of patterns as scalar equations, in particular it may possess heteroclinic solutions. Stability properties are somewhat more subtle: while layers are always stable in scalar reaction-diffusion equations, we will see that layers can destabilize in a drift-bifurcation similar to the one observed in reaction-diffusion systems; see $[2,8,13,25]$.

In order to prepare for the statements of our main results, we give a short outline of the construction of layers. More details will be provided in Section 2. To find a layer solution we 
first look at the steady state system:

$$
\left\{\begin{array}{l}
{\left[a(u) u_{x}-b(u) v_{x}\right]_{x}=0} \\
v_{x x}+\delta u+g(v)=0
\end{array}\right.
$$

One can integrate the first equation and recast it as a differential equation for $u$ in terms of $v$. We write $u=\Phi(v)$ for solutions of that ordinary differential equation. One inserts this expression into the second equation of (1.5) and obtains a non-linear second-order pendulum equation in $v$. This equation can have homoclinic, periodic or heteroclinic (layers) solutions.

We will focus on the case when this equation possess a heteroclinic solution $v^{*}$. We note that $v^{*}$ is necessarily monotone and write $v^{ \pm}=\lim _{x \rightarrow \pm \infty} v^{*}(x) \in \mathbb{R}$. Moreover, since $\Phi$ is the solution of an autonomous differential equation, we infer that $\Phi$ is monotone or constant, which implies that the $u$-component of the layer, $u^{*}=\Phi\left(v^{*}\right)$ is strictly monotone or constant. Also, $u^{ \pm}=\lim _{x \rightarrow \pm \infty} u^{*}(x)=\Phi\left(v^{ \pm}\right) \in \mathbb{R}$.

We will next formulate the main result of this paper. It characterizes stability of layers in a large subclass of (1.1). Stability here is referred to as spectral stability. Our notion of stability refers to the spectrum of the linearization of (1.1) at a layer $\left(u^{*}, v^{*}\right)$,

$$
\frac{\mathrm{d}}{\mathrm{d} t}\left(\begin{array}{l}
u \\
v
\end{array}\right)=\mathcal{L}\left(\begin{array}{l}
u \\
v
\end{array}\right)
$$

where

$$
\mathcal{L}=\left[\begin{array}{cc}
\partial_{x}\left(a\left(u^{*}\right) \partial_{x}+a^{\prime}\left(u^{*}\right) u_{x}^{*}-b^{\prime}\left(u^{*}\right) v_{x}^{*}\right) & -\partial_{x}\left(b\left(u^{*}\right) \partial_{x}\right) \\
\delta & \partial_{x}^{2}+g^{\prime}\left(v^{*}\right)
\end{array}\right]
$$

We can view the differential expression $\mathcal{L}$ as a densely defined, closed operator on various function spaces like $B U C\left(\mathbb{R}, \mathbb{C}^{2}\right), L^{2}\left(\mathbb{R}, \mathbb{C}^{2}\right)$ or $L_{\eta}^{2}\left(\mathbb{R}, \mathbb{C}^{2}\right)$ with respective domain $B U C^{2}\left(\mathbb{R}, \mathbb{C}^{2}\right)$, $H^{2}\left(\mathbb{R}, \mathbb{C}^{2}\right)$ or $H_{\eta}^{2}\left(\mathbb{R}, \mathbb{C}^{2}\right)$; see the end of this section for definitions of spaces. Since $\mathcal{L}$ is a lowerorder perturbation of a diffusion operator, it generates an analytic semigroup with maximal regularity properties so that the nonlinear evolution problem is in fact well-posed; see for instance [17] for the relevant semigroup machinery. We say that a layer is spectrally stable if the spectrum of $\mathcal{L}$ is contained in the closed left half plane, $\{\lambda \in \mathbb{C}: \operatorname{Re} \lambda \leq 0\}$. It turns out that spectral stability in $L^{2}$ is equivalent to spectral stability in $B U C$. On the other hand, spectral instability implies nonlinear instability; see [9].

We will see in Section 3 that the essential spectrum is contained in the closed left half plane if and only if $g^{\prime}\left(v^{ \pm}\right) \leq 0$; see Lemma 3.4. We therefore assume in the following that $g^{\prime}\left(v^{ \pm}\right)<0$.

Theorem 1.1. Assume $\left(u^{*}, v^{*}\right)$ is an exponentially localized layer of (1.1) for $\delta=\delta_{0}$, that is

$$
\left|\left(u^{*}-u^{ \pm}, v^{*}-v^{ \pm}\right)(x)\right| \leq C \mathrm{e}^{-\eta^{*}|x|}, \quad \text { for all } \quad x \in \mathbb{R}_{ \pm}
$$

for some constants $C, \eta^{*}>0$. Also, suppose that $g^{\prime}\left(v^{ \pm}\right)<0$. Then, the following assertions hold true:

(i) If $\delta_{0}>0$, and $\left(u^{+}-u^{-}\right)\left(v^{+}-v^{-}\right)>0$, then the layer $\left(u^{*}, v^{*}\right)$ is spectrally stable in $L^{2}$ or BUC; 
(ii) If $\left(u^{+}-u^{-}\right)\left(v^{+}-v^{-}\right) \delta_{0}<0$, then the layer $\left(u^{*}, v^{*}\right)$ is unstable in $L^{2}$ or BUC.

(iii) If $\delta_{0}=0$ or $u^{+}=u^{-}$, then the layer $\left(u^{*}, v^{*}\right)$ is spectrally stable in $L^{2}$ or BUC.

Related to the temporal stability question is the robustness question, that is, the question whether layers persist as solutions of (1.5) when parameters in the system are varied. We say that a layer is robust if for nearby values of parameters the system possesses a layer that is close to the original layer in $B C^{1}\left(\mathbb{R}, \mathbb{R}^{2}\right)$. If a layer is robust, we say that the stability properties of a layer are robust if the layer is spectrally stable (or unstable) for all perturbations.

Theorem 1.2. Assume $\left(u^{*}, v^{*}\right)$ is an exponentially localized, stable layer of (1.1) for $\delta=\delta_{0}$. Then, the following assertions hold true:

(i) If $\delta_{0} \neq 0$, the layer is robust under $\varepsilon$-perturbations of $a, b, g \in C^{3}$;

(ii) If $\delta_{0}=0$, the layer is robust under $\delta$-perturbations if and only if $\int_{\mathbb{R}} u^{*} v_{x}^{*}=0$;

(iii) Suppose $\delta_{0}>0$. Then stability properties of layers are robust under $\varepsilon$-perturbations of $a, b, g$ if and only if $u^{+} \neq u^{-}$;

(iv) Suppose $u_{+} \neq u_{-}$. Then the stability properties of layers are not robust under $\delta-$ perturbations from $\delta_{0}=0$.

Remark 1.3. Both theorems apply to the examples (1.2)-(1.4). Choosing for instance $g(e)=$ $e\left(1-e^{2}\right)$ and $c+e \equiv \mu=0$ in (1.2), one finds layers $e(x)=-e(-x)$ as solutions to de $e_{x x}-$ $\delta e+g(e)=0$. Similarly, (1.3) possesses layers with $w \equiv 0, \varphi(x)=\tanh \left(x / \sqrt{d_{\varphi}}\right)$. The chemotaxis system possesses layers for certain functions $q$. To see this, note that we can scale $\beta=\delta=1$ and set $q(u)=u \exp (-u+\varepsilon \Phi)$, with some function $\Phi$ to be determined. We then find, similarly to the discussion above, $\frac{\mathrm{d} v}{\mathrm{~d} u}=(u-\varepsilon \Phi(u ; \varepsilon))^{\prime}$, so that $v(u)=u-\varepsilon \Phi(u ; \varepsilon)$. Choosing $\Phi$ appropriately allows us to realize, for instance, $u(v)=v+\varepsilon \sin v$, which in turn gives layers for the $v$-equation, $v_{x x}+u(v)-v=0$, connecting $v=0$ to $v=2 \pi$.

In all those cases, Theorem 1.1 ensures spectral stability of the layer.

We will see in Section 2 that the proof of Theorem 1.2 is fairly standard, given Theorem 1.1.

The main difficulty in proving the results in Theorem 1.1 is the presence of essential spectrum on the imaginary axis. Essential spectrum is caused by the fact that we are considering an unbounded domain $x \in \mathbb{R}$. Since the first equation is in conservation form, $\lambda=0$ automatically contributes to the essential spectrum through a constant adjoint eigenmode. On the other hand, essential spectrum on the imaginary axis prevents us from immediately concluding nonlinear stability.

Our results give in fact a more precise characterization of spectral properties of the linearization at layers. We calculate expansions of an Evans function so that we can describe poles of the resolvent. Moreover, our method gives algebraic and geometric multiplicities of eigenvalues in the essential spectrum in a natural fashion. 
We next outline the key steps in our proof. First, we compute the essential spectrum of the linearization $\mathcal{L}$ and restrict to the case $g^{\prime}\left(v^{ \pm}\right)<0$, which implies stability of the essential spectrum. In this case, we use a homotopy to a triangular system to track the eigenvalues with positive real part. The homotopy is chosen such that the layer $\left(u^{*}, v^{*}\right)$ and the stability of the essential spectrum do not depend on the homotopy parameter. In the last and crucial step, we track eigenvalues as they cross the imaginary axis, in particular as they emerge from or disappear into the essential spectrum at $\lambda=0$. For this, we follow eigenvalues into the essential spectrum using a Lyapunov-Schmidt reduction method on exponentially weighted spaces. In these spaces, the linearization turns out to possess a negative Fredholm index. We compensate for this negative index by augmenting the system with parametrized asymptotic expansions. This yields a generalized eigenvalue problem that is Fredholm index zero in a neighborhood of the origin, so that eigenvalues of this problem correspond to eigenvalues of our linearization for $\operatorname{Re} \lambda>0$. Similar approaches have been used before in [22, 23, 24, 26].

Outline: The paper is organized as follows. In Section 2 we study the problem of layer robustness in two situations: under $\varepsilon$-perturbations of $a, b$ and $g$, and second, under $\delta$-perturbations from $\delta_{0}=0$. Next, in Section 3 we compute the essential spectrum of $\mathcal{L}$. In the next four sections we construct the homotopy, prove the existence and the analyticity of a bifurcation function that detects the eigenvalues of $\mathcal{L}$, and finally compute its expansions. Theorem 1.1 and Theorem 1.2 are proven in Section 8 and Section 9, respectively.

Notations: For an operator $T$ on a Hilbert space $X$ we use $T^{*}, \operatorname{dom}(T), \operatorname{ker} T, \operatorname{im} T, \sigma(T)$, $\rho(T)$ and $T_{\mid Y}$ to denote the adjoint, domain, kernel, range, spectrum, resolvent set and the restriction of $T$ on a subspace $Y$ of $X$. We divide the spectrum of $T$ into two disjoint sets: $\sigma_{\text {point }}(T)$, the union of eigenvalues $\lambda$ for which $T-\lambda$ is Fredholm with index 0 , and $\sigma_{\text {ess }}(T)$ its complement in $\sigma(T)$. The Morse index of a hyperbolic matrix $A$, denoted $i(A)$, is the dimension of its unstable subspace, which is the generalized eigenspace associated with all eigenvalues $\lambda$ of $A$ that have Re $\lambda>0$. The usual Lebesgue spaces, the space of bounded uniformly continuous functions and the weighted Lebesgue spaces of vector valued functions are denoted by $L^{p}\left(\mathbb{R}, \mathbb{C}^{N}\right), B U C\left(\mathbb{R}, \mathbb{C}^{N}\right)$ and $L^{p}\left(\mathbb{R}, \mathbb{C}^{N} ; \omega(x) d x\right)$ respectively. If $\omega(x)=e^{2 \eta|x|}$ for all $x \in \mathbb{R}$ we denote the $L^{p}$-weighted space by $L_{\eta}^{p}\left(\mathbb{R}, \mathbb{C}^{N}\right)$. If $h=h(u)$ is a smooth function and $u^{*}$ is the $u$-component of a layer $\left(u^{*}, v^{*}\right)$, we use the notations $h^{*}:=h\left(u^{*}\right)$ and $h^{ \pm}:=h\left(u^{ \pm}\right)$, where $u^{ \pm}=\lim _{x \rightarrow \pm \infty} u^{*}(x)$.

Acknowledgment. The authors gratefully acknowledges support by the National Science Foundation under grant DMS-0806614.

\section{Robustness of Layers}

In this section we explain how one can find layer solutions in the general model (1.1) and study their robustness under perturbations of the "parameters" $a, b, g$ and $\delta$.

We will work under the assumptions of Theorem 1.2. In particular, we assume that (1.5) has an exponentially localized layer solution for $\delta=\delta_{0}$, where $\delta_{0}$ is a fixed value. We remark that since a layer solution is bounded, we can assume without loss of generality that $b$ is bounded. 
In what follows we will study the robustness problem for two separate cases: first, robustness under $\varepsilon$-perturbations of $a, b$ and $g$ and second, robustness under $\delta$-perturbations from $\delta_{0}=0$. We start by rewriting (1.5) as a first order system by making the substitution $w=v_{x}$ and $\psi=a(u) u_{x}-b(u) v_{x}$. We obtain the system

$$
\left\{\begin{array}{l}
u_{x}=\frac{b(u)}{a(u)} w+\frac{\psi}{a(u)} \\
v_{x}=w \\
w_{x}=-\delta u-g(v) \\
\psi_{x}=0
\end{array}\right.
$$

This system can be written in the form $(u, v, w, \psi)_{x}^{\mathrm{T}}=F(u, v, w, \psi)$, where $F: \mathbb{R}^{4} \rightarrow \mathbb{R}^{4}$ is defined by $F(u, v, w, \psi)=\left(\frac{b(u)}{a(u)} w+\frac{\psi}{a(u)}, w,-\delta u-g(v), 0\right)^{\mathrm{T}}$. We note that if $(u, v, w, \psi)^{\mathrm{T}}$ is an equilibrium of $F$ then $w=\psi=0$, which allows us to restrict system (2.1) to the case $\psi=0$, that is,

$$
\left\{\begin{array}{l}
u_{x}=\frac{b(u)}{a(u)} w, \\
v_{x}=w \\
w_{x}=-\delta u-g(v) .
\end{array}\right.
$$

The next step is to take advantage of the conserved quantities in (2.2) to make a change of variables. We denote by $\varphi(\cdot, \mu)$ the solution of the Cauchy problem

$$
\left\{\begin{array}{l}
u^{\prime}=\frac{b(u)}{a(u)}, \\
u(0)=\mu .
\end{array}\right.
$$

Note that one can formally derive (2.3) from (2.2) by taking the quotient of the first two equations and thus obtaining an equation for $u^{\prime}=\frac{\mathrm{d} u}{\mathrm{~d} v}$. We will make this relation rigorous in the following.

Lemma 2.1. The following assertions are true:

(i) $\partial_{\mu} \varphi(v, \mu)=\exp \left(\int_{0}^{v}\left(\frac{b}{a}\right)^{\prime}(\varphi(w, \mu)) \mathrm{d} w\right)$ for all $v, \mu \in \mathbb{R}$;

(ii) Given $u, v$, the equation $u=\varphi(v, \mu)$ has a unique solution $\left.\mu=\mu^{*}(u, v)\right)$;

(iii) If $(u, v, w)$ satisfies $(2.2)$ then $\mu^{*}(u, v)$ is a conserved quantity of (2.2).

Proof. (i) From the definition of $\varphi(\cdot, \mu)$ in $(2.3)$, by differentiating with respect to $\mu$, we infer that for any fixed $\mu \in \mathbb{R}$ the function $\partial_{\mu} \varphi(\cdot, \mu)$ satisfies the Cauchy problem

$$
\left\{\begin{array}{l}
u^{\prime}=\left[\left(\frac{b}{a}\right)^{\prime}(\varphi(v, \mu))\right] u, \\
u(0)=1
\end{array}\right.
$$

Solving this first order linear Cauchy problem, we immediately find the desired formula. Assertion (ii) follows by noticing the flow property, $\varphi(-v, \varphi(v, \mu))=\mu$, so that $\mu_{*}(u, v)=\varphi(-v, u)$. (iii) Assume that $(u, v, w)$ satisfies (2.2). Differentiating the equation $u=\varphi\left(v, \mu^{*}(u, v)\right)$ with respect to $u$ and $v$ respectively, we obtain that

$$
1=\partial_{\mu} \varphi\left(v, \mu^{*}(u, v)\right) \partial_{u} \mu^{*}(u, v)
$$




$$
0=\partial_{v} \varphi\left(v, \mu^{*}(u, v)\right)+\partial_{\mu} \varphi\left(v, \mu^{*}(u, v)\right) \partial_{v} \mu^{*}(u, v) .
$$

Solving for $\partial_{u} \mu^{*}$ and $\partial_{v} \mu^{*}$ in (2.5) and using the definition of $\varphi(\cdot, \mu)$ in $(2.3)$ we obtain that

$$
\partial_{v} \mu^{*}(u, v)=-\frac{\partial_{v} \varphi\left(v, \mu^{*}(u, v)\right)}{\partial_{\mu} \varphi\left(v, \mu^{*}(u, v)\right)}=-\frac{b\left(\varphi\left(v, \mu^{*}(u, v)\right)\right)}{a\left(\varphi\left(v, \mu^{*}(u, v)\right)\right)} \partial_{u} \mu^{*}(u, v)=-\frac{b(u)}{a(u)} \partial_{u} \mu^{*}(u, v) .
$$

Next, using (2.6) we compute

$$
\begin{aligned}
\frac{\mathrm{d}}{\mathrm{d} x}\left[\mu^{*}(u(x), v(x))\right] & =\partial_{u} \mu^{*}(u(x), v(x)) u^{\prime}(x)+\partial_{v} \mu^{*}(u(x), v(x)) v^{\prime}(x) \\
& =\partial_{u} \mu^{*}(u(x), v(x)) \frac{b(u(x))}{a(u(x))} w(x)-\frac{b(u(x))}{a(u(x))} \partial_{u} \mu^{*}(u(x), v(x)) w(x)=0 .
\end{aligned}
$$

This lemma shows that we can make the substitution $\mu=\mu^{*}(u, v)$ in $(2.2)$ to obtain the system

$$
\left\{\begin{array}{l}
v_{x}=w \\
w_{x}=-\delta \varphi(v, \mu)-g(v), \\
\mu_{x}=0
\end{array}\right.
$$

Since (1.5) has a layer solution $\left(u_{0}^{*}, v_{0}^{*}\right)$ for $\delta=\delta_{0}$, we have that (2.7) has a layer solution, $v_{0}^{*}$ at $\mu=\mu_{0}$ and $\delta=\delta_{0}$. We denote by $v_{0}^{ \pm}=\lim _{x \rightarrow \pm \infty} v_{0}^{*}(x)$. Note that the equation for $v$ is a nonlinear pendulum equation of the form $v_{x x}+H(v, \mu)=0$, where we define $H: \mathbb{R}^{2} \rightarrow \mathbb{R}$ by

$$
H(v, \mu)=\delta_{0} \varphi(v, \mu)+g(v) .
$$

Lemma 2.2. The existence of an exponentially localized layer solution implies the following set of properties of the function $H$ and the limits $v_{0}^{ \pm}$

- equilibria: $H\left(v_{0}^{ \pm}, \mu_{0}\right)=0$;

- hyperbolicity: $\partial_{v} H\left(v_{0}^{ \pm}, \mu_{0}\right)<0$;

- equal-area: $\int_{v_{0}^{-}}^{v^{+}} H\left(v, \mu_{0}\right) \mathrm{d} v=0$.

Proof. The first property follows readily from the convergence of $v_{0}^{*}$. Exponential convergence implies hyperbolicity of the equilibria $v_{0}^{ \pm}$. Indeed, for non-hyperbolic equilibria, solutions near $v_{0}^{ \pm}$either oscillate or decay algebraically, as an direct solution to the integrable Hamiltonian equation shows. Lastly, the equal area condition is a consequence of conservation of energy $\frac{1}{2} v_{x}^{2}+\int^{v} H(w, \mu) \mathrm{d} \mu$ along the layer solution $v_{0}^{*}$.

First, we treat the case when $\delta_{0} \neq 0$ and study the robustness of layers under $\varepsilon$-perturbations of $a, b$ and $g$. Let $\varepsilon_{0}>0$, and $\tilde{a}, \tilde{b}, \tilde{g} \in C^{3}\left(\mathbb{R} \times\left(-\varepsilon_{0}, \varepsilon_{0}\right), \mathbb{R}\right)$ be families of functions such that $\tilde{a}(\cdot, 0)=a, \tilde{b}(\cdot, 0)=b$ and $\tilde{g}(\cdot, 0)=g$ and consider the system

$$
\left\{\begin{array}{l}
u_{x}=\frac{\tilde{b}(u, \varepsilon)}{\tilde{a}(u, \varepsilon)} w \\
v_{x}=w \\
w_{x}=-\delta_{0} u-\tilde{g}(v, \varepsilon) .
\end{array}\right.
$$


System (2.9) is a smooth perturbation of $(2.2)$. We define $\tilde{\varphi}(\cdot, \mu, \varepsilon)$ as the solution of the Cauchy problem

$$
\left\{\begin{array}{l}
u^{\prime}=\frac{\tilde{b}(u, \varepsilon)}{\tilde{a}(u, \varepsilon)}, \\
u(0)=\mu
\end{array}\right.
$$

Using Lemma 2.1 we can construct $\tilde{\mu}^{*}(u, v, \varepsilon)$ that solves the equation $u=\varphi(v, \mu, \varepsilon)$. Furthermore, making the substitution $\mu=\tilde{\mu}^{*}(u, v, \varepsilon)$ we can transform (2.9) in the equivalent form

$$
\left\{\begin{array}{l}
v_{x}=w \\
w_{x}=-\delta_{0} \tilde{\varphi}(v, \mu, \varepsilon)-\tilde{g}(v, \varepsilon), \\
\mu_{x}=0
\end{array}\right.
$$

Let $\tilde{H}: \mathbb{R}^{3} \rightarrow \mathbb{R}$ be the function defined by

$$
\tilde{H}(v, \mu, \varepsilon)=\tilde{g}(v, \varepsilon)+\delta_{0} \tilde{\varphi}(v, \mu, \varepsilon) .
$$

To show the $\varepsilon$-robustness of the layer we need:

(1) $\varepsilon$-robustness of the equilibria $v_{0}^{ \pm}$: There exist smooth functions $\mu(\varepsilon)$ and $v^{ \pm}(\varepsilon)$ such that

(a) $\mu(0)=\mu_{0}$ and $v^{ \pm}(0)=v_{0}^{ \pm}$;

(b) locally, the equation $\tilde{H}(v, \mu(\varepsilon), \varepsilon)=0$ has the unique solution $v=v^{ \pm}(\varepsilon)$;

(2) $\varepsilon$-robustness of the equal area condition: $\int_{v^{-}(\varepsilon)}^{v^{+}(\varepsilon)} \tilde{H}(v, \mu(\varepsilon), \varepsilon) \mathrm{d} v=0$.

To see that this indeed guarantees persistence of the layer, notice that the stable and unstable manifolds of $v_{0}^{ \pm}$depend smoothly on the parameter $\varepsilon$. Given the equal area condition, they both lie in the same level set of the Hamiltonian $\frac{1}{2} v_{x}^{2}+H(v)$ for all small $\varepsilon$. The intersection therefore persists within this level set which is a smooth curve away from the equilibrium.

In the next lemma we prove one of the main results of this section.

Lemma 2.3. Assume (2.7) has a layer $v_{0}^{*}$ at $\mu=\mu_{0}$ and $\delta=\delta_{0} \neq 0$. Then the layer is robust under $\varepsilon$-perturbations of $a, b$ and $g$.

Proof. First, we will show that we can locally solve the equation $\tilde{H}(v, \mu, \varepsilon)=0$ for $v$. Since

$$
\begin{aligned}
& \tilde{H}\left(v_{0}^{ \pm}, \mu_{0}, 0\right)=H\left(v_{0}^{ \pm}, \mu_{0}\right)=0 ; \\
& \partial_{v} \tilde{H}\left(v_{0}^{ \pm}, \mu_{0}, 0\right)=\partial_{v} H\left(v_{0}^{ \pm}, \mu_{0}\right)<0,
\end{aligned}
$$

we find from the Implicit Function Theorem that there are two $C^{3}$-functions $\tilde{v}^{ \pm}(\mu, \varepsilon)$ such that

(i) $\tilde{v}^{ \pm}\left(\mu_{0}, 0\right)=v_{0}^{ \pm}$;

(ii) In a neighborhood of $\left(v_{0}^{ \pm}, \mu_{0}\right)$, respectively, the equation $\tilde{H}(v, \mu, \varepsilon)=0$ has the unique solution $v=\tilde{v}^{ \pm}(\mu, \varepsilon)$. 
Next, we define the area function $A: \mathbb{R}^{2} \rightarrow \mathbb{R}$ by

$$
A(\mu, \varepsilon)=\int_{\tilde{v}^{-}(\mu, \varepsilon)}^{\tilde{v}^{+}(\mu, \varepsilon)} \tilde{H}(v, \mu, \varepsilon) \mathrm{d} v .
$$

We will show that we can locally solve the equation $A(\mu, \varepsilon)=0$ for $\mu$. Using condition (i) above we compute

$$
A\left(\mu_{0}, 0\right)=\int_{\tilde{v}^{-}\left(\mu_{0}, 0\right)}^{\tilde{v}^{+}\left(\mu_{0}, 0\right)} \tilde{H}\left(v, \mu_{0}, 0\right) \mathrm{d} v=\int_{v_{0}^{-}}^{v_{0}^{+}} H\left(v, \mu_{0}\right) \mathrm{d} v=0 .
$$

Similarly, using condition (ii) we compute

$$
\begin{aligned}
\partial_{\mu} A(\mu, \varepsilon)= & \int_{\tilde{v}^{-}(\mu, \varepsilon)}^{\tilde{v}^{+}(\mu, \varepsilon)} \partial_{\mu} \tilde{H}(v, \mu, \varepsilon) \mathrm{d} v+\tilde{H}\left(\tilde{v}^{+}(\mu, \varepsilon), \mu, \varepsilon\right) \partial_{\mu} \tilde{v}^{+}(\mu, \varepsilon) \\
& \quad-\tilde{H}\left(\tilde{v}^{-}(\mu, \varepsilon), \mu, \varepsilon\right) \partial_{\mu} \tilde{v}^{-}(\mu, \varepsilon) \\
= & \int_{\tilde{v}^{-}(\mu, \varepsilon)}^{\tilde{v}^{+}(\mu, \varepsilon)} \delta_{0} \partial_{\mu} \tilde{\varphi}(v, \mu, \varepsilon) \mathrm{d} v .
\end{aligned}
$$

From (2.14) and Lemma 2.1(i) we have that

$$
\partial_{\mu} A\left(\mu_{0}, 0\right)=\delta_{0} \int_{v_{0}^{-}}^{v_{0}^{+}} \partial_{\mu} \tilde{\varphi}(v, \mu, \varepsilon) \mathrm{d} v \neq 0 .
$$

Using the Implicit Function Theorem again, we infer that there exists a smooth function $\mu(\varepsilon)$ such that

(iii) $\mu(0)=\mu_{0}$;

(iv) locally, the equation $A(\mu, \varepsilon)=0$ has the unique solution $\mu=\mu(\varepsilon)$.

Next, we define $v^{ \pm}(\varepsilon)=\tilde{v}^{ \pm}(\mu(\varepsilon), \varepsilon)$. From (ii) we have that locally the equation $\tilde{H}(v, \mu(\varepsilon), \varepsilon)=$ 0 has the unique solution $v=v^{ \pm}(\varepsilon)$. From (iv) we obtain that

$$
\int_{v^{-}(\varepsilon)}^{v^{+}(\varepsilon)} \tilde{H}(v, \mu(\varepsilon), \varepsilon) \mathrm{d} v=A(\mu(\varepsilon), \varepsilon)=0
$$

proving the lemma.

Next, we will compute the leading order term in the difference $u^{+}(\varepsilon)-u^{-}(\varepsilon)$, where $u^{ \pm}(\varepsilon)=$ $\tilde{\varphi}\left(v^{ \pm}(\varepsilon), \mu(\varepsilon), \varepsilon\right)$.

Lemma 2.4. The following assertions are true:

$$
\begin{aligned}
& \text { (i) } \partial_{\varepsilon} \tilde{\varphi}\left(v, \mu_{0}, 0\right)=\int_{0}^{v} \frac{\partial_{\mu} \varphi\left(v, \mu_{0}\right)}{\partial_{\mu} \varphi\left(w, \mu_{0}\right)} \partial_{\varepsilon}\left(\frac{\tilde{b}}{\tilde{a}}\right)\left(\varphi\left(w, \mu_{0}\right), 0\right) \mathrm{d} w \quad \text { for all } \quad v \in \mathbb{R} ; \\
& \text { (ii) } \partial_{\varepsilon} A\left(\mu_{0}, 0\right)=\int_{v_{0}^{-}}^{v_{0}^{+}}\left[\partial_{\varepsilon} \tilde{g}(v, 0)+\delta_{0} \partial_{\varepsilon} \tilde{\varphi}\left(v, \mu_{0}, 0\right)\right] \mathrm{d} v ;
\end{aligned}
$$




$$
\begin{aligned}
\text { (iii) }) & \left(u^{+}\right)^{\prime}(0)-\left(u^{-}\right)^{\prime}(0)=\left(\frac{b}{a}\right)\left(u_{0}^{+}\right)\left(v^{+}\right)^{\prime}(0)-\left(\frac{b}{a}\right)\left(u_{0}^{-}\right)\left(v^{-}\right)^{\prime}(0) \\
& +\frac{1}{\delta_{0} \int_{v_{0}^{-}}^{v_{0}^{+}} \partial_{\mu} \varphi\left(v, \mu_{0}\right) \mathrm{d} v} \int_{v_{0}^{-}}^{v_{0}^{+}} \frac{\partial_{\varepsilon}\left(\frac{\tilde{b}}{\tilde{a}}\right)\left(\varphi\left(v, \mu_{0}\right)\right)}{\partial_{\mu} \varphi\left(v, \mu_{0}\right)}\left[\partial_{\mu} \varphi\left(v_{0}^{+}, \mu_{0}\right) \int_{v_{0}^{-}}^{v} \partial_{\mu} \varphi\left(w, \mu_{0}\right) \mathrm{d} w\right. \\
& \left.+\partial_{\mu} \varphi\left(v_{0}^{-}, \mu_{0}\right) \int_{v}^{v_{0}^{+}} \partial_{\mu} \varphi\left(w, \mu_{0}\right) \mathrm{d} w\right] \mathrm{d} v-\frac{\partial_{\mu} \varphi\left(v_{0}^{+}, \mu_{0}\right)-\partial_{\mu} \varphi\left(v_{0}^{-}, \mu_{0}\right)}{\delta_{0} \int_{v_{0}^{-}}^{v_{0}^{+}} \partial_{\mu} \varphi\left(v, \mu_{0}\right) \mathrm{d} v} \int_{v_{0}^{-}}^{v_{0}^{+}} \partial_{\varepsilon} \tilde{g}(v, 0) \mathrm{d} v .
\end{aligned}
$$

Proof. (i) Differentiating in (2.10) with respect to $\varepsilon$ we obtain

$$
\begin{aligned}
{\left[\partial_{\varepsilon} \tilde{\varphi}\left(v, \mu_{0}, 0\right)\right]^{\prime} } & =\partial_{v}\left(\frac{\tilde{b}}{\tilde{a}}\right)\left(\tilde{\varphi}\left(v, \mu_{0}, 0\right), 0\right)\left[\partial_{\varepsilon} \tilde{\varphi}\left(v, \mu_{0}, 0\right)\right]+\partial_{\varepsilon}\left(\frac{\tilde{b}}{\tilde{a}}\right)\left(\varphi\left(v, \mu_{0}\right), 0\right) \\
& =\left(\frac{b}{a}\right)^{\prime}\left(\varphi\left(v, \mu_{0}\right)\right)\left[\partial_{\varepsilon} \tilde{\varphi}\left(v, \mu_{0}, 0\right)\right]+\partial_{\varepsilon}\left(\frac{\tilde{b}}{\tilde{a}}\right)\left(\varphi\left(v, \mu_{0}\right), 0\right) .
\end{aligned}
$$

In addition, since $\tilde{\varphi}\left(0, \mu_{0}, \varepsilon\right)=\mu_{0}$ for all $\varepsilon \in \mathbb{R}$, we infer that $\partial_{\varepsilon} \tilde{\varphi}\left(0, \mu_{0}, 0\right)=0$. Using this initial condition, the fact that $\partial_{\varepsilon} \tilde{\varphi}\left(\cdot, \mu_{0}, 0\right)$ satisfies the first order equation (2.16) and Lemma 2.1(i), we obtain that

$$
\begin{aligned}
\partial_{\varepsilon} \tilde{\varphi}\left(v, \mu_{0}, 0\right) & =\int_{0}^{v}\left[e^{\int_{w}^{v}\left(\frac{b}{a}\right)^{\prime}\left(\varphi\left(s, \mu_{0}\right)\right) \mathrm{d} s}\right] \partial_{\varepsilon}\left(\frac{\tilde{b}}{\tilde{a}}\right)\left(\varphi\left(w, \mu_{0}\right), 0\right) \mathrm{d} w \\
& =\int_{0}^{v} \frac{\partial_{\mu} \varphi\left(v, \mu_{0}\right)}{\partial_{\mu} \varphi\left(w, \mu_{0}\right)} \partial_{\varepsilon}\left(\frac{\tilde{b}}{\tilde{a}}\right)\left(\varphi\left(w, \mu_{0}\right), 0\right) \mathrm{d} w .
\end{aligned}
$$

(ii) Since $\tilde{H}\left(\tilde{v}^{ \pm}(\mu, \varepsilon), \mu, \varepsilon\right)=0$ we obtain that

$$
\begin{aligned}
\partial_{\varepsilon} A(\mu, \varepsilon) & =\int_{\tilde{v}^{-}(\mu, \varepsilon)}^{\tilde{v}^{+}(\mu, \varepsilon)} \partial_{\varepsilon} \tilde{H}(v, \mu, \varepsilon) \mathrm{d} v+\tilde{H}\left(\tilde{v}^{+}(\mu, \varepsilon), \mu, \varepsilon\right) \partial_{\varepsilon} \tilde{v}^{+}(\mu, \varepsilon) \\
& -\tilde{H}\left(\tilde{v}^{-}(\mu, \varepsilon), \mu, \varepsilon\right) \partial_{\varepsilon} \tilde{v}^{-}(\mu, \varepsilon)=\int_{\tilde{v}^{-}(\mu, \varepsilon)}^{\tilde{v}^{+}(\mu, \varepsilon)} \partial_{\varepsilon} \tilde{H}\left(v, \mu_{0}, 0\right) \mathrm{d} v
\end{aligned}
$$

which implies that

$$
\partial_{\varepsilon} A\left(\mu_{0}, 0\right)=\int_{v_{0}^{-}}^{v_{0}^{+}} \partial_{\varepsilon} \tilde{H}\left(v, \mu_{0}, 0\right) \mathrm{d} v=\int_{v_{0}^{-}}^{v_{0}^{+}}\left[\partial_{\varepsilon} \tilde{g}(v, 0)+\delta_{0} \partial_{\varepsilon} \tilde{\varphi}\left(v, \mu_{0}, 0\right)\right] \mathrm{d} v .
$$

(iii) First, we note that since $A(\mu(\varepsilon), \varepsilon)=0$ for all $\varepsilon$ in a neighborhood of 0 , we obtain that

$$
\mu^{\prime}(0)=-\frac{\partial_{\varepsilon} A\left(\mu_{0}, 0\right)}{\partial_{\mu} A\left(\mu_{0}, 0\right)}
$$

Differentiating in the definition of $u^{ \pm}(\varepsilon)$ and using (i), (ii), (2.15) and (2.17), we obtain that

$$
\begin{aligned}
\left(u^{ \pm}\right)^{\prime}(0) & =\partial_{v} \tilde{\varphi}\left(v_{0}^{ \pm}, \mu_{0}, 0\right)\left(v^{ \pm}\right)^{\prime}(0)+\partial_{\mu} \tilde{\varphi}\left(v_{0}^{ \pm}, \mu_{0}, 0\right) \mu^{\prime}(0)+\partial_{\varepsilon} \tilde{\varphi}\left(v_{0}^{ \pm}, \mu_{0}, 0\right) \\
& =\left(\frac{b}{a}\right)\left(\varphi\left(v_{0}^{ \pm}, \mu_{0}\right)\right)\left(v^{ \pm}\right)^{\prime}(0)-\partial_{\mu} \varphi\left(v_{0}^{ \pm}, \mu_{0}\right) \frac{\partial_{\varepsilon} A\left(\mu_{0}, 0\right)}{\partial_{\mu} A\left(\mu_{0}, 0\right)}+\partial_{\varepsilon} \tilde{\varphi}\left(v_{0}^{ \pm}, \mu_{0}, 0\right)
\end{aligned}
$$




$$
\begin{aligned}
& =\left(\frac{b}{a}\right)\left(\varphi\left(v_{0}^{ \pm}, \mu_{0}\right)\right)\left(v^{ \pm}\right)^{\prime}(0)+\int_{0}^{v_{0}^{ \pm}} \frac{\partial_{\mu} \varphi\left(v_{0}^{ \pm}, \mu_{0}\right)}{\partial_{\mu} \varphi\left(v, \mu_{0}\right)} \partial_{\varepsilon}\left(\frac{\tilde{b}}{\tilde{a}}\right)\left(\varphi\left(v, \mu_{0}\right), 0\right) \mathrm{d} v \\
& -\partial_{\mu} \varphi\left(v_{0}^{ \pm}, \mu_{0}\right) \frac{\int_{v_{0}^{-}}^{v_{0}^{+}}\left[\partial_{\varepsilon} \tilde{g}(v, 0)+\delta_{0} \partial_{\varepsilon} \tilde{\varphi}\left(v, \mu_{0}, 0\right)\right] \mathrm{d} v}{\delta_{0} \int_{v_{0}^{-}}^{v_{0}^{+}} \partial_{\mu} \varphi\left(v, \mu_{0}\right) \mathrm{d} v} \\
& =\left(\frac{b}{a}\right)\left(\varphi\left(v_{0}^{ \pm}, \mu_{0}\right)\right)\left(v^{ \pm}\right)^{\prime}(0)+\frac{\partial_{\mu} \varphi\left(v_{0}^{ \pm}, \mu_{0}\right)}{\delta_{0} \int_{v_{0}^{-}}^{v_{0}^{+}} \partial_{\mu} \varphi\left(v, \mu_{0}\right) \mathrm{d} v}\left[-\int_{v_{0}^{-}}^{v_{0}^{+}} \partial_{\varepsilon} \tilde{g}(v, 0) \mathrm{d} v\right. \\
& +\delta_{0} \int_{v_{0}^{-}}^{v_{0}^{+}} \partial_{\mu} \varphi\left(v, \mu_{0}\right) \mathrm{d} v \int_{0}^{v_{0}^{ \pm}} \frac{\partial_{\varepsilon}\left(\frac{\tilde{b}}{\tilde{a}}\right)\left(\varphi\left(v, \mu_{0}\right), 0\right)}{\partial_{\mu} \varphi\left(v, \mu_{0}\right)} \mathrm{d} v \\
& \left.-\delta_{0} \int_{v_{0}^{-}}^{v_{0}^{+}} \partial_{\mu} \varphi\left(v, \mu_{0}\right)\left(\int_{0}^{v} \frac{\partial_{\varepsilon}\left(\frac{\tilde{b}}{\tilde{a}}\right)\left(\varphi\left(w, \mu_{0}\right), 0\right)}{\partial_{\mu} \varphi\left(w, \mu_{0}\right)} \mathrm{d} w\right) \mathrm{d} v\right] .
\end{aligned}
$$

Integrating by parts, we have that for any $f, h$

$$
\begin{aligned}
& \left(\int_{v_{0}^{-}}^{v_{0}^{+}} f(v) \mathrm{d} v\right) \int_{0}^{v_{0}^{+}} \frac{h(v)}{f(v)} \mathrm{d} v-\int_{v_{0}^{-}}^{v_{0}^{+}} f(v)\left(\int_{0}^{v} \frac{h(w)}{f(w)} \mathrm{d} w\right) \mathrm{d} v=\int_{v_{0}^{-}}^{v_{0}^{+}} \frac{h(v)}{f(v)}\left(\int_{v_{0}^{-}}^{v} f(w) \mathrm{d} w\right) \mathrm{d} v \\
& \left(\int_{v_{0}^{-}}^{v_{0}^{+}} f(v) \mathrm{d} v\right) \int_{0}^{v_{0}^{-}} \frac{h(v)}{f(v)} \mathrm{d} v-\int_{v_{0}^{-}}^{v_{0}^{+}} f(v)\left(\int_{0}^{v} \frac{h(w)}{f(w)} \mathrm{d} w\right) \mathrm{d} v=\int_{v_{0}^{-}}^{v_{0}^{+}} \frac{h(v)}{f(v)}\left(\int_{v}^{v_{0}^{+}} f(w) \mathrm{d} w\right) \mathrm{d} v .
\end{aligned}
$$

Using these identities for $f=\partial_{\mu} \varphi\left(\cdot, \mu_{0}\right)$ and $h=\partial_{\varepsilon}\left(\frac{\tilde{b}}{\tilde{a}}\right)\left(\varphi\left(\cdot, \mu_{0}\right), 0\right)$ we obtain that

$$
\begin{aligned}
\left(u^{+}\right)^{\prime}(0)= & \frac{\partial_{\mu} \varphi\left(v_{0}^{+}, \mu_{0}\right)}{\delta_{0} \int_{v_{0}^{-}}^{v_{0}^{+}} \partial_{\mu} \varphi\left(v, \mu_{0}\right) \mathrm{d} v}\left[\int_{v_{0}^{-}}^{v_{0}^{+}} \frac{\partial_{\varepsilon}\left(\frac{\tilde{b}}{\tilde{a}}\right)\left(\varphi\left(v, \mu_{0}\right), 0\right)}{\partial_{\mu} \varphi\left(v, \mu_{0}\right)}\left(\int_{v_{0}^{-}}^{v} \partial_{\mu} \varphi\left(w, \mu_{0}\right) \mathrm{d} w\right) \mathrm{d} v\right. \\
& \left.-\int_{v_{0}^{-}}^{v_{0}^{+}} \partial_{\varepsilon} \tilde{g}(v, 0) \mathrm{d} v\right]+\left(\frac{b}{a}\right)\left(\varphi\left(v_{0}^{+}, \mu_{0}\right)\right)\left(v^{+}\right)^{\prime}(0) ; \\
\left(u^{-}\right)^{\prime}(0)= & \frac{\partial_{\mu} \varphi\left(v_{0}^{-}, \mu_{0}\right)}{\delta_{0} \int_{v_{0}^{-}}^{v_{0}^{+}} \partial_{\mu} \varphi\left(v, \mu_{0}\right) \mathrm{d} v}\left[\int_{v_{0}^{-}}^{v_{0}^{+}} \frac{\partial_{\varepsilon}\left(\frac{\tilde{b}}{\tilde{a}}\right)\left(\varphi\left(v, \mu_{0}\right), 0\right)}{\partial_{\mu} \varphi\left(v, \mu_{0}\right)}\left(\int_{v}^{v_{0}^{+}} \partial_{\mu} \varphi\left(w, \mu_{0}\right) \mathrm{d} w\right) \mathrm{d} v\right. \\
& \left.-\int_{v_{0}^{-}}^{v_{0}^{+}} \partial_{\varepsilon} \tilde{g}(v, 0) \mathrm{d} v\right]+\left(\frac{b}{a}\right)\left(\varphi\left(v_{0}^{-}, \mu_{0}\right)\right)\left(v^{-}\right)^{\prime}(0) .
\end{aligned}
$$

To finish the proof of lemma, we subtract (2.21) from (2.20).

In the lemma below we compute the leading order term in the expansion of $u^{+}(\varepsilon)-u^{-}(\varepsilon)$. 
Lemma 2.5. The following assertions are true:

(i) If $u_{0}^{+} \neq u_{0}^{-}$then

$$
u^{+}(\varepsilon)-u^{-}(\varepsilon)=\left(u_{0}^{+}-u_{0}^{-}\right)+\mathcal{O}(\varepsilon)
$$

(ii) If $u_{0}^{+}=u_{0}^{-}=u^{\infty}$ and $c^{\infty}=\frac{b^{\prime}\left(u^{\infty}\right)}{a\left(u^{\infty}\right)}$ then

$$
u^{+}(\varepsilon)-u^{-}(\varepsilon)=K \varepsilon+\mathcal{O}\left(\varepsilon^{2}\right)
$$

where

$$
K=\left\{\begin{array}{l}
\frac{e^{c^{\infty} v_{0}^{+}-e^{c^{\infty}} v_{0}^{-}}}{c^{\infty} \delta_{0}} \partial_{\varepsilon}(\tilde{\tilde{b}})\left(u^{\infty}, 0\right)-\frac{c^{\infty}}{\delta_{0}} \int_{v_{0}^{-}}^{v_{0}^{+}} \partial_{\varepsilon} \tilde{g}(v, 0) \mathrm{d} v \text { if } c^{\infty} \neq 0 \\
\frac{v_{0}^{+}-v_{0}^{-}}{\delta_{0}} \partial_{\varepsilon}\left(\frac{\tilde{b}}{\tilde{a}}\right)\left(u^{\infty}, 0\right) \text { if } c^{\infty}=0 .
\end{array}\right.
$$

Proof. Assertion (i) follows from the smoothness of $u^{ \pm}(\cdot)$. To prove (ii), we note that since $\varphi\left(\cdot, \mu_{0}\right)$ is the solution of the autonomous differential equation $u^{\prime}=\frac{b(u)}{a(u)}$, it is monotone or constant. Using that $u_{0}^{+}=u_{0}^{-}=u^{\infty}$ we conclude that $b\left(u^{\infty}\right)=0$ and $\varphi\left(v, \mu_{0}\right)=u^{\infty}$ for all $v \in \mathbb{R}$. From Lemma 2.1(i) we obtain that

$$
\partial_{\mu} \varphi\left(v, \mu_{0}\right)=e^{c^{\infty} v} \quad \text { for all } \quad v \in \mathbb{R} .
$$

The formula for $K$ follows shortly from Lemma 2.4(iii).

In the second part of this section we study the robustness of layers under $\delta$-perturbations from $\delta_{0}=0$. In this case the properties of $H$, Lemma 2.2, are equivalent to

$$
g\left(v_{0}^{ \pm}\right)=0, \quad g^{\prime}\left(v_{0}^{ \pm}\right)<0, \quad \int_{v_{0}^{-}}^{v_{0}^{+}} g(v) \mathrm{d} v=0 .
$$

We define $\bar{H}: \mathbb{R}^{3} \rightarrow \mathbb{R}$ by

$$
\bar{H}(v, \mu, \delta)=\delta \varphi(v, \mu)+g(v) .
$$

To prove the $\delta$-robustness of layers we need to show that there exist smooth functions $\mu(\delta)$ and $v^{ \pm}(\delta)$ such that we have

(1) $\delta$-robustness of the equilibria $v_{0}^{ \pm}$:

(a) $v^{ \pm}(0)=v_{0}^{ \pm}$

(b) locally, the equation $\bar{H}(v, \mu(\delta), \delta)=0$ has the unique solution $v=v^{ \pm}(\delta)$;

(2) $\delta$-robustness of the equal area condition: $\int_{v^{-}(\delta)}^{v^{+}(\delta)} \bar{H}(v, \mu(\delta), \delta) \mathrm{d} v=0$.

In the next lemma we give a condition that is necessary and sufficient for the $\delta$-robustness of layers.

Lemma 2.6. Assume that (2.7) has a layer for $\delta_{0}=0$. Then, the layer is $\delta$-robust if and only if $\int_{\mathbb{R}} u^{*} v_{x}^{*}=0$. 
Proof. First, we remark that by making the change of variables $v=v^{*}$, we have that $\int_{\mathbb{R}} u^{*} v_{x}^{*}=\int_{v_{0}^{-}}^{v_{0}^{+}} \varphi\left(v, \mu_{0}\right) \mathrm{d} v$. Assume that $\int_{\mathbb{R}} u^{*} v_{x}^{*}=0$. Since

$$
\bar{H}\left(v_{0}^{ \pm}, \mu, 0\right)=g\left(v_{0}^{ \pm}\right)=0, \quad \partial_{v} \bar{H}\left(v_{0}^{ \pm}, \mu, 0\right)=g^{\prime}\left(v_{0}^{ \pm}\right)<0, \quad \text { for all } \quad \mu \in \mathbb{R},
$$

we conclude from the Implicit Functions Theorem that there exist two smooth functions $\bar{v}^{ \pm}(\mu, \delta)$

(i) $\bar{v}^{ \pm}(\mu, 0)=v_{0}^{ \pm}$;

(ii) For $\delta$ in a neighborhood of 0 , the equation $\bar{H}(v, \mu, \delta)=0$ has the unique solution $v=$ $\bar{v}^{ \pm}(\mu, \delta)$.

To prove the area preserving condition we have to show that the equation $A(\mu, \delta)=0$ can be solved for $\mu$ for any $\delta$ in a neighborhood of 0 . Here $A$ is defined by

$$
A(\mu, \delta)=\int_{\bar{v}^{-}(\mu, \delta)}^{\bar{v}^{+}(\mu, \delta)} \bar{H}(v, \mu, \delta) \mathrm{d} v .
$$

Next, we define the functions $M$ and $N$ through

$$
M(\mu, \delta)=\int_{\bar{v}^{-}(\mu, \delta)}^{\bar{v}^{+}(\mu, \delta)} \varphi(v, \mu) \mathrm{d} v, \quad N(\mu, \delta)=\int_{\bar{v}^{-}(\mu, \delta)}^{\bar{v}^{+}(\mu, \delta)} g(v) \mathrm{d} v .
$$

We note that $A(\mu, \delta)=\delta M(\mu, \delta)+N(\mu, \delta)$. Moreover, from (i), (ii) and (2.23) we have that

$$
\begin{aligned}
N(\mu, 0) & =\int_{\bar{v}^{-}(\mu, 0)}^{\bar{v}^{+}(\mu, 0)} g(v) \mathrm{d} v=\int_{v_{0}^{-}}^{v_{0}^{+}} g(v) \mathrm{d} v=0 ; \\
\partial_{\delta} N(\mu, 0) & =g\left(\bar{v}^{+}(\mu, 0)\right) \partial_{\delta} \bar{v}^{+}(\mu, 0)-g\left(\bar{v}^{-}(\mu, 0)\right) \partial_{\delta} \bar{v}^{-}(\mu, 0) \\
& =g\left(v_{0}^{+}\right) \partial_{\delta} \bar{v}^{+}(\mu, 0)-g\left(v_{0}^{-}\right) \partial_{\delta} \bar{v}^{-}(\mu, 0)=0,
\end{aligned}
$$

for all $\mu \in \mathbb{R}$, which implies that there is a smooth function $N_{1}(\cdot, \cdot)$ such that

$$
N(\mu, \delta)=\delta^{2} N_{1}(\mu, \delta)
$$

It follows that the equation $A(\mu, \delta)=0$ is equivalent to the equation

$$
M(\mu, \delta)+\delta N_{1}(\mu, \delta)=0 .
$$

We denote by $A_{1}(\mu, \delta)=M(\mu, \delta)+\delta N_{1}(\mu, \delta)$ the left hand side of the above equation. We compute

$$
\begin{aligned}
& A_{1}\left(\mu_{0}, 0\right)=M\left(\mu_{0}, 0\right)=\int_{\bar{v}^{-}\left(\mu_{0}, 0\right)}^{\bar{v}^{+}(\mu, 0)} \varphi(v, \mu) \mathrm{d} v=\int_{v_{0}^{-}}^{v_{0}^{+}} \varphi(v, \mu) \mathrm{d} v=0 \\
& \partial_{\mu} A_{1}(\mu, 0)=\partial_{\mu} M(\mu, 0)=\int_{\bar{v}^{-}(\mu, 0)}^{\bar{v}^{+}(\mu, 0)} \partial_{\mu} \varphi(v, \mu) \mathrm{d} v=\int_{v_{0}^{-}}^{v_{0}^{+}} \partial_{\mu} \varphi(v, \mu) \mathrm{d} v>0 .
\end{aligned}
$$

From the Implicit Function Theorem we have that there is a smooth function $\mu(\delta)$ such that 
(iii) $\mu(0)=\mu_{0}$;

(iv) For $\delta$ in a neighborhood of 0 , the equation $A(\mu, \delta)=0$ has the unique solution $\mu=\mu(\delta)$.

Define $v^{ \pm}(\delta)=\bar{v}^{ \pm}(\mu(\delta), \delta)$. From (ii) and (iv) we have that for $\delta$ in a neighborhood of 0 the equation $\bar{H}(v, \mu(\delta), \delta)=0$ has a unique solution $v=v^{ \pm}(\delta)$. Moreover,

$$
\int_{v^{-}(\delta)}^{v^{+}(\delta)} \bar{H}(v, \mu(\delta), \delta) \mathrm{d} v=\delta A_{1}(\mu(\delta), \delta)=0,
$$

proving the $\delta$-robustness of the layer.

To see that the condition $\int_{\mathbb{R}} u^{*} v_{x}^{*}=0$ is necessary, assume the $\delta$-robustness of the "equal area condition". That is, we have that there exists a smooth function $\bar{\mu}(\cdot)$ such that $\bar{\mu}(0)=\mu_{0}$ and

$$
M(\bar{\mu}(\delta), \delta)+\delta N(\bar{\mu}(\delta), \delta)=0
$$

for all $\delta$ in a neighborhood of 0 . It follows that $\int_{\mathbb{R}} u^{*} v_{x}^{*}=\int_{v_{0}^{-}}^{v_{0}^{+}} \varphi\left(v, \mu_{0}\right) \mathrm{d} v=M(\bar{\mu}(0), 0)=0$, proving the lemma.

\section{Essential spectrum}

In this section we compute the essential spectrum of the linearized operator $\mathcal{L}$ defined in (1.7) and study its stability. Throughout this section, we assume that $\left(u^{*}, v^{*}\right)$ is a layer of (1.1) for $\delta=\delta_{0}$, satisfying (1.8). Since $u^{*}$ and $v^{*}$ converge exponentially as $x \rightarrow \pm \infty$, we obtain that $v_{x}^{*} \rightarrow 0$ exponentially as $x \rightarrow \pm \infty$, by standard ODE arguments. Since $u_{x}^{*}=\frac{b^{*}}{a^{*}} v_{x}^{*}$, it follows that $u_{x}^{*} \rightarrow 0$ exponentially as $x \rightarrow \pm \infty$. We start this section with the following observation.

Remark 3.1. The following formula holds true:

$$
\mathcal{L}=D(x) \partial_{x}^{2}+M(x) \partial_{x}+N(x)
$$

where the matrix-valued functions $D, M$ and $N$ are continuous and have limits at $\pm \infty$, denoted $D^{ \pm}, M^{ \pm}$and $N^{ \pm}$. Moreover, $D$ is invertible with bounded inverse and

$$
D^{ \pm}=\left[\begin{array}{cc}
a^{ \pm} & -b^{ \pm} \\
0 & 1
\end{array}\right], \quad M^{ \pm}=0_{2}, \quad N^{ \pm}=\left[\begin{array}{cc}
0 & 0 \\
\delta_{0} & g^{\prime}\left(v^{ \pm}\right)
\end{array}\right]
$$

We recall the definition of the essential spectrum: we say that $\lambda$ belongs to the essential spectrum $\sigma_{\text {ess }}(\mathcal{L})$ if $\mathcal{L}-\lambda$ is not a Fredholm operator with index zero. We refer to the complement of the essential spectrum in the spectrum as the point spectrum, denoted $\sigma_{\text {point }}(\mathcal{L})$.

Lemma 3.2. The essential spectrum, the point spectrum of $\mathcal{L}$ and multiplicities are independent of the choice of function space, $X=L^{2}\left(\mathbb{R}, \mathbb{C}^{2}\right)$ and $X=B U C\left(\mathbb{R}, \mathbb{C}^{2}\right)$. Moreover, $\lambda \in \sigma_{\mathrm{ess}}(\mathcal{L})$ if and only if there exists $\tau \in \mathbb{R}$ and $\alpha \in\{+,-\}$ such that $\operatorname{det}\left(D^{\alpha} \tau^{2}+N^{\alpha}-\lambda I_{2}\right)=0$. 
Proof. To see that the essential spectrum of the operator $\mathcal{L}$ is independent of the choice of the function space, one can use arguments similar to the ones given in [9, Chapter, Appendix]. On the other hand, the point spectrum and the multiplicities of the eigenvalues are independent of the choice of function space, since the eigenvectors and the generalized eigenvectors decay exponentially at $\pm \infty$.

Since the matrix-valued function $D(\cdot)$ is continuous, bounded and $D^{-1}(\cdot)$ is bounded, we have that the operator of multiplication by $D(\cdot)$, denoted $M_{D}$, is invertible. It follows that $\mathcal{L}-\lambda$ is Fredholm with index 0 if and only if $M_{D^{-1}}(\mathcal{L}-\lambda)$ is Fredholm with index 0 . Since $M(x) \rightarrow 0$ exponentially as $x \rightarrow \pm \infty$, we infer that $M_{D^{-1}}(\mathcal{L}-\lambda)$ is a relatively compact perturbation of $\partial_{x}^{2}+D^{-1}(x)(N(x)-\lambda)$. Thus, $\lambda \in \sigma_{\text {ess }}(\mathcal{L})$ if and only if $\partial_{x}^{2}+D^{-1}(x)(N(x)-\lambda)$ is not

Fredholm with index 0 . The essential spectrum of the later can be computed using [9, Chapter, Theorem A2]. We conclude that $\lambda \in \sigma_{\text {ess }}(\mathcal{L})$ if and only if there exists $\tau \in \mathbb{R}$ such that $\operatorname{det}\left(\tau^{2} I_{2}+\left(D^{+}\right)^{-1}\left(N^{+}-\lambda\right)\right)=0$ or $\operatorname{det}\left(\tau^{2}+\left(D^{-}\right)^{-1}\left(N^{-}-\lambda\right)\right)=0$, proving the lemma.

As a consequence of this lemma we have

Remark 3.3. The essential spectrum consists of the image of four functions:

$$
\begin{gathered}
\sigma_{\text {ess }}(\mathcal{L})=\left\{\lambda_{j}^{ \pm}(\tau): \tau \in \mathbb{R}, j=1,2\right\}, \quad \text { where } \\
\lambda_{1}^{ \pm}(\tau)=\frac{\operatorname{tr}^{ \pm}(\tau)-\sqrt{\operatorname{tr}^{ \pm}(\tau)^{2}-4 \operatorname{det}^{ \pm}(\tau)}}{2}, \lambda_{2}^{ \pm}(\tau)=\frac{\operatorname{tr}^{ \pm}(\tau)+\sqrt{\operatorname{tr}^{ \pm}(\tau)^{2}-4 \operatorname{det}^{ \pm}(\tau)}}{2} \\
\operatorname{tr}^{ \pm}(\tau)=-\left(a^{ \pm}+1\right) \tau^{2}+g^{\prime}\left(v^{ \pm}\right), \operatorname{det}^{ \pm}(\tau)=a^{ \pm} \tau^{4}-\left(\delta_{0} b^{ \pm}+g^{\prime}\left(v^{ \pm}\right) a^{ \pm}\right) \tau^{2} .
\end{gathered}
$$

We conclude this section with:

Lemma 3.4. Under the assumptions of Theorem 1.1, the following assertions are true:

(i) $0 \in \sigma_{\text {ess }}(\mathcal{L})$;

(ii) $\sup \operatorname{Re} \sigma_{\text {ess }}(\mathcal{L}) \leq 0$ if and only if $g^{\prime}\left(v^{ \pm}\right) \leq 0$;

Proof. To prove (i) we note that $\operatorname{det}^{ \pm}(0)=0$, and thus, we have that $0=\lambda_{1}^{ \pm}(0) \in \sigma_{\text {ess }}(\mathcal{L})$. From the properties of $H$, Lemma 2.2 , it follows that $\operatorname{det}^{ \pm}(\tau) \geq 0$ for all $\tau \in \mathbb{R}$. Thus, $\sup \operatorname{Re} \sigma_{\text {ess }}(\mathcal{L}) \leq 0$ if and only if $\operatorname{tr}^{ \pm}(\tau) \leq 0$ for all $\tau \in \mathbb{R}$, proving (ii).

\section{Tracing the point spectrum: Preliminaries}

In this section we collect a few results needed in the later sections, concerning the kernels of $\mathcal{L}$ and its adjoint $\mathcal{L}^{*}$, the Fredholm properties of the operator $\mathcal{L}$ on weighted spaces and other related topics. Throughout this section we assume the hypotheses of Theorem 1.1, that is, we assume that (1.5) has an exponentially localized layer solution $\left(u^{*}, v^{*}\right)$ at $\delta=\delta_{0}$ and that the essential spectrum of the operator $\mathcal{L}$ is stable. As shown in Section 2, the equation (2.7) has a layer solution at $\delta=\delta_{0}$ for some fixed $\mu=\mu_{0}$ and $u^{*}=\varphi\left(v^{*}, \mu_{0}\right)$. 


\subsection{Homotopy}

In this subsection we construct a homotopy to an easier system, whose point spectrum can be studied easily. As a bonus, this homotopy will preserve the structure of the layer and the stability properties of the essential spectrum of $\mathcal{L}$. In what follows we consider $\delta$ between 0 and $\delta_{0}$ as a homotopy parameter and the system

$$
\left\{\begin{array}{l}
u_{t}=\left[a(u) u_{x}-b(u) v_{x}\right]_{x}, \\
v_{t}=v_{x x}+\delta u+\bar{g}(v, \delta)
\end{array}\right.
$$

where $\bar{g}: \mathbb{R}^{2} \rightarrow \mathbb{R}$ is defined by $\bar{g}(v, \delta)=g(v)+\left(\delta_{0}-\delta\right) \varphi\left(v, \mu_{0}\right)$. Since $g$ is a $C^{3}$ function and $\varphi\left(\cdot, \mu_{0}\right)$ is the solution of the Cauchy Problem $(2.3)$, we infer that $\bar{g}$ is a $C^{3}$ function.

Remark 4.1. The homotopy described in (4.1) satisfies the properties:

(i) One of the endpoints of the homotopy is the original system (1.1), since $\bar{g}\left(v, \delta_{0}\right)=g(v)$ for all $v \in \mathbb{R}$.

(ii) $\left(u^{*}, v^{*}\right)$ is a layer solution of (4.1) for all $\delta$, since $\bar{g}\left(v^{*}, \delta\right)+\delta u^{*}=g\left(v^{*}\right)+\delta_{0} u^{*}$ is independent of $\delta$ during the homotopy.

The linearization of (4.1) along the layer $\left(u^{*}, v^{*}\right)$ on $X \times X$, where $X$ is a function space, is given by

$$
\frac{\mathrm{d}}{\mathrm{d} t}\left(\begin{array}{l}
u \\
v
\end{array}\right)=\mathcal{L}(\delta)\left(\begin{array}{l}
u \\
v
\end{array}\right),
$$

where $\mathcal{L}(\delta)$ is defined by replacing in the definition $(1.7)$ of $\mathcal{L}, g^{\prime}\left(v^{*}\right)$ by $\partial_{v} \bar{g}\left(v^{*}, \delta\right)$ and $\delta_{0}$ by $\delta$. In the next lemma we show that the stability properties of the essential spectrum do not change during the homotopy.

Lemma 4.2. Assume the hypothesis of Theorem 1.1. Choosing the function space $X=$ $L^{2}\left(\mathbb{R}, \mathbb{C}^{2}\right)$ or $X=B U C\left(\mathbb{R}, \mathbb{C}^{2}\right)$, the essential spectrum of $\mathcal{L}(\delta)$ is stable for all $\delta$ between 0 and $\delta_{0}$.

Proof. We note that to prove the lemma, it is enough to prove that $\partial_{v} \bar{g}\left(v^{ \pm}, \delta\right)<0$ for all $\delta$ between 0 and $\delta_{0}$. Since $\varphi\left(\cdot, \mu_{0}\right)$ is a solution of $(2.3)$ we have that

$$
\partial_{v} \bar{g}(v, \delta)=g^{\prime}(v)+\left(\delta_{0}-\delta\right) \frac{b\left(\varphi\left(v, \mu_{0}\right)\right)}{a\left(\varphi\left(v, \mu_{0}\right)\right)},
$$

which implies that

$$
\partial_{v} \bar{g}\left(v^{ \pm}, \delta\right)=g^{\prime}\left(v^{ \pm}\right)+\left(\delta_{0}-\delta\right) \frac{b^{ \pm}}{a^{ \pm}} .
$$

We denote the right-hand side of this equation by $p^{ \pm}(\delta)$ and we note that $p^{ \pm}(\cdot)$ is a monotone function. Moreover, $p^{ \pm}\left(\delta_{0}\right)=g^{\prime}\left(v^{ \pm}\right)<0$ by assumption and $p^{ \pm}(0)=g^{\prime}\left(v^{ \pm}\right)+\delta_{0} \frac{b^{ \pm}}{a^{ \pm}}=$ $\partial_{v} H\left(v^{ \pm}, \mu_{0}\right)<0$ by the hyperbolicity property in Lemma 2.2. It follows that $p^{ \pm}(\delta)<0$ for all $\delta$ between 0 and $\delta_{0}$, proving the lemma. 


\subsection{Layer stability at $\delta=0$}

In this subsection we prove that for $\delta=0(4.2)$ has no unstable eigenvalue. A key feature at $\delta=0$ is the upper-triangular structure of the linearization $\mathcal{L}(0)$.

Lemma 4.3. Under the assumptions of Theorem 1.1, the layers in the system (4.1) for $\delta=0$ are stable, that is, $\mathcal{L}(0)$ has no eigenvalue with positive real part.

Proof. First, we note that if $\delta=0$ the operator $\mathcal{L}(0)$ has upper-triangular block structure

$$
\mathcal{L}(0)=\left[\begin{array}{cc}
\mathcal{K}_{1} & -\partial_{x}\left(b\left(u^{*}\right) \partial_{x}\right) \\
0 & \mathcal{K}_{2}
\end{array}\right],
$$

where $\mathcal{K}_{1}=\partial_{x}\left(a\left(u^{*}\right) \partial_{x}+a^{\prime}\left(u^{*}\right) u_{x}^{*}-b^{\prime}\left(u^{*}\right) v_{x}^{*}\right)$ and $\mathcal{K}_{2}=\partial_{x}^{2}+\partial_{v} \bar{g}\left(v^{*}, 0\right)$. This upper-triangular structure allows us to decouple the eigenvalue problem $\mathcal{L}\left(\begin{array}{l}u \\ v\end{array}\right)=\lambda\left(\begin{array}{l}u \\ v\end{array}\right)$. Since the operator $\mathcal{K}_{2}$ is Sturm-Liouvile and its kernel $v_{x}^{*}$ has no sign change because $v^{*}$ is monotone, we infer that $\mathcal{K}_{2}$ has no eigenvalue with positive real part. It follows that $\operatorname{Re} \lambda \leq 0$ or $v=0$. If $v=0$ then the $u$-equation of the eigenvalue problem for $\mathcal{L}(0)$ is equivalent to the eigenvalue problem $\mathcal{K}_{1} u=\lambda u$. Since the operator $\mathcal{K}_{1}$ is in divergence form, we have that $\mathcal{K}_{1}$ has no eigenvalue with positive real part. Indeed, any eigenvalue with positive real part would give rise to an exponentially growing solution of $u_{t}=\mathcal{K}_{1} u$ that is also exponentially localized in space, so that $\|u(t)\|_{L^{1}}$ would be growing exponentially. On the other hand, the semigroup generated by $\mathcal{K}_{1}$ is a contraction in $L^{1}$, as can readily be seen by using the fact that $\int u$ is conserved, splitting initial conditions into positive and negative parts, and exploiting positivity of the solution. This shows that there are no eigenvalues with positive real part and concludes the proof of the lemma.

Using the same Sturm-Liouvile theory arguments one can show that the same result is true in the case when $b \equiv 0$.

Lemma 4.4. Under the assumptions of Theorem 1.1, the layers in the system (1.1) for $b\left(u^{*}\right)=$ 0 are stable, that is, $\mathcal{L}$ has no eigenvalue with positive real part.

\subsection{The kernels of $\mathcal{L}$ and $\mathcal{L}^{*}$}

We note that the functions in the kernels of $\mathcal{L}$ and $\mathcal{L}^{*}$ are smooth functions for any choice of function space considered here. Thus, we begin by investigating the solutions of the ODE system

$$
\begin{gathered}
a^{*} u_{x}+\left(a^{\prime}\left(u^{*}\right) u_{x}^{*}-b^{\prime}\left(u^{*}\right) v_{x}^{*}\right) u-b^{*} v_{x}=0 \\
v_{x x}+\delta_{0} u+g^{\prime}\left(v^{*}\right) v=0 .
\end{gathered}
$$

The kernel of $\mathcal{L}$ can be described as follows.

Lemma 4.5. Under the assumptions of Theorem 1.1, the following assertions are true: 
(i) If $\delta_{0} \neq 0$ the kernel of $\mathcal{L}$ in $B U C\left(\mathbb{R}, \mathbb{C}^{2}\right)$ is spanned by $\left(u_{x}^{*}, v_{x}^{*}\right)^{\mathrm{T}}$;

(ii) If $\delta_{0}=0$ the kernel of $\mathcal{L}$ in $B U C\left(\mathbb{R}, \mathbb{C}^{2}\right)$ is spanned by $\left(u_{x}^{*}, v_{x}^{*}\right)^{\mathrm{T}}$ and $\left(\partial_{\mu} \varphi\left(v^{*}, \mu_{0}\right), 0\right)^{\mathrm{T}}$.

Proof. First, we note that for any $v \in B U C^{1}(\mathbb{R}), u=\frac{b^{*}}{a^{*}} v$ is a solution of (4.3). Thus, we can solve (4.3) for $u$ in terms of $v$ as follows:

$$
u=w+\frac{b^{*}}{a^{*}} v
$$

where $w$ is a solution of the equation

$$
a^{*} w_{x}+\left(a^{\prime}\left(u^{*}\right) u_{x}^{*}-b^{\prime}\left(u^{*}\right) v_{x}^{*}\right) w=0 .
$$

Dividing this equation by $a^{*} \geq a_{0}>0$ and using the fact that $u_{x}^{*}=\frac{b^{*}}{a^{*}} v_{x}^{*}$, we conclude that $w$ satisfies the equation

$$
w_{x}=\left[\left(\frac{b}{a}\right)^{\prime}\left(u^{*}\right) v_{x}^{*}\right] w .
$$

Since $u^{*}=\varphi\left(v^{*}, \mu_{0}\right)$ and $\partial_{\mu} \varphi\left(\cdot, \mu_{0}\right) \neq 0$ is a solution of the Cauchy Problem (2.4), we infer that $w=c \partial_{\mu} \varphi\left(v^{*}, \mu_{0}\right)$ for some complex constant $c$. It follows that

$$
u=c \partial_{\mu} \varphi\left(v^{*}, \mu_{0}\right)+\frac{b^{*}}{a^{*}} v .
$$

Substituting into (4.4) we obtain that $v$ satisfies the equation

$$
v_{x x}+\partial_{v} H\left(v^{*}, \mu_{0}\right) v=-c \delta_{0} \partial_{\mu} \varphi\left(v^{*}, \mu_{0}\right) .
$$

Next, we note that the equation

$$
v_{x x}+\partial_{v} H\left(v^{*}, \mu_{0}\right) v=0
$$

is the variational equation of $v_{x x}+H\left(v, \mu_{0}\right)=0$. Thus, all bounded solutions of (4.10) are of the form $v=\beta v_{x}^{*}$ for some complex constant $\beta$. Moreover, since $v_{x}^{*}$ is exponentially decaying at $\pm \infty$ we have that equation (4.9) has a bounded solution on $\mathbb{R}$ if and only if

$$
\int_{\mathbb{R}}\left(c \delta_{0} \partial_{\mu} \varphi\left(v^{*}, \mu_{0}\right)\right) v_{x}^{*}=0 .
$$

From Lemma 2.1(i) we know that $\partial_{\mu} \varphi\left(v^{*}, \mu_{0}\right)>0$ and since $v_{x}^{*}$ does not change sign, we conclude that the existence condition given in (4.11) is equivalent to $c \delta_{0}=0$. It follows that equation (4.9) is the same as equation (4.10) and thus $v=\beta v_{x}^{*}$.

Case $1, \delta_{0} \neq 0$ : In this case the solvability condition of equation (4.9) is equivalent to $c=0$. From (4.8) it follows that $u=\beta \frac{b^{*}}{a^{*}} v_{x}^{*}=\beta u_{x}^{*}$, proving that the kernel of $\mathcal{L}$ in $B U C\left(\mathbb{R}, \mathbb{C}^{2}\right)$ is spanned by $\left(u_{x}^{*}, v_{x}^{*}\right)^{\mathrm{T}}$.

Case 2, $\delta_{0}=0$ : From (4.8) it follows that $u=c \partial_{\mu} \varphi\left(v^{*}, \mu_{0}\right)+\beta \frac{b^{*}}{a^{*}} v_{x}^{*}=c \partial_{\mu} \varphi\left(v^{*}, \mu_{0}\right)+\beta u_{x}^{*}$, proving the lemma. 
We next address the kernel of the adjoint $\mathcal{L}^{*}$. Here and throughout, the adjoint is taken with respect to the $L^{2}$-pairing. Elements in the kernel then solve the ODE system

$$
\begin{gathered}
\left(a^{*} u_{x}\right)_{x}-\left(a^{\prime}\left(u^{*}\right) u_{x}^{*}-b^{\prime}\left(u^{*}\right) v_{x}^{*}\right) u_{x}+\delta_{0} v=0 ; \\
-\left(b^{*} u_{x}\right)_{x}+v_{x x}+g^{\prime}\left(v^{*}\right) v=0 .
\end{gathered}
$$

Next, we recall the definition of the Hilbert space $L_{\eta}^{2}\left(\mathbb{R}, \mathbb{C}^{2}\right)$ or, more generally $L^{2}(\mathbb{R}, \omega(x) \mathrm{d} x)$. The weighted space $L^{2}\left(\mathbb{R}, \mathbb{C}^{2}, \omega(x) \mathrm{d} x\right)$, with weight function $\omega(x)$ and norm

$$
\|u\|_{\omega}^{2}=\int_{\mathbb{R}}|u(x)|^{2} \omega(x) \mathrm{d} x
$$

is the space of all measurable functions with finite $\|\cdot\|_{\omega}$ norm. Here $L_{\eta}^{2}\left(\mathbb{R}, \mathbb{C}^{2}\right)$ is $L^{2}(\mathbb{R}, \omega(x) \mathrm{d} x)$ with $\omega(x)=e^{2 \eta|x|}$. Considering $\mathcal{L}$ as a densely defined, closed unbounded operator on $L_{\eta}^{2}\left(\mathbb{R}, \mathbb{C}^{2}\right)$, its adjoint $\mathcal{L}^{*}$ is a closed, densely defined unbounded operator on the dual of $L_{\eta}^{2}\left(\mathbb{R}, \mathbb{C}^{2}\right)$, which, using the $L^{2}$-pairing, can be identified with $L_{-\eta}^{2}\left(\mathbb{R}, \mathbb{C}^{2}\right)$.

Lemma 4.6. Under the assumptions of Theorem 1.1, the kernel of $\mathcal{L}^{*}$ in $L_{-\eta}^{2}\left(\mathbb{R}, \mathbb{C}^{2}\right), \eta>0$ sufficiently small, is spanned by $(1,0)^{\mathrm{T}},\left(\bar{U}_{1}, 0\right)^{\mathrm{T}}$ and $\left(\bar{U}_{2}, v_{x}^{*}\right)^{\mathrm{T}}$, where $\bar{U}_{1}^{\prime}=\frac{1}{a^{*} \partial_{\mu} \varphi\left(v^{*}, \mu_{0}\right)}, \bar{U}_{2}^{\prime}=$ $\frac{-\bar{M}}{a^{*} \partial_{\mu} \varphi\left(v^{*}, \mu_{0}\right)}$ and $\bar{M}^{\prime}=\delta_{0} \partial_{\mu} \varphi\left(v^{*}, \mu_{0}\right) v_{x}^{*}$.

Proof. Multiplying equation (4.12) by $\frac{b^{*}}{a^{*}}$ and then adding it to equation (4.13), we obtain the equation

$$
v_{x x}+\partial_{v} H\left(v^{*}, \mu_{0}\right) v=0,
$$

that is, $v$ satisfies equation (4.10). As shown in Lemma 4.5, it follows that $v=\beta v_{x}^{*}$ for some complex constant $\beta$. Using equation (4.12) it follows that

$$
\left(a^{*} u_{x}\right)_{x}-\left(a^{\prime}\left(u^{*}\right) u_{x}^{*}-b^{\prime}\left(u^{*}\right) v_{x}^{*}\right) u_{x}+\delta_{0} \beta v_{x}^{*}=0 .
$$

Making the change of variables $w=a^{*} u_{x}$ we obtain the equation

$$
w_{x}-\frac{a^{\prime}\left(u^{*}\right) u_{x}^{*}-b^{\prime}\left(u^{*}\right) v_{x}^{*}}{a^{*}} w+\delta_{0} \beta v_{x}^{*}=0 .
$$

Next, we note that, for $\beta=0$, the above equation is the adjoint equation of (4.7). Thus, we conclude that the solution of (4.14) is given by

$$
w=\alpha\left(\partial_{\mu} \varphi\left(v^{*}, \mu_{0}\right)\right)^{-1}-\beta\left(\partial_{\mu} \varphi\left(v^{*}, \mu_{0}\right)\right)^{-1} \bar{M},
$$

where $\alpha$ is a complex constant and $\bar{M}^{\prime}=\delta_{0} \partial_{\mu} \varphi\left(v^{*}, \mu_{0}\right) v_{x}^{*}$. We infer that $u=c+\alpha \bar{U}_{1}+\beta \bar{U}_{2}$, where $\bar{U}_{1}^{\prime}=\frac{1}{a^{*} \partial_{\mu} \varphi\left(v^{*}, \mu_{0}\right)}, \bar{U}_{2}^{\prime}=\frac{-\bar{M}}{a^{*} \partial_{\mu} \varphi\left(v^{*}, \mu_{0}\right)}$, proving the lemma.

Remark 4.7. The functions $\bar{U}_{1}$ and $\bar{U}_{2}$ have linear growth when $\delta_{0} \neq 0$. Moreover, if $\delta_{0}=0$, we can choose $\bar{M}=0$ and $\bar{U}_{2}=0$. In particular, the kernel of $\mathcal{L}^{*}$ in $B U C\left(\mathbb{R}, \mathbb{C}^{2}\right)$ is onedimensional whenever $\delta_{0} \neq 0$ and two-dimensional for $\delta_{0}=0$, the same dimensions that we computed for the kernel of $\mathcal{L}$, even though $\mathcal{L}$ is not Fredholm in $B U C\left(\mathbb{R}, \mathbb{C}^{2}\right)$. 


\subsection{The Fredholm properties on weighted spaces}

As we saw earlier, $0 \in \sigma_{\text {ess }}(\mathcal{L})$ when $\mathcal{L}$ is considered in $L^{2}$. This precludes a direct perturbation approach via Lyapunov-Schmidt reduction to the eigenvalue problem. In this section we prove that $\mathcal{L}$ is Fredholm on exponentially weighted spaces. This result is crucial for the later sections were we use Lyapunov-Schmidt reduction to construct a bifurcation function that tracks the eigenvalues of $\mathcal{L}$.

Lemma 4.8. There exists $\eta^{*}>0$ such that for all $\eta \in\left(0, \eta^{*}\right), \mathcal{L}$ is Fredholm on $L_{\eta}^{2}\left(\mathbb{R}, \mathbb{C}^{2}\right)$ with index $\operatorname{ind}(\mathcal{L})=-2$.

Proof. The proof follows closely [22]. The main idea is to show that we can transform the problem at hand to a Fredholm problem for a second order differential operator on $L^{2}\left(\mathbb{R}, \mathbb{C}^{2}\right)$ with no weight. Then, we construct a first order differential operator on $L^{2}\left(\mathbb{R}, \mathbb{C}^{4}\right)$ whose Fredholm properties coincide with those of $\mathcal{L}$. Finally, we use Palmer's results from [20, 21] to compute the Fredholm index.

Let $\eta>0$ and let $\psi \in C^{\infty}(\mathbb{R})$ be a smooth function satisfying the conditions: $\psi(x)=e^{-\eta|x|}$ for all $x \in \mathbb{R}$ with $|x| \geq 1$ and $\inf _{|x| \leq 1} \psi(x)>0$. One can easily check that $L_{\eta}^{2}\left(\mathbb{R}, \mathbb{C}^{2}\right)=$ $L^{2}\left(\mathbb{R}, \mathbb{C}^{2},[\psi(x)]^{-2} d x\right)$ with equivalent norms $\|\cdot\|_{L_{\eta}^{2}}$ and $\|\cdot\|_{L_{\psi^{-2}}^{2}}$.

Next, define the isomorphism $U_{\psi}: L^{2}\left(\mathbb{R}, \mathbb{C}^{2}\right) \rightarrow L_{\eta}^{2}(\mathbb{R}, \mathbb{C})$ by $U_{\psi} w=\psi w$. One readily verifies that $U_{\psi}$ is a bounded, invertible operator with bounded inverse. In consequence, the operator $\mathcal{L}$ is Fredholm on $L_{\eta}^{2}\left(\mathbb{R}, \mathbb{C}^{2}\right)$ if and only if $\mathcal{L}_{\psi}=U_{\psi}^{-1} \mathcal{L} U_{\psi}$ is Fredholm on $L^{2}\left(\mathbb{R}, \mathbb{C}^{2}\right)$ and their indices coincide.

From (3.1) we know that $\mathcal{L}=D(x) \partial_{x}^{2}+M(x) \partial_{x}+N(x)$, which implies that

$$
\mathcal{L}_{\psi}=D(x)\left(\partial_{x}+\frac{\psi^{\prime}(x)}{\psi(x)}\right)^{2}+M(x)\left(\partial_{x}+\frac{\psi^{\prime}(x)}{\psi(x)}\right)+N(x) .
$$

Next, we define the matrix-valued functions $A, A_{\psi}: \mathbb{R} \rightarrow \mathbb{C}^{4 \times 4}$ by

$$
\begin{gathered}
A(x)=\left[\begin{array}{cc}
0_{2} & I_{2} \\
-D(x)^{-1} N(x) & -D(x)^{-1} M(x)
\end{array}\right] \\
A_{\psi}(x)=\left[\begin{array}{cc}
0_{2} & I_{2} \\
-D(x)^{-1} N(x)-\frac{\psi^{\prime}(x)}{\psi(x)} D(x)^{-1} M(x)-\frac{\psi^{\prime \prime}(x)}{\psi(x)} & -D(x)^{-1} M(x)+2 \frac{\psi^{\prime}(x)}{\psi(x)}
\end{array}\right],
\end{gathered}
$$

where $0_{2}=\left[\begin{array}{ll}0 & 0 \\ 0 & 0\end{array}\right]$ and $I_{2}=\left[\begin{array}{ll}1 & 0 \\ 0 & 1\end{array}\right]$. From [26, Theorem A2] it follows that $\mathcal{L}_{\psi}$ is Fredholm on $L^{2}\left(\mathbb{R}, \mathbb{C}^{2}\right)$ if and only if $\mathcal{T}_{\psi}=\partial_{x}-A_{\psi}(x)$ is Fredholm on $L^{2}\left(\mathbb{R}, \mathbb{C}^{4}\right)$ and their indices coincide. Let $J_{\psi}: \mathbb{R} \rightarrow \mathbb{C}^{4 \times 4}$ be the matrix-valued function defined by

$$
J_{\psi}(x)=\left[\begin{array}{cc}
I_{2} & \left(1-\frac{\psi^{\prime}(x)}{\psi(x)}\right) I_{2} \\
0_{2} & I_{2}
\end{array}\right] .
$$

Since $J_{\psi}$ is continuous on $\mathbb{R}$ and $\operatorname{det} J_{\psi}(x)=1$ for all $x \in \mathbb{R}$, we infer that the operator of multiplication by $J_{\psi}$ is bounded, invertible with bounded inverse on $L^{2}\left(\mathbb{R}, \mathbb{C}^{4}\right)$. Using this 
transformation, one can see that $\mathcal{T}_{\psi}$ is conjugate to $\tilde{\mathcal{T}}_{\psi}=\partial_{x}+\frac{\psi^{\prime}(x)}{\psi(x)} I_{4}-A(x)$. Hence, $\mathcal{L}$ is Fredholm on $L_{\eta}^{2}\left(\mathbb{R}, \mathbb{C}^{2}\right)$ if and only if $\tilde{\mathcal{T}}_{\psi}$ is Fredholm on $L^{2}\left(\mathbb{R}, \mathbb{C}^{4}\right)$.

Using Remark 3.1, we obtain that

$$
A(x) \underset{|x| \rightarrow \infty}{\longrightarrow} A^{ \pm}, \quad \text { where } \quad A^{ \pm}=\left[\begin{array}{cc}
0_{2} & I_{2} \\
-\left(D^{ \pm}\right)^{-1} N^{ \pm} & 0_{2}
\end{array}\right] .
$$

Using Palmer's classical results, from $[20,21]$ we know that the operator $\tilde{\mathcal{T}}_{\psi}$ is Fredholm if and only if $A_{ \pm, \eta}:=\lim _{x \rightarrow \pm \infty}\left(A(x)-\frac{\psi^{\prime}(x)}{\psi(x)} I_{4}\right)$ are hyperbolic, and its Fredholm index is given by the difference of the Morse indices,

$$
\operatorname{ind}\left(\tilde{\mathcal{T}}_{\psi}\right)=i\left(A_{-, \eta}\right)-i\left(A_{+, \eta}\right)
$$

The eigenvalues of $A^{ \pm}$are $\pm \sqrt{-\frac{b^{ \pm}}{a^{ \pm}} \delta_{0}-g^{\prime}\left(v^{ \pm}\right)}= \pm \sqrt{-\partial_{v} H\left(v^{ \pm}, \mu_{0}\right)}$ with multiplicity 1 and 0 with multiplicity 2. Since $\lim _{x \rightarrow \pm \infty} \frac{\psi^{\prime}(x)}{\psi(x)}=\mp \eta$ we obtain that $A_{ \pm, \eta}=A^{ \pm} \pm \eta I_{4}$.

Define $\eta^{*}=\frac{1}{2} \min \left\{\sqrt{-\partial_{v} H\left(v^{+}, \mu_{0}\right)}, \sqrt{-\partial_{v} H\left(v^{-}, \mu_{0}\right)}\right\}>0$. We infer that $A_{ \pm, \eta}$ are hyperbolic, and the dimension of the unstable subspaces are the Morse indices $i\left(A_{-, \eta}\right)=1$ and $i\left(A_{+, \eta}\right)=3$ for all $\eta \in\left(0, \eta^{*}\right)$. This shows that $\tilde{\mathcal{T}}_{\psi}$ is Fredholm with index $\operatorname{ind}\left(\tilde{\mathcal{T}}_{\psi}\right)=-2$, proving the lemma.

\subsection{Asymptotics of eigenfunctions}

Let $\mathcal{L}^{ \pm}=D^{ \pm} \partial_{x}^{2}+N^{ \pm}$and consider the eigenvalue problems associated with $\mathcal{L}^{ \pm}$on $\mathbb{R}_{ \pm}$, respectively:

$$
\left\{\begin{array}{l}
a^{ \pm} u_{x x}+b^{ \pm} v_{x x}=\lambda u, x \in \mathbb{R}_{ \pm} \\
v_{x x}+\delta_{0} u+g^{\prime}\left(v^{ \pm}\right) v=\lambda v, x \in \mathbb{R}_{ \pm}
\end{array}\right.
$$

We define the linear dispersion relations by

$$
\Lambda^{ \pm}(\lambda, \nu)=\left[\begin{array}{cc}
a^{ \pm} \nu^{2}-\lambda & b^{ \pm} \nu^{2} \\
\delta_{0} & \nu^{2}+g^{\prime}\left(v^{ \pm}\right)-\lambda
\end{array}\right] \quad \text { and } \quad d^{ \pm}(\lambda, \nu)=\operatorname{det} \Lambda^{ \pm}(\lambda, \nu) .
$$

Lemma 4.9. The following assertions are true:

(i) If $\operatorname{Re} \lambda>0$ then equation $d^{ \pm}(\lambda, \nu)=0$ has two roots, $\nu_{ \pm}$and $\rho_{ \pm}$, with $\operatorname{Re} \nu_{ \pm}>0$ and $\operatorname{Re} \rho_{ \pm}>0$

(ii) Setting $\lambda=\gamma^{2}, \nu_{ \pm}$and $\rho_{ \pm}$are analytic in $\gamma$ on a neighborhood of 0 and

$$
\nu_{ \pm}(\gamma)=\sqrt{\frac{g^{\prime}\left(v^{ \pm}\right)}{a^{ \pm} g^{\prime}\left(v^{ \pm}\right)+\delta_{0} b^{ \pm}}} \gamma+\mathcal{O}\left(\gamma^{2}\right), \quad \rho_{ \pm}(\gamma)=\sqrt{-\partial_{v} H\left(v^{ \pm}, \mu_{0}\right)}+\mathcal{O}\left(\gamma^{2}\right) .
$$

Proof. (i) From Lemma 3.2 we have that

$$
\sigma_{\text {ess }}(\mathcal{L})=\left\{\lambda \in \mathbb{C}: \exists \tau \in \mathbb{R}, d^{+}(\lambda, i \tau)=0 \text { or } d^{-}(\lambda, i \tau)=0\right\} .
$$


and since in this section we assumed that $\operatorname{Re} \sigma_{\text {ess }}(\mathcal{L}) \leq 0$, we obtain that

$$
d^{ \pm}(\lambda, i \tau) \neq 0 \quad \text { for all } \quad \tau \in \mathbb{R}, \lambda \in \mathbb{C} \quad \text { with } \quad \operatorname{Re} \lambda>0 .
$$

Moreover, since $\Lambda^{ \pm}(\lambda, \nu)$ depends only on $\nu^{2}$ it follows that the equation $d^{ \pm}(\lambda, \nu)=0$ has exactly two roots $\nu_{ \pm}$and $\rho_{ \pm}$, with $\operatorname{Re} \nu_{ \pm}>0$ and $\operatorname{Re} \rho_{ \pm}>0$.

(ii) Equation $d^{ \pm}(0, \nu)=0$ has 0 as a double root and $\pm \sqrt{-\partial_{v} H\left(v^{ \pm}, \mu_{0}\right)}$ as simple roots. Expanding the simple roots in $\gamma^{2}$ we conclude $\rho_{ \pm}(\gamma)=\sqrt{-\partial_{v} H\left(v^{ \pm}, \mu_{0}\right)}+\mathcal{O}\left(\gamma^{2}\right)$. Expanding $d^{ \pm}\left(\gamma^{2}, \nu\right)$ in $\gamma^{2}$ we obtain that

$$
\nu^{2}\left(a^{ \pm} g^{\prime}\left(v^{ \pm}\right)+\delta_{0} b^{ \pm}\right)=g^{\prime}\left(v^{ \pm}\right) \gamma^{2}+\mathcal{O}\left(\gamma^{4}\right)+\mathcal{O}\left(\nu^{4}\right)
$$

At this point, the lemma follows shortly using Newton's polygon.

Lemma 4.10. Setting $\lambda=\gamma^{2}$, the following assertions are true:

(i) If $\operatorname{Re} \lambda>0$ the solutions of (4.16) bounded at $\pm \infty$ are of the form

$$
(u(x), v(x))^{\mathrm{T}}=c_{1} \alpha_{ \pm}(\gamma) e^{\mp \nu_{ \pm}(\gamma) x}+c_{2} \zeta_{ \pm}(\gamma) e^{\mp \rho_{ \pm}(\gamma) x}
$$

where $c_{1}, c_{2} \in \mathbb{C}$ and $\alpha_{ \pm}(\gamma), \zeta_{ \pm}(\gamma) \in \mathbb{C}^{2}$

(ii) The function $\alpha_{ \pm}$is analytic in $\gamma$ in a neighborhood of 0 . Moreover, $\alpha_{ \pm}$has the following expansion

$$
\alpha_{ \pm}(\gamma)=\left(-g^{\prime}\left(v^{ \pm}\right), \delta_{0}\right)^{\mathrm{T}}+\mathcal{O}\left(\gamma^{2}\right)
$$

Proof. Assertion (i) follows directly from Lemma 4.9(i). Using the expansion for $\nu_{ \pm}$and the ansatz $\alpha_{ \pm}(\gamma)=\left(-g^{\prime}\left(v^{ \pm}\right), \delta_{0}\right)^{\mathrm{T}}+\alpha_{2}^{ \pm}(\gamma)$ in the eigenvalue equation we obtain that

$$
\alpha_{2}^{ \pm}(\gamma)=\left(\gamma^{2}-\nu_{ \pm}(\gamma)^{2}, 0\right)^{\mathrm{T}}=\mathcal{O}\left(\gamma^{2}\right)
$$

proving the lemma.

Remark 4.11. Lemmas 4.5-4.10 are valid for $\mathcal{L}(\delta)$ for all $\delta$ between 0 and $\delta_{0}$ and the functions $\nu_{ \pm}$and $\alpha_{ \pm}$above can be extended to smooth functions on $\delta$, denoted $\hat{\nu}_{ \pm}$and $\hat{\alpha}_{ \pm}$. Moreover,

$\hat{\nu}_{ \pm}(\gamma, \delta)=\sqrt{\frac{g^{\prime}\left(v^{ \pm}\right)+\left(\delta_{0}-\delta\right) \frac{b^{ \pm}}{a^{ \pm}}}{a^{ \pm} g^{\prime}\left(v^{ \pm}\right)+\delta_{0} b^{ \pm}}} \gamma+\mathcal{O}\left(\gamma^{2}\right), \quad \hat{\alpha}_{ \pm}(\gamma, \delta)=\left(-g^{\prime}\left(v^{ \pm}\right)+\left(\delta-\delta_{0}\right) \frac{b^{ \pm}}{a^{ \pm}}, \delta\right)^{\mathrm{T}}+\mathcal{O}\left(\gamma^{2}\right)$.

This fact follows from the smoothness of eigenfunctions and eigenvectors of the asymptotic problem.

\section{Setting up the bifurcation problem}

In this section we investigate the eigenvalue problem for $\mathcal{L}$ in a neighborhood of 0 . We recast the eigenvalue problem on $L^{2}\left(\mathbb{R}, \mathbb{C}^{2}\right)$ as a generalized eigenvalue problem on exponentially 
weighted spaces $L_{\eta}^{2}\left(\mathbb{R}, \mathbb{C}^{2}\right)$ and show that the eigenvalue problem near $\lambda=0$ can be recast as a bifurcation problem that is analytic in $\sqrt{\lambda}$ and can be approached using Lyapunov-Schmidt reduction. Throughout this section we assume the hypotheses of Theorem 1.1, that is, we assume that (1.5) has an exponentially localized layer solution $\left(u^{*}, v^{*}\right)$ at $\delta=\delta_{0}$. As shown in Section 2, equation (2.7) then has a layer solution at $\delta=\delta_{0}$ for some fixed $\mu=\mu_{0}$ and $u^{*}=\varphi\left(v^{*}, \mu_{0}\right)$.

To solve the eigenvalue problem

$$
\left(\mathcal{L}-\gamma^{2}\right)\left(\begin{array}{l}
u \\
v
\end{array}\right)=0
$$

we use the ansatz

$$
(u(x), v(x))^{\mathrm{T}}=\underline{w}(x)+\beta_{1} \alpha_{+}(\gamma) \chi_{+}(x) e^{-\nu_{+}(\gamma) x}+\beta_{2} \alpha_{-}(\gamma) \chi_{-}(x) e^{\nu_{-}(\gamma) x} .
$$

where $\underline{w} \in L_{\eta}^{2}\left(\mathbb{R}, \mathbb{C}^{2}\right), \beta_{1}, \beta_{2} \in \mathbb{C}$, and $\nu_{ \pm}(\cdot), \alpha_{ \pm}(\cdot)$ are defined in Lemma 4.9 and Lemma 4.10, respectively. Here $\chi_{+}(x)=\frac{1+\rho(x)}{2}$, where $\rho \in C^{\infty}(\mathbb{R})$ is a smooth even function satisfying $\rho(x)=-1$ for all $x \leq-1, \rho(x)=1$ for all $x \geq 1$ and $\chi_{-}(x)=1-\chi_{+}(x)$.

Let $\varepsilon_{0}>0$ be small enough such that $\nu_{ \pm}$and $\alpha_{ \pm}$are analytic on $B\left(0, \varepsilon_{0}\right)$, the open complex ball centered at 0 and of radius $\varepsilon_{0}$, as shown in Lemma 4.9 and Lemma 4.10, respectively. For $\gamma \in B\left(0, \varepsilon_{0}\right)$ and $\eta \in\left(0, \eta^{*}\right)$, we define $\mathcal{T}(\gamma): H_{\eta}^{2}\left(\mathbb{R}, \mathbb{C}^{2}\right) \times \mathbb{C}^{2} \rightarrow L_{\eta}^{2}\left(\mathbb{R}, \mathbb{C}^{2}\right)$ by

$$
\mathcal{T}(\gamma)\left(\underline{w}, \beta_{1}, \beta_{2}\right)^{\mathrm{T}}=\left(\mathcal{L}-\gamma^{2}\right)\left[\underline{w}+\beta_{1} \alpha_{+}(\gamma) h_{+}(\gamma)+\beta_{2} \alpha_{-}(\gamma) h_{-}(\gamma)\right]
$$

Here, $\left[h_{ \pm}(\gamma)\right](x)=\chi_{ \pm}(x) e^{\mp \nu_{ \pm}(\gamma) x}$. In the next few lemmas we will prove that $\mathcal{T}$ is well defined and analytic on a neighborhood of 0 . First, we will show that $h_{ \pm}$are analytic in the following sense:

Lemma 5.1. Under the assumptions of Theorem 1.1, there is $\varepsilon_{0}$ small enough such that the functions $h_{ \pm}: B\left(0, \varepsilon_{0}\right) \rightarrow H_{-\eta}^{2}(\mathbb{R}, \mathbb{C})$ are analytic for $0<\eta<\eta^{*}$.

Proof. Since the functions $\nu_{ \pm}$and $\alpha_{ \pm}$are analytic on $B\left(0, \varepsilon_{0}\right)$ and $\nu_{ \pm}(0)=0$, it follows that we can choose $\varepsilon_{0}>0$ sufficiently small such that

$$
\left|\nu_{ \pm}(\gamma)\right| \leq \frac{\eta}{2} \quad \text { and } \quad\left|\alpha_{ \pm}(\gamma)\right| \leq M \quad \text { for all } \quad \gamma \in B\left(0, \varepsilon_{0}\right)
$$

where $M>\max \left\{\left|\alpha_{+}(0)\right|+1,\left|\alpha_{-}(0)\right|+1\right\}$. From these estimates we obtain that

$$
\left|\left[h_{ \pm}(\gamma)\right](x)\right| \leq\left|\alpha_{ \pm}(\gamma)\right| e^{\operatorname{Re} \nu_{ \pm}(\gamma)|x|} \leq M e^{\eta / 2|x|} \quad \text { for all } \quad \gamma \in B\left(0, \varepsilon_{0}\right), x \in \mathbb{R} .
$$

Using this last estimate, we infer that $h_{ \pm}(\gamma) \in L_{-\eta}^{2}(\mathbb{R}, \mathbb{C})$ for all $\gamma \in B\left(0, \varepsilon_{0}\right)$. To prove the lemma, we will prove first that $h_{ \pm}: B\left(0, \varepsilon_{0}\right) \rightarrow L_{-\eta}^{2}(\mathbb{R}, \mathbb{C})$ are analytic. Using again (5.5) together with Lebesgue's Dominated Convergence Theorem we have that $h_{ \pm}: B\left(0, \varepsilon_{0}\right) \rightarrow$ $L_{-\eta}^{2}(\mathbb{R}, \mathbb{C})$ are continuous. To show that $h_{ \pm}$are analytic it is enough to show that they are 
weakly analytic. To prove this last statement, for $f \in L_{\eta}^{2}(\mathbb{R}, \mathbb{C})$ let $h_{ \pm}^{f}=\left\langle h_{ \pm}(\cdot), f\right\rangle_{L^{2}}$ and let $R$ a rectangle contained in $B\left(0, \varepsilon_{0}\right)$. We integrate on the boundary of $\mathrm{R}$ using Fubini's Theorem:

$$
\oint_{\partial \mathrm{R}} h_{ \pm}^{f}(\gamma) \mathrm{d} \gamma=\int_{\mathbb{R}}\left(\oint_{\partial \mathrm{R}}\left(e^{\mp \nu_{ \pm}(\gamma) x}\right) \mathrm{d} \gamma\right) \overline{f(x)} d x=0
$$

This concludes the proof of analyticity of the maps $h_{ \pm}: B\left(0, \varepsilon_{0}\right) \rightarrow L_{-\eta}^{2}(\mathbb{R}, \mathbb{C})$. Furthermore, we note that $\partial_{x}\left(h_{ \pm}\right)$and $\partial_{x}^{2}\left(h_{ \pm}\right)$are linear combinations of products of $\chi_{ \pm}^{(k)}, \nu_{1}(\gamma)^{j}$ and $h_{ \pm}(\gamma)$, $k, j=0,1,2$, which concludes the proof of the lemma.

Lemma 5.2. Under the assumptions of Theorem 1.1, the map $\mathcal{T}(\cdot)$ is well-defined and analytic on $B\left(0, \varepsilon_{0}\right)$.

Proof. To prove the lemma it is enough to show that the maps $\mathcal{T}_{ \pm}: B\left(0, \varepsilon_{0}\right) \rightarrow L_{\eta}^{2}\left(\mathbb{R}, \mathbb{C}^{2}\right)$ defined by

$$
\mathcal{T}_{ \pm}(\gamma)=\left(\mathcal{L}-\gamma^{2}\right)\left(\alpha_{ \pm}(\gamma) h_{ \pm}(\gamma)\right)
$$

are well-defined and analytic. We start by introducing functions $D^{\infty}, N^{\infty}: \mathbb{R} \rightarrow \mathbb{C}^{2 \times 2}$ by

$$
D^{\infty}(x)=\left\{\begin{array}{l}
D^{+} \text {if } x \geq 0 \\
D^{-} \text {if } x<0
\end{array} \quad N^{\infty}(x)=\left\{\begin{array}{l}
N^{+} \text {if } x \geq 0 \\
N^{-} \text {if } x<0
\end{array}\right.\right.
$$

and $\mathcal{L}^{\infty}=D^{\infty}(x) \partial_{x}^{2}+N^{\infty}(x)$. Also, we consider $\psi \in C^{\infty}(\mathbb{R})$, such that $0 \leq \psi \leq 1, \psi(x)=1$ for all $x \in[-1,1]$ and $\psi(x)=0$ for all $x \in \mathbb{R}$ with $|x| \geq 2$. Since $\chi_{ \pm}(x)=0$ for $\pm x \geq 1$ and since from Lemma 4.10 we know that $\alpha_{ \pm}(\gamma) e^{\mp \nu_{ \pm}(\gamma) x}$ is a solution of equation (4.16), we have that

$$
\left[\left(\mathcal{L}^{\infty}-\gamma^{2}\right)\left(\alpha_{ \pm}(\gamma) h_{ \pm}(\gamma)\right)\right](x)=0 \quad \text { for all } \quad x \in \mathbb{R} \quad \text { with } \quad|x| \geq 1
$$

Moreover, since $\psi^{\prime}(x)=\psi^{\prime \prime}(x)=0$ for all $x \in[-1,1]$ we have that

$$
\chi_{[-1,1]} \mathcal{L}^{\infty}(\psi \underline{w})=\chi_{[-1,1]} \mathcal{L}^{\infty} \underline{w} \quad \text { for all } \quad \underline{w} \in H_{\mathrm{loc}}^{2}\left(\mathbb{R}, \mathbb{C}^{2}\right),
$$

where $\chi_{[-1,1]}$ is the characteristic function of the interval $[-1,1]$. From (5.7) and (5.8) we have that

$$
\left(\mathcal{L}^{\infty}-\gamma^{2}\right)\left(\alpha_{ \pm}(\gamma) h_{ \pm}(\gamma)\right)=\chi_{[-1,1]}\left(\mathcal{L}^{\infty}-\gamma^{2}\right)\left(\alpha_{ \pm}(\gamma) h_{ \pm}(\gamma)\right)=\chi_{[-1,1]}\left(\mathcal{L}^{\infty}-\gamma^{2}\right)\left(\psi \alpha_{ \pm}(\gamma) h_{ \pm}(\gamma)\right)
$$

for all $\gamma \in B\left(0, \varepsilon_{0}\right)$. Since $\mathcal{L}-\mathcal{L}^{\infty}$ is a second order differential operator with matrix-valued coefficients that decay exponentially at $\pm \infty$, it follows that

$$
\mathcal{L}-\mathcal{L}^{\infty} \quad \text { is bounded from } H_{-\eta}^{2}\left(\mathbb{R}, \mathbb{C}^{2}\right) \text { to } L_{\eta}^{2}\left(\mathbb{R}, \mathbb{C}^{2}\right)
$$

Next, we denote by $M_{\psi}$ the operator of multiplication by $\psi$. Since $\psi \in C^{\infty}(\mathbb{R})$ has compact support, we infer that

$$
M_{\psi} \text { is bounded from } H_{-\eta}^{2}\left(\mathbb{R}, \mathbb{C}^{2}\right) \text { to } H_{\eta}^{2}\left(\mathbb{R}, \mathbb{C}^{2}\right)
$$


Using (5.9) we obtain that

$$
\mathcal{T}_{ \pm}(\gamma)=\left(\mathcal{L}-\mathcal{L}^{\infty}\right)\left(\alpha_{ \pm}(\gamma) h_{ \pm}(\gamma)\right)+\left(\mathcal{L}^{\infty}-\gamma^{2}\right)\left(M_{\psi} \alpha_{ \pm}(\gamma) h_{ \pm}(\gamma)\right)
$$

From (5.10), (5.11) and (5.12) it follows that $\mathcal{T}_{ \pm}(\gamma) \in L_{\eta}^{2}\left(\mathbb{R}, \mathbb{C}^{2}\right)$ for all $\gamma \in B\left(0, \varepsilon_{0}\right)$, which proves that the maps $\mathcal{T}_{ \pm}$are well-defined. The analyticity of $\mathcal{T}_{ \pm}$follows from Lemma 5.1, (5.10), (5.11) and (5.12).

Remark 5.3. The operator $\mathcal{T}(0): H_{\eta}^{2}\left(\mathbb{R}, \mathbb{C}^{2}\right) \times \mathbb{C}^{2} \rightarrow L_{\eta}^{2}\left(\mathbb{R}, \mathbb{C}^{2}\right)$ is Fredholm with index 0 . The proof follows directly from Lemma 4.8 and a bordering lemma for Fredholm operators.

In the next lemma we prove the connection between the eigenvalue problem for $\mathcal{L}$ and $\mathcal{T}$.

Lemma 5.4. Under the assumptions of Theorem 1.1, the following assertion is true: if $\gamma \in$ $B\left(0, \varepsilon_{0}\right)$ and $\operatorname{Re}\left(\gamma^{2}\right)>0$ then

$$
\gamma^{2} \in \sigma_{\text {point }}(\mathcal{L}) \quad \text { if and only if } \quad 0 \in \sigma_{\text {point }}(\mathcal{T}(\gamma))
$$

Proof. First, we note that if $0 \in \sigma_{\text {point }}(\mathcal{T}(\gamma))$ then $\gamma^{2} \in \sigma_{\text {point }}(\mathcal{L})$ since the kernel of $\mathcal{T}(\gamma)$ readily yields eigenfunctions of $\mathcal{L}$ using (5.2).

Next, we show that the eigenvectors are necessarily of the form described in the ansatz (5.2). That is, we need to show that $\mathcal{L}-\gamma^{2}: H^{2}\left(\mathbb{R}, \mathbb{C}^{2}\right) \rightarrow L^{2}\left(\mathbb{R}, \mathbb{C}^{2}\right)$ is invertible provided that $0 \notin \sigma_{\text {point }}(\mathcal{T}(\gamma))$ and $\operatorname{Re}\left(\gamma^{2}\right)>0$.

If $\operatorname{Re}\left(\gamma^{2}\right)>0$ we have that $\gamma^{2} \notin \sigma_{\text {ess }}(\mathcal{L})$, which implies that $\mathcal{L}-\gamma^{2}: H^{2}\left(\mathbb{R}, \mathbb{C}^{2}\right) \rightarrow L^{2}\left(\mathbb{R}, \mathbb{C}^{2}\right)$ is Fredholm with index 0. Thus, to prove the lemma it is enough to show that the equation

$$
\left(\mathcal{L}-\gamma^{2}\right)\left(\begin{array}{l}
u \\
v
\end{array}\right)=\left(\begin{array}{l}
f_{1} \\
f_{2}
\end{array}\right),
$$

has solutions for every choice of $\left(f_{1}, f_{2}\right)^{\mathrm{T}}$ in a dense subspace of $L^{2}\left(\mathbb{R}, \mathbb{C}^{2}\right)$, for example $L_{\eta}^{2}\left(\mathbb{R}, \mathbb{C}^{2}\right)$. Since $\mathcal{T}(0)$ is Fredholm index 0 , by Remark 5.3, and since $\mathcal{T}(\cdot)$ is analytic on $B\left(0, \varepsilon_{0}\right)$, by Lemma 5.2 , we infer that we can choose $\varepsilon_{0}>0$ small enough such that $\mathcal{T}(\gamma)$ is Fredholm with index 0 for all $\gamma \in B\left(0, \varepsilon_{0}\right)$. Since, in addition, $0 \notin \sigma_{\text {point }}(\mathcal{T}(\gamma))$, it follows that equation (5.13) has a unique solution of the form (5.2) for any $\left(f_{1}, f_{2}\right)^{\mathrm{T}} \in L_{\eta}^{2}\left(\mathbb{R}, \mathbb{C}^{2}\right)$, proving the lemma.

The results proven so far in this section allow us to apply the Lyapunov-Schmidt reduction to $\mathcal{T}(\cdot)$ and obtain a bifurcation function that tracks the eigenvalues of $\mathcal{L}$ with positive real part. To conclude this section, we compute the kernels of $\mathcal{T}(0)$ and $\mathcal{T}(0)^{*}$.

Remark 5.5. Let $u \in C^{2}\left(\mathbb{R}, \mathbb{C}^{2}\right)$ be a smooth function such that $\mathcal{L} u \in L_{\eta}^{2}\left(\mathbb{R}, \mathbb{C}^{2}\right)$ and suppose that $u$ and $\left\langle D(\cdot) u^{\prime}, f\right\rangle$ have finite limits at $\pm \infty$ for all $f \in \operatorname{ker} \mathcal{L}^{*}$, where $\mathcal{L}^{*}$ is considered on $L_{-\eta}^{2}\left(\mathbb{R}, \mathbb{C}^{2}\right)$. Then, integrating twice by parts, and using Lemma 4.6 we obtain:

$$
\langle\mathcal{L} u, f\rangle_{L^{2}}=\left.\left\langle D(\cdot) u^{\prime}, f\right\rangle\right|_{-\infty} ^{\infty}-\left.\left\langle D(\cdot) u, f^{\prime}\right\rangle\right|_{-\infty} ^{\infty}
$$


Lemma 5.6. Under the assumptions of Theorem 1.1, the following assertions is true:

(i) If $\delta_{0} \neq 0$ then the kernel of $\mathcal{T}(0)$ on $H_{\eta}^{2}\left(\mathbb{R}, \mathbb{C}^{2}\right) \times \mathbb{C}^{2}$ is spanned by $\left(u_{x}^{*}, v_{x}^{*}, 0,0\right)^{\mathrm{T}}$, while the kernel of $\mathcal{T}(0)^{*}$ on $L_{-\eta}^{2}\left(\mathbb{R}, \mathbb{C}^{2}\right)$ is spanned $(1,0)^{\mathrm{T}}$;

(ii) If $\delta_{0}=0$ then the kernel of $\mathcal{T}(0)$ on $H_{\eta}^{2}\left(\mathbb{R}, \mathbb{C}^{2}\right) \times \mathbb{C}^{2}$ is spanned by $\left(u_{x}^{*}, v_{x}^{*}, 0,0\right)^{\mathrm{T}}$ and $\left(\partial_{\mu} \varphi\left(v^{*}, \mu_{0}\right)-\partial_{\mu} \varphi\left(v^{+}, \mu_{0}\right) \chi_{+}-\partial_{\mu} \varphi\left(v^{-}, \mu_{0}\right) \chi_{-}, 0,-\frac{\partial_{\mu} \varphi\left(v^{+}, \mu_{0}\right)}{g^{\prime}\left(v^{+}\right)},-\frac{\partial_{\mu} \varphi\left(v^{-}, \mu_{0}\right)}{g^{\prime}\left(v^{-}\right)}\right)^{\mathrm{T}}$, while the kernel of $\mathcal{T}(0)^{*}$ on $L_{-\eta}^{2}\left(\mathbb{R}, \mathbb{C}^{2}\right)$ is spanned $(1,0)^{\mathrm{T}}$ and $\left(0, v_{x}^{*}\right)^{\mathrm{T}}$.

Proof. We start the proof by computing $\mathcal{T}(0)^{*}$. Since

$$
\mathcal{T}(0)\left(\underline{w}, \beta_{1}, \beta_{2}\right)^{\mathrm{T}}=\mathcal{L}\left(\underline{w}+\beta_{1} \alpha_{+}(0) h_{+}(0)+\beta_{2} \alpha_{-}(0) h_{-}(0)\right)
$$

we obtain that

$$
\mathcal{T}(0)^{*}\left(f_{1}, f_{2}\right)^{\mathrm{T}}=\left(\mathcal{L}^{*}\left(f_{1}, f_{2}\right)^{\mathrm{T}},\left\langle\left(f_{1}, f_{2}\right)^{\mathrm{T}}, \mathcal{L}\left(\alpha_{+}(0) h_{+}(0)\right)\right\rangle_{L^{2}},\left\langle\left(f_{1}, f_{2}\right)^{\mathrm{T}}, \mathcal{L}\left(\alpha_{-}(0) h_{-}(0)\right)\right\rangle_{L^{2}}\right)^{\mathrm{T}}
$$

for all $\left(f_{1}, f_{2}\right)^{\mathrm{T}} \in L_{-\eta}^{2}\left(\mathbb{R}, \mathbb{C}^{2}\right)$. It follows that $\left(f_{1}, f_{2}\right)^{\mathrm{T}} \in \operatorname{ker} \mathcal{T}(0)^{*}$ on $L_{-\eta}^{2}\left(\mathbb{R}, \mathbb{C}^{2}\right)$ if and only if

$$
\left(f_{1}, f_{2}\right)^{\mathrm{T}} \in \operatorname{ker} \mathcal{L}^{*} \quad \text { on } \quad L_{-\eta}^{2}\left(\mathbb{R}, \mathbb{C}^{2}\right) \quad \text { and } \quad\left\langle\left(f_{1}, f_{2}\right)^{\mathrm{T}}, \mathcal{L}\left(\alpha_{ \pm}(0) h_{ \pm}(0)\right)\right\rangle_{L^{2}}=0 .
$$

From Lemma 4.6 we know that the elements of the kernel of $\mathcal{L}^{*}$ on $L_{-\eta}^{2}\left(\mathbb{R}, \mathbb{C}^{2}\right)$ are of the form

$$
\left(f_{1}, f_{2}\right)^{\mathrm{T}}=c_{1}(1,0)^{\mathrm{T}}+c_{2}\left(\bar{U}_{1}, 0\right)^{\mathrm{T}}+c_{3}\left(\bar{U}_{2}, v_{x}^{*}\right)^{\mathrm{T}},
$$

for some complex constants, $c_{j}, j=1,2,3$. Using Remark 5.5 we compute

$$
\begin{gathered}
\left\langle\mathcal{L}\left(\alpha_{ \pm}(0) h_{ \pm}(0)\right),(1,0)^{\mathrm{T}}\right\rangle_{L^{2}}=0, \quad\left\langle\mathcal{L}\left(\alpha_{ \pm}(0) h_{ \pm}(0)\right),\left(\bar{U}_{1}, 0\right)^{\mathrm{T}}\right\rangle_{L^{2}}= \pm \frac{a^{ \pm} g^{\prime}\left(v^{ \pm}\right)+\delta_{0} b^{ \pm}}{a^{ \pm} \partial_{\mu} \varphi\left(v^{ \pm}, \mu_{0}\right)} \\
\left\langle\mathcal{L}\left(\alpha_{ \pm}(0) h_{ \pm}(0)\right),\left(\bar{U}_{2}, v_{x}^{*}\right)^{\mathrm{T}}\right\rangle_{L^{2}}= \pm \frac{a^{ \pm} g^{\prime}\left(v^{ \pm}\right)+\delta_{0} b^{ \pm}}{a^{ \pm} \partial_{\mu} \varphi\left(v^{ \pm}, \mu_{0}\right)} \bar{M}^{ \pm}
\end{gathered}
$$

where $\bar{M}^{+}=\delta_{0} \int_{\mathbb{R}} \partial_{\mu} \varphi\left(v^{*}, \mu_{0}\right) v_{x}^{*}$ and $\bar{M}^{-}=0$.

From (5.15) it follows that $\left(f_{1}, f_{2}\right)^{\mathrm{T}}$ belongs to the kernel of $\mathcal{T}(0)$ if it satisfies (5.16) and

$$
\begin{gathered}
0=\left\langle\left(f_{1}, f_{2}\right)^{\mathrm{T}}, \mathcal{L}\left(\alpha_{+}(0) h_{+}(0)\right)\right\rangle_{L^{2}}=\frac{a^{+} g^{\prime}\left(v^{+}\right)+\delta_{0} b^{+}}{a^{+} \partial_{\mu} \varphi\left(v^{+}, \mu_{0}\right)}\left(c_{2}+c_{3} \delta_{0} \int_{\mathbb{R}} \partial_{\mu} \varphi\left(v^{*}, \mu_{0}\right) v_{x}^{*}\right) \\
0=\left\langle\left(f_{1}, f_{2}\right)^{\mathrm{T}}, \mathcal{L}\left(\alpha_{-}(0) h_{-}(0)\right)\right\rangle_{L^{2}}=-\frac{a^{-} g^{\prime}\left(v^{-}\right)+\delta_{0} b^{-}}{a^{-} \partial_{\mu} \varphi\left(v^{-}, \mu_{0}\right)} c_{2} .
\end{gathered}
$$

From the properties of $H$, Lemma 2.2 , it follows that $c_{2}=0$ and $\delta_{0} c_{3}=0$, which implies that

(i) If $\delta_{0} \neq 0$ then the kernel of $\mathcal{T}(0)^{*}$ is spanned by $(1,0)^{\mathrm{T}}$

(ii) If $\delta_{0}=0$ then the kernel of $\mathcal{T}(0)^{*}$ is spanned by $(1,0)^{\mathrm{T}}$ and $\left(\bar{U}_{2}, v_{x}^{*}\right)^{\mathrm{T}}=\left(0, v_{x}^{*}\right)^{\mathrm{T}}$. 
Moreover, since $\left(u_{x}^{*}, v_{x}^{*}\right)^{\mathrm{T}} \in \operatorname{ker} \mathcal{L}$ one can easily check that

$$
\left(u_{x}^{*}, v_{x}^{*}, 0,0\right)^{\mathrm{T}} \in \operatorname{ker} \mathcal{T}(0) .
$$

In addition, from Remark 5.3 we know that $\mathcal{T}(0)$ is Fredholm with index 0 , which implies that

$$
\operatorname{dim}(\operatorname{ker} \mathcal{T}(0))=\left\{\begin{array}{l}
1 \text { if } \delta_{0} \neq 0 \\
2 \text { if } \delta_{0}=0
\end{array}\right.
$$

If $\delta_{0}=0$, from Lemma 4.5 we know that $\left(\partial_{\mu} \varphi\left(v^{*}, \mu_{0}\right), 0\right)^{\mathrm{T}} \in$ ker $\mathcal{L}$ in $B U C\left(\mathbb{R}, \mathbb{C}^{2}\right)$ which implies that

$$
\left(\partial_{\mu} \varphi\left(v^{*}, \mu_{0}\right)-\partial_{\mu} \varphi\left(v^{+}, \mu_{0}\right) \chi_{+}-\partial_{\mu} \varphi\left(v^{-}, \mu_{0}\right) \chi_{-}, 0,-\frac{\partial_{\mu} \varphi\left(v^{+}, \mu_{0}\right)}{g^{\prime}\left(v^{+}\right)},-\frac{\partial_{\mu} \varphi\left(v^{-}, \mu_{0}\right)}{g^{\prime}\left(v^{-}\right)}\right)^{\mathrm{T}} \in \operatorname{ker} \mathcal{T}(0) .
$$

At this point, the lemma follows shortly from (5.17), (5.18) and (5.19).

Lemma 5.7. The results of this section remain true if we replace $\mathcal{L}$ with $\mathcal{L}(\delta)$, where $\delta$ is the homotopy parameter between 0 and $\delta_{0}$. Moreover, the function $\mathcal{T}$ can be extended to a smooth function on $\delta, \hat{\mathcal{T}}(\gamma, \delta): H_{\eta}^{2}\left(\mathbb{R}, \mathbb{C}^{2}\right) \times \mathbb{C}^{2} \rightarrow L_{\eta}^{2}\left(\mathbb{R}, \mathbb{C}^{2}\right)$ defined as follows:

$$
\hat{\mathcal{T}}(\gamma, \delta)\left(\underline{w}, \beta_{1}, \beta_{2}\right)^{\mathrm{T}}=\left(\mathcal{L}(\delta)-\gamma^{2}\right)\left[\underline{w}+\beta_{1} \hat{\alpha}_{+}(\gamma, \delta) \hat{h}_{+}(\gamma, \delta)+\beta_{2} \hat{\alpha}_{-}(\gamma, \delta) \hat{h}_{-}(\gamma, \delta)\right] .
$$

Here $\left[\hat{h}_{ \pm}(\gamma, \delta)\right](x)=\chi_{ \pm}(x) e^{\mp \hat{\nu}_{ \pm}(\gamma, \delta) x}$ and we recall the definition of $\hat{\alpha}_{ \pm}$and $\hat{\nu}_{ \pm}$in $(4.20)$.

Proof. By construction of the homotopy, properties of the layers are preserved from $\delta=1$ to $\delta=0$. This implies that $\mathcal{L}(\delta)$ possesses the same properties as $\mathcal{L}$. To establish smoothness (in fact analyticity) in $\delta$, first, note that $\mathcal{L}(\delta)$ has an affine dependence on $\delta$. Thus, we infer that the smooth dependence of $\hat{\mathcal{T}}$ on $\delta$ follows from the smooth dependence of $\hat{\alpha}_{ \pm}$and $\hat{\nu}_{ \pm}$on $\delta$. The latter was settled in Remark 4.11.

\section{Lyapunov-Schmidt reduction and eigenvalue problems}

In this section we move our attention to a general abstract result needed to solve the eigenvalue problems for $\mathcal{L}$ and $\mathcal{L}(\delta)$. We will show how to apply the Lyapunov-Schmidt reduction method to solve generalized eigenvalue problems. Let $X$ and $Y$ be two complex Hilbert spaces, $\Omega \subseteq \mathbb{R}^{m}$ a neighborhood of 0 and assume $S: B\left(0, \varepsilon_{0}\right) \times \Omega \rightarrow \mathcal{B}(X, Y)$ is an analytic function such that

$\left(S_{1}\right) \quad S(0,0)$ is Fredholm with index 0;

$\left(S_{2}\right) \operatorname{ker} S(0,0)=\left\{x_{j}: j=1, \ldots, d\right\} ;$

$\left(S_{3}\right) \operatorname{ker} S(0,0)^{*}=\left\{y_{j}: j=1, \ldots, d\right\}$. 
Here, by $B\left(0, \varepsilon_{0}\right)$ we denote the complex ball centered at 0 of radius $\varepsilon_{0}$ and by $\mathcal{B}(X, Y)$ the space of all bounded linear operators from $X$ to $Y$. The main purpose of this section is to discuss the generalized eigenvalue problem in the eigenvalue parameter $\gamma \in B\left(0, \varepsilon_{0}\right) \subset \mathbb{C}$,

$$
S(\gamma, \delta) x=0, \quad(\gamma, \delta) \in B\left(0, \varepsilon_{0}\right) \times \Omega
$$

and show how one can use the Lyapunov-Schmidt reduction method to construct an analytic bifurcation function that detects the eigenvalues of (6.1). Let $Z=\operatorname{ker} S(0,0)^{\perp}$ and let $Q_{0}$ be the orthogonal projection onto $\operatorname{im} S(0,0)$. It follows that equation (6.1) is equivalent to the generalized eigenvalue system

$$
\left\{\begin{array}{l}
Q_{0} S(\gamma, \delta) x=0 \\
\left\langle S(\gamma, \delta) x, y_{j}\right\rangle=0, j=1, \ldots, d .
\end{array}\right.
$$

In the system above we make the substitution $x=z+\sum_{j=1}^{d} a_{j} x_{j}, z \in Z, a_{1}, \ldots, a_{d} \in \mathbb{C}$ and solve the first equation for $z$ using the Implicit Function Theorem. Therefore, we introduce first the function $F: Z \times \mathbb{C}^{d} \times B\left(0, \varepsilon_{0}\right) \times \Omega \rightarrow \operatorname{im} S(0,0)$ by

$$
F\left(z, a_{1}, \ldots, a_{d}, \gamma, \delta\right)=Q_{0} S(\gamma, \delta)\left(z+\sum_{j=1}^{d} a_{j} x_{j}\right)
$$

Also, denote by $\mathrm{e}_{j}, j=1, \ldots, d$, the vectors of the canonical basis of $\mathbb{R}^{d}$.

Lemma 6.1. Assume $S: B\left(0, \varepsilon_{0}\right) \times \Omega \rightarrow \mathcal{B}(X, Y)$ is an analytic function satisfying conditions $\left(S_{1}\right)-\left(S_{3}\right)$.

(i) After eventually shrinking $B\left(0, \varepsilon_{0}\right) \times \Omega$, there are analytic functions $z_{1}, \ldots, z_{d}: B\left(0, \varepsilon_{0}\right) \times$ $\Omega \rightarrow Z$ such that

$\left(z_{1}\right) z_{j}(0,0)=0, j=1, \ldots, d ;$

$\left(z_{2}\right)$ locally, the equation $F\left(z, \mathrm{e}_{j}, \gamma, \delta\right)=0$ has the unique solution $z=z_{j}(\gamma, \delta)$ for any $j=1, \ldots, d$;

(ii) The partial derivatives of the functions $z_{j}, j=1, \ldots, d$ can be computed by the formulas

$$
\begin{aligned}
& \frac{\partial z_{j}}{\partial \gamma}(0,0)=-\left(Q_{0} S(0,0)_{\mid Z}\right)^{-1} Q_{0} \frac{\partial S}{\partial \gamma}(0,0) x_{j} \\
& \frac{\partial z_{j}}{\partial \delta_{k}}(0,0)=-\left(Q_{0} S(0,0)_{\mid Z}\right)^{-1} Q_{0} \frac{\partial S}{\partial \delta_{k}}(0,0) x_{j}
\end{aligned}
$$

for all $j=1, \ldots, d, k=1, \ldots, m$. If $\frac{\partial z_{j}}{\partial \gamma}(0,0)=0$ then

$$
\frac{\partial^{2} z_{j}}{\partial^{2} \gamma}(0,0)=-\left(Q_{0} S(0,0)_{\mid Z}\right)^{-1} Q_{0} \frac{\partial^{2} S}{\partial^{2} \gamma}(0,0) x_{j}
$$


Proof. To prove (i) all we need to check is that we can apply the Implicit Functions Theorem. From $\left(S_{2}\right)$ it follows that

$$
F\left(0, \mathrm{e}_{j}, 0,0\right)=Q_{0} S(0,0) x_{j}=0 \text { for all } j=1, \ldots, d .
$$

From the definition of the subspace $Z$ and the projection $Q_{0}$ it follows that $\frac{\partial F}{\partial z}\left(0, \mathrm{e}_{j}, 0,0\right)=$ $Q_{0} S(0,0)_{\mid Z}$ is an invertible operator with bounded inverse from $Z$ to $\mathrm{im} S(0,0)$. To check (ii) one only needs to differentiate in the equation $F\left(z_{j}(\gamma, \delta), \mathrm{e}_{j}, \gamma, \delta\right)=0$.

Now we are ready to state the main result of this section:

Lemma 6.2. Assume $S: B\left(0, \varepsilon_{0}\right) \times \Omega \rightarrow \mathcal{B}(X, Y)$ is an analytic function satisfying conditions $\left(S_{1}\right)-\left(S_{3}\right)$. If $(\gamma, \delta) \in B\left(0, \varepsilon_{0}\right) \times \Omega$ then $0 \in \sigma_{\text {point }}(S(\gamma, \delta))$ if and only if

$$
E(\gamma, \delta)=\operatorname{det}\left[\left\langle S(\gamma, \delta)\left(x_{j}+z_{j}(\gamma, \delta)\right), y_{\ell}\right\rangle\right]_{1 \leq j, \ell \leq d}=0 .
$$

Proof. From Lemma 6.1 it follows that for each $(\gamma, \delta) \in B\left(0, \varepsilon_{0}\right) \times \Omega$ the unique solution of the first equation of $(6.2)$ is $z=\sum_{j=1}^{d} a_{j} z_{j}(\gamma, \delta)$. It follows that the eigenvalue problem (6.1) is equivalent to the system

$$
\sum_{j=1}^{d} a_{j}\left\langle S(\gamma, \delta)\left(x_{j}+z_{j}(\gamma, \delta)\right), y_{\ell}\right\rangle=0, \ell=1, \ldots, d,
$$

which has a non-zero solution if and only if $E(\gamma, \delta)=0$, proving the lemma.

\section{Expansions of the bifurcation function}

In this section we compute the expansion of a bifurcation function $E$ that determines the solvability of the eigenvalue problem (5.1). We assume that (1.5) has an exponentially localized layer solution $\left(u^{*}, v^{*}\right)$ at $\delta=\delta_{0}$ and that the essential spectrum of the operator $\mathcal{L}$ is stable.

In what follows we consider the eigenvalue problem

$$
\mathcal{T}(\gamma)\left(\underline{w}, \beta_{1}, \beta_{2}\right)^{\mathrm{T}}=0 .
$$

on the Hilbert space $H_{\eta}^{2}\left(\mathbb{R}, \mathbb{C}^{2}\right) \times \mathbb{C}^{2}$. From Lemma 6.2 we know that there is an analytic function $E: B\left(0, \varepsilon_{0}\right) \rightarrow \mathbb{C}$ such that $E(\gamma)=0$ if and only if $0 \in \sigma_{\text {point }}(\mathcal{T}(\gamma))$. In the next lemmas we compute the leading order terms in the expansion of $E$.

Lemma 7.1. Under the assumptions of Theorem 1.1, and if $\delta_{0} \neq 0$, the bifurcation function E has the expansion

$$
E(\gamma)=c_{2} \gamma^{2}+c_{3} \gamma^{3}+\mathcal{O}\left(\gamma^{4}\right)
$$

where $c_{2}=-\left(u^{+}-u^{-}\right)$and

$$
\begin{gathered}
c_{3}=\frac{\nu_{+}^{\prime}(0) a^{+} \partial_{\mu} \varphi\left(v^{+}, \mu_{0}\right)+\nu_{-}^{\prime}(0) a^{-} \partial_{\mu} \varphi\left(v^{-}, \mu_{0}\right)}{\delta_{0} \int_{\mathbb{R}} \partial_{\mu} \varphi\left(v^{*}, \mu_{0}\right) v_{x}^{*}}\left(\left\|v_{x}^{*}\right\|_{2}^{2}+\left\langle u_{x}^{*}-\frac{1}{\eta}\left(u^{+}-u^{-}\right) e^{-2 \eta|\cdot|}, \bar{U}_{2}\right\rangle_{L^{2}}\right) \\
+\nu_{-}^{\prime}(0) a^{-} \partial_{\mu} \varphi\left(v^{-}, \mu_{0}\right)\left\langle u_{x}^{*}-\frac{1}{\eta}\left(u^{+}-u^{-}\right) e^{-2 \eta|\cdot|}, \bar{U}_{1}\right\rangle_{L^{2}} .
\end{gathered}
$$


Proof. Throughout the proof we are using the notations from Lemma 6.2. From Lemma 5.6 we have that $\operatorname{ker} \mathcal{T}(0)$ is spanned by $\left(u_{x}^{*}, v_{x}^{*}, 0,0\right)^{\mathrm{T}}$ and $\operatorname{ker} \mathcal{T}(0)^{*}$ is spanned by $(1,0)^{\mathrm{T}}$. It follows that

$$
\operatorname{im} \mathcal{T}(0)=\left\{\left(f_{1}, f_{2}\right)^{\mathrm{T}} \in L_{\eta}^{2}\left(\mathbb{R}, \mathbb{C}^{2}\right): \int_{\mathbb{R}} f_{1}(x) \mathrm{d} x=0\right\} .
$$

Since

$$
\int_{\mathbb{R}} f_{1}(x) \mathrm{d} x=\left\langle f_{1}, e^{-2 \eta|\cdot|}\right\rangle_{L_{\eta}^{2}} \quad \text { for all } \quad f_{1} \in L_{\eta}^{2}\left(\mathbb{R}, \mathbb{C}^{2}\right),
$$

we have that $Q_{0}$, the projection onto $\operatorname{im} \mathcal{T}(0)$ along $\operatorname{ker} \mathcal{T}(0)^{*}$, is given by

$$
\begin{aligned}
Q_{0}\left(f_{1}, f_{2}\right)^{\mathrm{T}} & =\left(f_{1}, f_{2}\right)^{\mathrm{T}}-\frac{\left\langle\left(f_{1}, f_{2}\right)^{\mathrm{T}},\left(e^{-2 \eta|\cdot|}, 0\right)^{\mathrm{T}}\right\rangle_{L_{\eta}^{2}}}{\left\langle\left(e^{-2 \eta|\cdot|}, 0\right)^{\mathrm{T}},\left(e^{-2 \eta|\cdot|}, 0\right)^{\mathrm{T}}\right\rangle_{L_{\eta}^{2}}}\left(e^{-2 \eta|\cdot|}, 0\right)^{\mathrm{T}} \\
& =\left(f_{1}, f_{2}\right)^{\mathrm{T}}-\frac{1}{\eta}\left\langle f_{1}, e^{-2 \eta|\cdot|}\right\rangle_{L_{\eta}^{2}}\left(e^{-2 \eta|\cdot|}, 0\right)^{\mathrm{T}} .
\end{aligned}
$$

From Lemma 6.2 we know that

$$
\begin{aligned}
E(\gamma) & =\left\langle\mathcal{T}(\gamma)\left[\left(u_{x}^{*}, v_{x}^{*}, 0,0\right)^{\mathrm{T}}+z_{1}(\gamma)\right],(1,0)^{\mathrm{T}}\right\rangle_{L^{2}} \\
& =\left\langle\left(\mathcal{L}-\gamma^{2}\right)\left(u_{x}^{*}, v_{x}^{*}\right)^{\mathrm{T}},(1,0)^{\mathrm{T}}\right\rangle_{L^{2}}+\left\langle\mathcal{T}(\gamma) z_{1}(\gamma),(1,0)^{\mathrm{T}}\right\rangle_{L^{2}} \\
& =-\gamma^{2}\left\langle\left(u_{x}^{*}, v_{x}^{*}\right)^{\mathrm{T}},(1,0)^{\mathrm{T}}\right\rangle_{L^{2}}+\left\langle\mathcal{T}(\gamma) z_{1}(\gamma),(1,0)^{\mathrm{T}}\right\rangle_{L^{2}} \\
& =-\left(u^{+}-u^{-}\right) \gamma^{2}+\left\langle\mathcal{T}(\gamma) z_{1}(\gamma),(1,0)^{\mathrm{T}}\right\rangle_{L^{2}} .
\end{aligned}
$$

Next, we find the expansion for $z_{1}(\gamma), \gamma \in B\left(0, \varepsilon_{0}\right)$. From Lemma $6.1(\mathrm{i})$ we have that $z_{1}(0)=0$. Moreover,

$$
\mathcal{T}^{\prime}(0)\left(\underline{w}, \beta_{1}, \beta_{2}\right)=\mathcal{L}\left[\beta_{1}\left(\alpha_{+} h_{+}\right)^{\prime}(0)+\beta_{2}\left(\alpha_{-} h_{-}\right)^{\prime}(0)\right],
$$

which implies that $\mathcal{T}^{\prime}(0)\left(u_{x}^{*}, v_{x}^{*}, 0,0\right)^{\mathrm{T}}=0$. From Lemma $6.1(\mathrm{ii})$ it follows that $z_{1}^{\prime}(0)=0$. Thus,

$$
z_{1}(\gamma)=\frac{1}{2} z_{1}^{\prime \prime}(0) \gamma^{2}+\mathcal{O}\left(\gamma^{3}\right)
$$

which implies that

$$
\begin{aligned}
\left\langle\mathcal{T}(\gamma) z_{1}(\gamma),(1,0)^{\mathrm{T}}\right\rangle_{L^{2}} & =\left\langle\left(\mathcal{T}(0)+\gamma \mathcal{T}^{\prime}(0)\right) z_{1}(\gamma),(1,0)^{\mathrm{T}}\right\rangle_{L^{2}}+\mathcal{O}\left(\gamma^{4}\right) \\
& =\left\langle\mathcal{T}(0) z_{1}(\gamma),(1,0)^{\mathrm{T}}\right\rangle_{L^{2}}+\frac{1}{2} \gamma^{3}\left\langle\mathcal{T}^{\prime}(0) z_{1}^{\prime \prime}(0),(1,0)^{\mathrm{T}}\right\rangle_{L^{2}}+\mathcal{O}\left(\gamma^{4}\right) \\
& =\left\langle z_{1}(\gamma), \mathcal{T}(0)^{*}(1,0)^{\mathrm{T}}\right\rangle_{L^{2}}+\frac{1}{2} \gamma^{3}\left\langle z_{1}^{\prime \prime}(0), \mathcal{T}^{\prime}(0)^{*}(1,0)^{\mathrm{T}}\right\rangle_{L^{2}}+\mathcal{O}\left(\gamma^{4}\right) \\
& =\frac{1}{2} \gamma^{3}\left\langle z_{1}^{\prime \prime}(0), \mathcal{T}^{\prime}(0)^{*}(1,0)^{\mathrm{T}}\right\rangle_{L^{2}}+\mathcal{O}\left(\gamma^{4}\right) .
\end{aligned}
$$

From (7.3) and (7.6) it follows that $c_{2}=-\left(u^{+}-u^{-}\right)$. Next, we compute $\mathcal{T}^{\prime}(0)$ and the components of $z_{1}^{\prime \prime}(0)$. From (7.4) it follows that

$$
\mathcal{T}^{\prime}(0)^{*}\left(f_{1}, f_{2}\right)^{\mathrm{T}}=\left(0,\left\langle\left(f_{1}, f_{2}\right)^{\mathrm{T}}, \mathcal{L}\left(\alpha_{+} h_{+}\right)^{\prime}(0)\right\rangle_{L^{2}},\left\langle\left(f_{1}, f_{2}\right)^{\mathrm{T}}, \mathcal{L}\left(\alpha_{-} h_{-}\right)^{\prime}(0)\right\rangle_{L^{2}}\right)^{\mathrm{T}} .
$$

Moreover, from Remark 5.5 we have that

$$
\left\langle\mathcal{L}\left(\alpha_{ \pm} h_{ \pm}\right)^{\prime}(0),(1,0)^{\mathrm{T}}\right\rangle_{L^{2}}=-\left.\left(\delta_{0} b^{*}+a^{*} g^{\prime}\left(v^{ \pm}\right)\right)\left(\chi_{ \pm} \mathrm{id}_{\mathbb{R}}\right)^{\prime}\right|_{-\infty} ^{\infty} \cdot\left( \pm \nu_{ \pm}^{\prime}(0)\right)
$$




$$
=\nu_{ \pm}^{\prime}(0)\left(a^{ \pm} g^{\prime}\left(v^{ \pm}\right)+\delta_{0} b^{ \pm}\right)
$$

Using Lemma 6.1(ii) again, we have that

$$
\mathcal{T}(0) z_{1}^{\prime \prime}(0)=-Q_{0} \mathcal{T}^{\prime \prime}(0)\left(u_{x}^{*}, v_{x}^{*}, 0,0\right)^{\mathrm{T}} .
$$

We know that $z_{1}^{\prime \prime}(0)=\left(\underline{w}_{0}^{*}, m_{1}, m_{2}\right)^{\mathrm{T}}$, where $\underline{w}_{0}^{*} \in H_{\eta}^{2}\left(\mathbb{R}, \mathbb{C}^{2}\right)$ and $m_{1}, m_{2} \in \mathbb{C}$. Using (7.6), (7.7) and (7.8), we note that to finish the proof of the lemma it is enough to compute $m_{1}$ and $m_{2}$. Since $\mathcal{T}^{\prime \prime}(0)(\underline{w}, 0,0)^{\mathrm{T}}=-2 \underline{w}$ for all $\underline{w} \in H_{\eta}^{2}\left(\mathbb{R}, \mathbb{C}^{2}\right)$ we obtain that

$$
Q_{0} \mathcal{T}^{\prime \prime}(0)\left(u_{x}^{*}, v_{x}^{*}, 0,0\right)^{\mathrm{T}}=-2 Q_{0}\left(u_{x}^{*}, v_{x}^{*}\right)^{\mathrm{T}}=-2\left[\left(u_{x}^{*}, v_{x}^{*}\right)^{\mathrm{T}}-\frac{1}{\eta}\left(u^{+}-u^{-}\right)\left(e^{-2 \eta|\cdot|}, 0\right)^{\mathrm{T}}\right] .
$$

Plugging the above expression into (7.9), we obtain that

$$
\mathcal{L} \underline{w}_{0}^{*}+m_{1} \mathcal{L}\left(\alpha_{+}(0) h_{+}(0)\right)+m_{2} \mathcal{L}\left(\alpha_{-}(0) h_{-}(0)\right)=2\left[\left(u_{x}^{*}, v_{x}^{*}\right)^{\mathrm{T}}-\frac{1}{\eta}\left(u^{+}-u^{-}\right)\left(e^{-2 \eta|\cdot|}, 0\right)^{\mathrm{T}}\right] .
$$

Since $\underline{w}_{0}^{*} \in H_{\eta}^{2}\left(\mathbb{R}, \mathbb{C}^{2}\right)$ and $\left(\bar{U}_{1}, 0\right)^{\mathrm{T}},\left(\bar{U}_{2}, v_{x}^{*}\right)^{\mathrm{T}} \in \operatorname{ker} \mathcal{L}^{*}$ on $L_{-\eta}^{2}\left(\mathbb{R}, \mathbb{C}^{2}\right)$ we infer that

$$
\left\langle\mathcal{L} \underline{w}_{0}^{*},\left(\bar{U}_{1}, 0\right)^{\mathrm{T}}\right\rangle_{L^{2}}=0 \text { and }\left\langle\mathcal{L} \underline{w}_{0}^{*},\left(\bar{U}_{2}, v_{x}^{*}\right)^{\mathrm{T}}\right\rangle_{L^{2}}=0 .
$$

Taking scalar products with $\left(\bar{U}_{1}, 0\right)^{\mathrm{T}}$ and $\left(\bar{U}_{2}, v_{x}^{*}\right)^{\mathrm{T}}$, respectively, in (7.10) we obtain that

$$
\begin{gathered}
m_{1}\left\langle\mathcal{L}\left(\alpha_{+}(0) h_{+}(0)\right),\left(\bar{U}_{1}, 0\right)^{\mathrm{T}}\right\rangle_{L^{2}}+m_{2}\left\langle\mathcal{L}\left(\alpha_{-}(0) h_{-}(0)\right),\left(\bar{U}_{1}, 0\right)^{\mathrm{T}}\right\rangle_{L^{2}}= \\
=2\left\langle u_{x}^{*}-\frac{1}{\eta}\left(u^{+}-u^{-}\right) e^{-2 \eta|\cdot|}, \bar{U}_{1}\right\rangle_{L^{2}} ; \\
m_{1}\left\langle\mathcal{L}\left(\alpha_{+}(0) h_{+}(0)\right),\left(\bar{U}_{2}, v_{x}^{*}\right)^{\mathrm{T}}\right\rangle_{L^{2}}+m_{2}\left\langle\mathcal{L}\left(\alpha_{-}(0) h_{-}(0)\right),\left(\bar{U}_{2}, v_{x}^{*}\right)^{\mathrm{T}}\right\rangle_{L^{2}}= \\
=2\left\langle u_{x}^{*}-\frac{1}{\eta}\left(u^{+}-u^{-}\right) e^{-2 \eta|\cdot|}, \bar{U}_{2}\right\rangle_{L^{2}}+2\left\|v_{x}^{*}\right\|_{2}^{2} .
\end{gathered}
$$

Using Remark 5.5 we can now compute the coefficients of $m_{j}, j=1,2$, in the equations above as follows:

$$
\begin{gathered}
\left\langle\mathcal{L}\left(\alpha_{ \pm}(0) h_{ \pm}(0)\right),\left(\bar{U}_{1}, 0\right)^{\mathrm{T}}\right\rangle_{L^{2}}= \pm \frac{a^{ \pm} g^{\prime}\left(v^{ \pm}\right)+\delta_{0} b^{ \pm}}{a^{ \pm} \partial_{\mu} \varphi\left(v^{ \pm}, \mu_{0}\right)} \\
\left\langle\mathcal{L}\left(\alpha_{ \pm}(0) h_{ \pm}(0)\right),\left(\bar{U}_{2}, v_{x}^{*}\right)^{\mathrm{T}}\right\rangle_{L^{2}}=\mp \frac{a^{ \pm} g^{\prime}\left(v^{ \pm}\right)+\delta_{0} b^{ \pm}}{a^{ \pm} \partial_{\mu} \varphi\left(v^{ \pm}, \mu_{0}\right)} \bar{M}^{ \pm},
\end{gathered}
$$

where $\bar{M}^{+}=\delta_{0} \int_{\mathbb{R}} \partial_{\mu} \varphi\left(v^{*}, \mu_{0}\right) v_{x}^{*}$ and $\bar{M}^{-}=0$.

Solving the above system for $m_{1}$ and $m_{2}$, we obtain the expression of the coefficient of $\gamma^{3}$ in the expansion of $E$, proving the lemma.

Remark 7.2. Lemma 7.1 is also true if we replace $\mathcal{L}$ with $\mathcal{L}(\delta)$, and $\delta_{0}$ with $\delta$, where here $\delta$ belongs to a compact subinterval of the homotopy interval that stays away from 0. 
To track the behavior of eigenvalues in a neighborhood of 0 , we use Lemma 5.7, in particular the result from Lemma 5.4 for $\mathcal{L}(\delta)$ instead of $\mathcal{L}$ and solve the eigenvalue problem

$$
\hat{\mathcal{T}}(\gamma, \delta)\left(\underline{w}, \beta_{1}, \beta_{2}\right)^{\mathrm{T}}=0
$$

on the Hilbert space $H_{\eta}^{2}\left(\mathbb{R}, \mathbb{C}^{2}\right) \times \mathbb{C}^{2}$, where $\hat{\mathcal{T}}$ was defined in (5.20). To compute the expansion of the bifurcation function $\hat{E}$, corresponding to the eigenvalue problem (7.13), constructed by using Lemma 6.2, we start by computing the expansion of $\hat{E}(\gamma, 0)$, which is equal to $E(\gamma)$ in the case when $\delta_{0}=0$.

Lemma 7.3. Under the assumptions of Theorem 1.1, and if $\delta_{0}=0$, the bifurcation function $E$ (and $\hat{E}(\cdot, 0))$ has the expansion

$$
E(\gamma)=-\left(\left(a^{+}\right)^{1 / 2} \partial_{\mu} \varphi\left(v^{+}, \mu_{0}\right)+\left(a^{-}\right)^{1 / 2} \partial_{\mu} \varphi\left(v^{-}, \mu_{0}\right)\right) \gamma^{3}+\mathcal{O}\left(\gamma^{4}\right)
$$

Proof. Similar to the previous lemma, throughout this proof we will be using the notation from Section 6. From Lemma 5.6 we have that

$$
\operatorname{ker} \mathcal{T}(0)=\operatorname{Sp}\left\{x_{1}, x_{2}\right\} \quad \text { and } \operatorname{ker} \mathcal{T}(0)^{*}=\operatorname{Sp}\left\{y_{1}, y_{2}\right\}
$$

where $y_{1}=(1,0)^{\mathrm{T}}, y_{2}=\left(0, v_{x}^{*}\right)^{\mathrm{T}}, x_{1}=\left(u_{x}^{*}, v_{x}^{*}, 0,0\right)^{\mathrm{T}}$ and

$$
x_{2}=\left(\partial_{\mu} \varphi\left(v^{*}, \mu_{0}\right)-\partial_{\mu} \varphi\left(v^{+}, \mu_{0}\right) \chi_{+}-\partial_{\mu} \varphi\left(v^{-}, \mu_{0}\right) \chi_{-}, 0,-\frac{\partial_{\mu} \varphi\left(v^{+}, \mu_{0}\right)}{g^{\prime}\left(v^{+}\right)},-\frac{\partial_{\mu} \varphi\left(v^{-}, \mu_{0}\right)}{g^{\prime}\left(v^{-}\right)}\right)^{\mathrm{T}} .
$$

From Lemma 6.2 we know that $E(\gamma)=\operatorname{det}\left(a_{j k}(\gamma)\right)_{1 \leq j, k \leq 2}$, where

$$
a_{j k}(\gamma)=\left\langle\mathcal{T}(\gamma)\left(x_{j}+z_{j}(\gamma)\right), y_{k}\right\rangle_{L^{2}} \quad \text { for all } \quad j, k=1,2
$$

We start by computing

$$
\begin{aligned}
\left\langle\mathcal{T}(\gamma) x_{1}, y_{1}\right\rangle_{L^{2}} & =\left\langle\left(\mathcal{L}-\gamma^{2}\right)\left(u_{x}^{*}, v_{x}^{*}\right)^{\mathrm{T}},(1,0)^{\mathrm{T}}\right\rangle_{L^{2}}=-\gamma^{2}\left\langle\left(u_{x}^{*}, v_{x}^{*}\right)^{\mathrm{T}},(1,0)^{\mathrm{T}}\right\rangle_{L^{2}} \\
& =-\left(u^{+}-u^{-}\right) \gamma^{2} . \\
\left\langle\mathcal{T}(\gamma) x_{1}, y_{2}\right\rangle_{L^{2}} & =\left\langle\left(\mathcal{L}-\gamma^{2}\right)\left(u_{x}^{*}, v_{x}^{*}\right)^{\mathrm{T}},\left(0, v_{x}^{*}\right)^{\mathrm{T}}\right\rangle_{L^{2}}=-\gamma^{2}\left\langle\left(u_{x}^{*}, v_{x}^{*}\right)^{\mathrm{T}},\left(0, v_{x}^{*}\right)^{\mathrm{T}}\right\rangle_{L^{2}} \\
& =-\left\|v_{x}^{*}\right\|_{2}^{2} \gamma^{2} .
\end{aligned}
$$

Denoting by $W_{ \pm}=-\frac{\partial_{\mu} \varphi\left(v^{ \pm}, \mu_{0}\right)}{g^{\prime}\left(v^{ \pm}\right)}$and using Remark 5.5, we have that

$$
\left\langle\mathcal{L}\left(\alpha_{ \pm} h_{ \pm}\right)^{\prime}(0),(1,0)^{\mathrm{T}}\right\rangle_{L^{2}}=\left(a^{ \pm}\right)^{1 / 2} g^{\prime}\left(v^{ \pm}\right)
$$

which implies that

$$
\begin{aligned}
\left\langle\mathcal{T}(\gamma) x_{2}, y_{1}\right\rangle_{L^{2}} & =\gamma\left\langle\mathcal{T}^{\prime}(0) x_{2},(1,0)^{\mathrm{T}}\right\rangle_{L^{2}}+\mathcal{O}\left(\gamma^{2}\right) \\
& =\left\langle\mathcal{L}\left[W_{+}\left(\alpha_{+} h_{+}\right)^{\prime}(0)+W_{-}\left(\alpha_{-} h_{-}\right)^{\prime}(0)\right],(1,0)^{\mathrm{T}}\right\rangle_{L^{2}} \gamma+\mathcal{O}\left(\gamma^{2}\right) \\
& =\left[W_{+}\left(a^{+}\right)^{1 / 2} g^{\prime}\left(v^{+}\right)+W_{-}\left(a^{-}\right)^{1 / 2} g^{\prime}\left(v^{-}\right)\right] \gamma+\mathcal{O}\left(\gamma^{2}\right)
\end{aligned}
$$




$$
=-\left[\left(a^{+}\right)^{1 / 2} \partial_{\mu} \varphi\left(v^{+}, \mu_{0}\right)+\left(a^{-}\right)^{1 / 2} \partial_{\mu} \varphi\left(v^{-}, \mu_{0}\right)\right] \gamma+\mathcal{O}\left(\gamma^{2}\right)
$$

Similarly, from Remark 5.5 we have that

$$
\left\langle\mathcal{L}\left(\alpha_{ \pm} h_{ \pm}\right)^{\prime}(0),\left(0, v_{x}^{*}\right)^{\mathrm{T}}\right\rangle_{L^{2}}=0
$$

and thus,

$$
\begin{aligned}
\left\langle\mathcal{T}(\gamma) x_{2}, y_{2}\right\rangle_{L^{2}} & =\gamma\left\langle\mathcal{T}^{\prime}(0) x_{2},\left(0, v_{x}^{*}\right)^{\mathrm{T}}\right\rangle_{L^{2}}+\mathcal{O}\left(\gamma^{2}\right) \\
& =\left\langle\mathcal{L}\left[W_{+}\left(\alpha_{+} h_{+}\right)^{\prime}(0)+W_{-}\left(\alpha_{-} h_{-}\right)^{\prime}(0)\right],\left(0, v_{x}^{*}\right)^{\mathrm{T}}\right\rangle_{L^{2}} \gamma+\mathcal{O}\left(\gamma^{2}\right) \\
& =\mathcal{O}\left(\gamma^{2}\right) .
\end{aligned}
$$

From (7.4) it follows that

$$
\mathcal{T}^{\prime}(0) x_{1}=\mathcal{T}^{\prime}(0)\left(u_{x}^{*}, v_{x}^{*}, 0,0\right)^{\mathrm{T}}=0,
$$

which implies that $z_{1}^{\prime}(0)=0$. Since, from Lemma 6.1 we have in addition that $z_{1}(0)=0$, we infer that $z_{1}(\gamma)=\mathcal{O}\left(\gamma^{2}\right)$. Next, we estimate

$$
\begin{aligned}
\left\langle\mathcal{T}(\gamma) z_{1}(\gamma), y_{k}\right\rangle_{L^{2}} & =\left\langle\mathcal{T}(0) z_{1}(\gamma), y_{k}\right\rangle_{L^{2}}+\left\langle(\mathcal{T}(\gamma)-\mathcal{T}(0)) z_{1}(\gamma), y_{k}\right\rangle_{L^{2}} \\
& =\left\langle z_{1}(\gamma), \mathcal{T}(0)^{*} y_{k}\right\rangle_{L^{2}}+\left\langle(\mathcal{T}(\gamma)-\mathcal{T}(0)) z_{1}(\gamma), y_{k}\right\rangle_{L^{2}} \\
& =\left\langle(\mathcal{T}(\gamma)-\mathcal{T}(0)) z_{1}(\gamma), y_{k}\right\rangle_{L^{2}}=\mathcal{O}\left(\gamma^{3}\right), k=1,2
\end{aligned}
$$

Similarly, from Lemma $6.1(\mathrm{i})$ we have that $z_{2}(0)=0$, which implies that $z_{2}(\gamma)=\mathcal{O}(\gamma)$. We estimate

$$
\begin{aligned}
\left\langle\mathcal{T}(\gamma) z_{2}(\gamma), y_{k}\right\rangle_{L^{2}} & =\left\langle\mathcal{T}(0) z_{2}(\gamma), y_{k}\right\rangle_{L^{2}}+\left\langle(\mathcal{T}(\gamma)-\mathcal{T}(0)) z_{2}(\gamma), y_{k}\right\rangle_{L^{2}} \\
& =\left\langle z_{2}(\gamma), \mathcal{T}(0)^{*} y_{k}\right\rangle_{L^{2}}+\left\langle(\mathcal{T}(\gamma)-\mathcal{T}(0)) z_{2}(\gamma), y_{k}\right\rangle_{L^{2}} \\
& =\left\langle(\mathcal{T}(\gamma)-\mathcal{T}(0)) z_{2}(\gamma), y_{k}\right\rangle_{L^{2}}=\mathcal{O}\left(\gamma^{2}\right), k=1,2
\end{aligned}
$$

At this point, the lemma follows shortly from (7.15)-(7.21).

In the next lemma we finish the computation of the expansion of $\hat{E}$.

Lemma 7.4. Under the assumptions of Theorem 1.1, the bifurcation function $\hat{E}$ has the expansion

$$
\hat{E}(\gamma, \delta)=c_{2} \delta \gamma^{2}+c_{3} \gamma^{3}+\mathcal{O}(4)
$$

where

$$
c_{2}=-\left(u^{+}-u^{-}\right) \int_{v^{-}}^{v^{+}} \partial_{\mu} \varphi\left(v, \mu_{0}\right) \mathrm{d} v, c_{3}=-\left[\left(a^{+}\right)^{1 / 2} \partial_{\mu} \varphi\left(v^{+}, \mu_{0}\right)+\left(a^{-}\right)^{1 / 2} \partial_{\mu} \varphi\left(v^{-}, \mu_{0}\right)\right]\left\|v_{x}^{*}\right\|_{2}^{2} .
$$

Proof. Like in the previous lemmas, throughout this proof we will use the notation from Section 6. Using Lemma 5.6 again we infer that

$$
\operatorname{ker} \hat{\mathcal{T}}(0,0)=\operatorname{ker} \mathcal{T}(0)=\operatorname{Sp}\left\{x_{1}, x_{2}\right\} \quad \text { and } \quad \operatorname{ker} \hat{\mathcal{T}}(0,0)^{*}=\operatorname{ker} \mathcal{T}(0)^{*}=\operatorname{Sp}\left\{y_{1}, y_{2}\right\} .
$$


Here $y_{1}=(1,0)^{\mathrm{T}}, y_{2}=\left(0, v_{x}^{*}\right)^{\mathrm{T}}, x_{1}=\left(u_{x}^{*}, v_{x}^{*}, 0,0\right)^{\mathrm{T}}$ and $x_{2}=\left(U-U_{+} \chi_{+}-U_{-} \chi_{-}, 0, W_{+}, W_{-}\right)^{\mathrm{T}}$, where

$$
U=\partial_{\mu} \varphi\left(v^{*}, \mu_{0}\right), \quad U_{ \pm}=\partial_{\mu} \varphi\left(v^{ \pm}, \mu_{0}\right), \quad W_{ \pm}=\frac{\partial_{\mu} \varphi\left(v^{ \pm}, \mu_{0}\right)}{g^{\prime}\left(v^{ \pm}\right)} .
$$

Applying Lemma 6.2, we infer that $\hat{E}(\gamma, \delta)=\operatorname{det}\left(\hat{a}_{j k}(\gamma, \delta)\right)_{1 \leq j, k \leq 2}$, where

$$
\hat{a}_{j k}(\gamma, \delta)=\left\langle\hat{\mathcal{T}}(\gamma, \delta)\left(x_{j}+\hat{z}_{j}(\gamma)\right), y_{k}\right\rangle_{L^{2}} \quad \text { for all } \quad j, k=1,2 .
$$

We will split each of the entries above in two parts:

$$
\hat{b}_{j k}(\gamma, \delta)=\left\langle\hat{\mathcal{T}}(\gamma, \delta) x_{j}, y_{k}\right\rangle_{L^{2}}, \quad \hat{c}_{j k}(\gamma, \delta)=\left\langle\hat{\mathcal{T}}(\gamma, \delta) \hat{z}_{j}(\gamma), y_{k}\right\rangle_{L^{2}} \quad \text { for all } \quad j, k=1,2 .
$$

We will compute the expansions of $\hat{b}_{j k}$ and $\hat{c}_{j k}, j, k=1,2$, separately. First, we differentiate in (5.20) to compute

$$
\begin{aligned}
\partial_{\delta} \hat{\mathcal{T}}(\gamma, \delta)=\mathcal{L}^{\prime}(\delta) & {\left[\underline{w}+\beta_{1} \hat{\alpha}_{+}(\gamma, \delta) \hat{h}_{+}(\gamma, \delta)+\beta_{2} \hat{\alpha}_{-}(\gamma, \delta) \hat{h}_{-}(\gamma, \delta)\right] } \\
+ & \left(\mathcal{L}(\delta)-\gamma^{2}\right)\left[\beta_{1} \partial_{\delta}\left(\hat{\alpha}_{+} \hat{h}_{+}\right)(\gamma, \delta)+\beta_{2} \partial_{\delta}\left(\hat{\alpha}_{-} \hat{h}_{-}\right)(\gamma, \delta)\right]
\end{aligned}
$$

where

$$
\mathcal{L}^{\prime}(\delta)=\left[\begin{array}{cc}
0 & 0 \\
1 & -\frac{b^{*}}{a^{*}}
\end{array}\right]
$$

In addition, from (4.20), it follows that

$$
\partial_{\delta} \hat{\nu}_{ \pm}(0,0)=0, \quad \partial_{\delta} \hat{\alpha}_{ \pm}(0,0)=\left(\frac{b^{ \pm}}{a^{ \pm}}, 1\right)^{\mathrm{T}}, \quad \partial_{\delta}\left(\hat{\alpha}_{ \pm} \hat{h}_{ \pm}\right)(0,0)=\left(\frac{b^{ \pm}}{a^{ \pm}}, 1\right)^{\mathrm{T}} \chi_{ \pm} .
$$

Since $u^{*}=\varphi\left(v^{*}, \mu_{0}\right)$, from $(2.3)$ and $(7.26)$ we obtain that

$$
\mathcal{L}^{\prime}(0)\left(u_{x}^{*}, v_{x}^{*}\right)^{\mathrm{T}}=(0,0)^{\mathrm{T}}
$$

Using (7.16), (7.17), (7.25) and (7.28) we obtain that

$$
\begin{aligned}
\hat{b}_{11}(\gamma, \delta) & =\hat{b}_{11}(\gamma, 0)+\partial_{\delta} \hat{b}_{11}(\gamma, 0) \delta+\mathcal{O}\left(\delta^{2}\right) \\
& =\left\langle\mathcal{T}(\gamma) x_{1}, y_{1}\right\rangle_{L^{2}}+\delta\left\langle\partial_{\delta} \hat{\mathcal{T}}(\gamma, 0) x_{1}, y_{1}\right\rangle_{L^{2}}+\mathcal{O}\left(\delta^{2}\right) \\
& =-\left(u^{+}-u^{-}\right) \gamma^{2}+\delta\left\langle\mathcal{L}^{\prime}(0)\left(u_{x}^{*}, v_{x}^{*}\right)^{\mathrm{T}},(1,0)^{\mathrm{T}}\right\rangle_{L^{2}}+\mathcal{O}\left(\delta^{2}\right) \\
& =-\left(u^{+}-u^{-}\right) \gamma^{2}+\mathcal{O}\left(\delta^{2}\right) ; \\
\hat{b}_{12}(\gamma, \delta) & =\hat{b}_{12}(\gamma, 0)+\partial_{\delta} \hat{b}_{12}(\gamma, 0) \delta+\mathcal{O}\left(\delta^{2}\right) \\
& =\left\langle\mathcal{T}(\gamma) x_{1}, y_{2}\right\rangle_{L^{2}}+\delta\left\langle\partial_{\delta} \hat{\mathcal{T}}(\gamma, 0) x_{1}, y_{2}\right\rangle_{L^{2}}+\mathcal{O}\left(\delta^{2}\right) \\
& =-\left\|v_{x}^{*}\right\|_{2}^{2} \gamma^{2}+\delta\left\langle\mathcal{L}^{\prime}(0)\left(u_{x}^{*}, v_{x}^{*}\right)^{\mathrm{T}},\left(0, v_{x}^{*}\right)^{\mathrm{T}}\right\rangle_{L^{2}}+\mathcal{O}\left(\delta^{2}\right) \\
& =-\left\|v_{x}^{*}\right\|_{2}^{2} \gamma^{2}+\mathcal{O}\left(\delta^{2}\right) .
\end{aligned}
$$

Next, from Remark 5.5 and (7.27) it follows that

$$
\left\langle\mathcal{L}(0) \partial_{\delta}\left(\hat{\alpha}_{ \pm} \hat{h}_{ \pm}\right)(0,0),(1,0)^{\mathrm{T}}\right\rangle_{L^{2}}=\left\langle\mathcal{L}(0)\left(\frac{b^{ \pm}}{a^{ \pm}}, 1\right)^{\mathrm{T}} \chi_{ \pm},(1,0)^{\mathrm{T}}\right\rangle_{L^{2}}=0
$$




$$
\left\langle\mathcal{L}(0) \partial_{\delta}\left(\hat{\alpha}_{ \pm} \hat{h}_{ \pm}\right)(0,0),\left(0, v_{x}^{*}\right)^{\mathrm{T}}\right\rangle_{L^{2}}=\left\langle\mathcal{L}(0)\left(\frac{b^{ \pm}}{a^{ \pm}}, 1\right)^{\mathrm{T}} \chi_{ \pm},\left(0, v_{x}^{*}\right)^{\mathrm{T}}\right\rangle_{L^{2}}=0
$$

From (7.25), (7.26) and (7.30) we obtain that

$$
\begin{aligned}
\partial_{\delta} \hat{b}_{21}(\gamma, 0)= & \partial_{\delta} \hat{b}_{21}(0,0)+\mathcal{O}(\gamma)=\left\langle\partial_{\delta} \hat{\mathcal{T}}(0,0) x_{2}, y_{1}\right\rangle_{L^{2}}+\mathcal{O}(\gamma) \\
= & \left\langle\mathcal{L}(0)\left[W_{+} \partial_{\delta}\left(\hat{\alpha}_{+} \hat{h}_{+}\right)(0,0)+W_{-} \partial_{\delta}\left(\hat{\alpha}_{-} \hat{h}_{-}\right)(0,0)\right],(1,0)^{\mathrm{T}}\right\rangle_{L^{2}} \\
& \quad+\left\langle\mathcal{L}^{\prime}(0)(U, 0)^{\mathrm{T}},(1,0)^{\mathrm{T}}\right\rangle_{L^{2}}+\mathcal{O}(\gamma) \\
= & \left\langle(0, U)^{\mathrm{T}},(1,0)^{\mathrm{T}}\right\rangle_{L^{2}}+\mathcal{O}(\gamma) \\
= & \mathcal{O}(\gamma) ; \\
\partial_{\delta} \hat{b}_{22}(\gamma, 0)= & \partial_{\delta} \hat{b}_{22}(0,0)+\mathcal{O}(\gamma)=\left\langle\partial_{\delta} \hat{\mathcal{T}}(0,0) x_{2}, y_{2}\right\rangle_{L^{2}}+\mathcal{O}(\gamma) \\
= & \left\langle\mathcal{L}(0)\left[W_{+} \partial_{\delta}\left(\hat{\alpha}_{+} \hat{h}_{+}\right)(0,0)+W_{-} \partial_{\delta}\left(\hat{\alpha}_{-} \hat{h}_{-}\right)(0,0)\right],\left(0, v_{x}^{*}\right)^{\mathrm{T}}\right\rangle_{L^{2}} \\
& \quad+\left\langle\mathcal{L}^{\prime}(0)(U, 0)^{\mathrm{T}},\left(0, v_{x}^{*}\right)^{\mathrm{T}}\right\rangle_{L^{2}}+\mathcal{O}(\gamma) \\
= & \left\langle(0, U)^{\mathrm{T}},\left(0, v_{x}^{*}\right)^{\mathrm{T}}\right\rangle_{L^{2}}+\mathcal{O}(\gamma) \\
= & \int_{v^{-}}^{v^{+}} \partial_{\mu} \varphi\left(v, \mu_{0}\right) \mathrm{d} v+\mathcal{O}(\gamma) .
\end{aligned}
$$

Using (7.18), (7.19) and (7.31) we conclude that

$$
\begin{aligned}
\hat{b}_{21}(\gamma, \delta) & =\hat{b}_{21}(\gamma, 0)+\partial_{\delta} \hat{b}_{21}(\gamma, 0) \delta+\mathcal{O}\left(\delta^{2}\right) \\
& =-\left[\left(a^{+}\right)^{1 / 2} \partial_{\mu} \varphi\left(v^{+}, \mu_{0}\right)+\left(a^{-}\right)^{1 / 2} \partial_{\mu} \varphi\left(v^{-}, \mu_{0}\right)\right] \gamma+\mathcal{O}\left(\gamma^{2}\right)+\mathcal{O}(\gamma \delta)+\mathcal{O}\left(\delta^{2}\right) \\
\hat{b}_{22}(\gamma, \delta) & =\hat{b}_{22}(\gamma, 0)+\partial_{\delta} \hat{b}_{22}(\gamma, 0) \delta+\mathcal{O}\left(\delta^{2}\right) \\
& =\left(\int_{v^{-}}^{v^{+}} \partial_{\mu} \varphi\left(v, \mu_{0}\right) \mathrm{d} v\right) \delta+\mathcal{O}\left(\gamma^{2}\right)+\mathcal{O}(\gamma \delta)+\mathcal{O}\left(\delta^{2}\right) .
\end{aligned}
$$

Since $\hat{z}_{j}(\gamma, \delta) \in H_{\eta}^{2}\left(\mathbb{R}, \mathbb{C}^{2}\right) \times \mathbb{C}^{2}$ for all $\gamma, \delta$ in a neighborhood of 0 and $j=1,2$, we infer that

$$
\begin{aligned}
\hat{c}_{j k}(\gamma, \delta) & =\left\langle\hat{\mathcal{T}}(0,0) \hat{z}_{j}(\gamma, \delta), y_{k}\right\rangle_{L^{2}}+\left\langle(\hat{\mathcal{T}}(\gamma, \delta)-\hat{\mathcal{T}}(0,0)) \hat{z}_{j}(\gamma, \delta), y_{k}\right\rangle_{L^{2}} \\
& =\left\langle\hat{z}_{j}(\gamma, \delta), \hat{\mathcal{T}}(0,0)^{*} y_{k}\right\rangle_{L^{2}}+\left\langle(\hat{\mathcal{T}}(\gamma, \delta)-\hat{\mathcal{T}}(0,0)) \hat{z}_{j}(\gamma, \delta), y_{k}\right\rangle_{L^{2}} \\
& =\left\langle(\hat{\mathcal{T}}(\gamma, \delta)-\hat{\mathcal{T}}(0,0)) \hat{z}_{j}(\gamma, \delta), y_{k}\right\rangle_{L^{2}} .
\end{aligned}
$$

In addition, from Lemma 6.2 and since $z_{1}^{\prime}(0)=0$ we have that

$$
\begin{aligned}
& \hat{z}_{1}(\gamma, \delta)=\hat{z}_{1}(\gamma, 0)+\mathcal{O}(\delta)=z_{1}(\gamma)+\mathcal{O}(\delta)=\mathcal{O}\left(\gamma^{2}\right)+\mathcal{O}(\delta) \\
& \hat{z}_{2}(\gamma, \delta)=\hat{z}_{2}(\gamma, 0)+\mathcal{O}(\delta)=z_{2}(\gamma)+\mathcal{O}(\delta)=\mathcal{O}(\gamma)+\mathcal{O}(\delta) .
\end{aligned}
$$

From (7.33) and (7.34)

$$
\hat{c}_{1 k}(\gamma, \delta)=\mathcal{O}\left(\gamma^{3}\right)+\mathcal{O}(\gamma \delta)+\mathcal{O}\left(\gamma^{2} \delta\right)+\mathcal{O}\left(\delta^{2}\right), \quad \hat{c}_{2 k}(\gamma, \delta)=\mathcal{O}\left(\gamma^{2}\right)+\mathcal{O}(\gamma \delta)+\mathcal{O}\left(\delta^{2}\right), \quad k=1,2 .
$$

Next, we will show that the coefficient of $\gamma \delta$ in the expansion of $\hat{c}_{12}(\gamma, \delta)$ is 0 . The latter coefficient, denoted $c_{0}$, is given by

$$
c_{0}=\left\langle\partial_{\gamma} \hat{\mathcal{T}}(0,0) \partial_{\delta} \hat{z}_{1}(0,0),\left(0, v_{x}^{*}\right)^{\mathrm{T}}\right\rangle_{L^{2}}=\left\langle\mathcal{T}^{\prime}(0) \partial_{\delta} \hat{z}_{1}(0,0),\left(0, v_{x}^{*}\right)^{\mathrm{T}}\right\rangle_{L^{2}}
$$




$$
=\left\langle\partial_{\delta} \hat{z}_{1}(0,0), \mathcal{T}^{\prime}(0)^{*}\left(0, v_{x}^{*}\right)^{\mathrm{T}}\right\rangle_{L^{2}}
$$

From Remark 5.5 we have that

$$
\left\langle\mathcal{L}(0)\left(\alpha_{ \pm}(0) h_{ \pm}(0)\right),\left(0, v_{x}^{*}\right)^{\mathrm{T}}\right\rangle_{L^{2}}=0
$$

From (7.7) it follows that $\mathcal{T}^{\prime}(0)\left(0, v_{x}^{*}\right)^{\mathrm{T}}=0$, proving via $(7.36)$ that $c_{0}=0$. We infer that

$$
\begin{aligned}
& \hat{c}_{11}(\gamma, \delta)=\mathcal{O}\left(\gamma^{3}\right)+\mathcal{O}(\gamma \delta)+\mathcal{O}\left(\delta^{2}\right) \\
& \hat{c}_{12}(\gamma, \delta)=\mathcal{O}\left(\gamma^{3}\right)+\mathcal{O}\left(\gamma^{2} \delta\right)+\mathcal{O}\left(\delta^{2}\right) \\
& \hat{c}_{2 k}(\gamma, \delta)=\mathcal{O}\left(\gamma^{2}\right)+\mathcal{O}(\gamma \delta)+\mathcal{O}\left(\delta^{2}\right), \quad k=1,2 .
\end{aligned}
$$

Collecting the results from (7.29), (7.32) and (7.38) we conclude that

$$
\begin{aligned}
& \hat{a}_{11}(\gamma, \delta)=-\left(u^{+}-u^{-}\right) \gamma^{2}+\mathcal{O}\left(\gamma^{3}\right)+\mathcal{O}(\gamma \delta)+\mathcal{O}\left(\delta^{2}\right) \\
& \hat{a}_{12}(\gamma, \delta)=-\left\|v_{x}^{*}\right\|_{2}^{2} \gamma^{2}+\mathcal{O}\left(\gamma^{3}\right)+\mathcal{O}\left(\gamma^{2} \delta\right)+\mathcal{O}\left(\delta^{2}\right) \\
& \hat{a}_{21}(\gamma, \delta)=-\left[\left(a^{+}\right)^{1 / 2} \partial_{\mu} \varphi\left(v^{+}, \mu_{0}\right)+\left(a^{-}\right)^{1 / 2} \partial_{\mu} \varphi\left(v^{-}, \mu_{0}\right)\right] \gamma+\mathcal{O}\left(\gamma^{2}\right)+\mathcal{O}(\gamma \delta)+\mathcal{O}\left(\delta^{2}\right) \\
& \hat{a}_{22}(\gamma, \delta)=\left(\int_{v^{-}}^{v^{+}} \partial_{\mu} \varphi\left(v, \mu_{0}\right) \mathrm{d} v\right) \delta+\mathcal{O}\left(\gamma^{2}\right)+\mathcal{O}(\gamma \delta)+\mathcal{O}\left(\delta^{2}\right),
\end{aligned}
$$

proving the lemma.

Lemma 7.5. Under the assumptions of Theorem 1.1, let $\gamma_{*}(\delta)$ be a simple root of $\hat{E}(\gamma, \delta)$ and $\operatorname{Re} \gamma_{*}>0$. Then $\lambda_{*}(\delta)=\gamma_{*}^{2}(\delta)$ is an algebraically simple eigenvalue of $\mathcal{L}(\delta)$.

Proof. Let $\hat{A}(\gamma, \delta)=\left(\hat{a}_{j k}(\gamma, \delta)\right)_{1 \leq j, k \leq 2}$, be the matrix with entries $\hat{a}_{j, k}(\gamma, \delta)$ defined in (7.23). Since $\operatorname{det} \hat{A}\left(\gamma_{*}(\delta), \delta\right)=\hat{E}\left(\gamma_{*}(\delta), \delta\right)=0$, we have that $\hat{A}\left(\gamma_{*}(\delta), \delta\right)$ possesses a kernel. In other words, $\gamma_{*}(\delta)$ is a isolated, generalized eigenvalue of the generalized eigenvalue problem associated with $\hat{A}\left(\gamma_{*}(\delta), \delta\right)$. From [6, Theorem XI.9.1] we know that the algebraic multiplicity of this generalized eigenvalue is equal to

$$
\frac{1}{2 \pi \mathrm{i}} \operatorname{tr}\left(\int_{\Gamma_{*}(\delta)} \partial_{\gamma} \hat{A}(\gamma, \delta) \hat{A}(\gamma, \delta)^{-1} \mathrm{~d} \gamma\right)
$$

where $\Gamma_{*}(\delta)$ is a small circular contour centered at $\gamma_{*}(\delta)$, anti-clockwise oriented. On the other hand, one can easily check that

$$
\operatorname{tr}\left(\partial_{\gamma} \hat{A}(\gamma, \delta) \hat{A}(\gamma, \delta)^{-1}\right)=\frac{\partial_{\gamma} \hat{E}(\gamma, \delta)}{\hat{E}(\gamma, \delta)} .
$$

Since $\gamma_{*}(\delta)$ is a simple root of $\hat{E}(\cdot, \delta)$, from (7.39) and (7.40) we conclude that the algebraic multiplicity of $\gamma_{*}(\delta)$ as to $\hat{A}(\gamma(\delta), \delta)$ is 1 . Thus, the meromorphic function $\hat{A}(\cdot, \delta)^{-1}$ has a 1 -dimensional pole of order 1 at $\gamma_{*}(\delta)$. From Lemma 6.1 and Lemma 6.2 we obtain that the meromorphic function $\hat{\mathcal{T}}(\cdot, \delta)^{-1}$ has a 1 -dimensional pole of order 1 at $\gamma_{*}(\delta)$. Moreover, we have that

$$
\left(\mathcal{L}(\delta)-\gamma^{2}\right)^{-1}\left(\begin{array}{l}
f_{1} \\
f_{2}
\end{array}\right)=\hat{V}(\gamma, \delta) \hat{\mathcal{T}}(\gamma, \delta)^{-1}\left(\begin{array}{l}
f_{1} \\
f_{2}
\end{array}\right)
$$


for all $\left(\begin{array}{l}f_{1} \\ f_{2}\end{array}\right) \in L_{\eta}^{2}\left(\mathbb{R}, \mathbb{C}^{2}\right)$, where $\hat{V}(\gamma, \delta): H_{\eta}^{2}\left(\mathbb{R}, \mathbb{C}^{2}\right) \times \mathbb{C}^{2} \rightarrow H^{2}\left(\mathbb{R}, \mathbb{C}^{2}\right)$ is defined by

$$
\hat{V}(\gamma, \delta)\left(\underline{w}, \beta_{1}, \beta_{2}\right)^{\mathrm{T}}=\underline{w}+\beta_{1} \hat{\alpha}_{+}(\gamma, \delta) \hat{h}_{+}(\gamma, \delta)+\beta_{2} \hat{\alpha}_{-}(\gamma, \delta) \hat{h}_{-}(\gamma, \delta) .
$$

Since the functions $\hat{\alpha}_{ \pm}(\cdot, \delta)$ and $\hat{h}_{ \pm}(\cdot, \delta)$ are holomorphic, we infer that the function $\hat{V}(\cdot, \delta)$ is holomorphic. This implies that $\left(\mathcal{L}(\delta)-\gamma^{2}\right)^{-1}$ has a simple 1-dimensional pole at $\gamma=\gamma_{*}(\delta)$ when considered on $L_{\eta}^{2}\left(\mathbb{R}, \mathbb{C}^{2}\right)$. Using again the fact that $L_{\eta}^{2}\left(\mathbb{R}, \mathbb{C}^{2}\right)$ is dense in $L^{2}\left(\mathbb{R}, \mathbb{C}^{2}\right)$, we conclude that the same property holds for the resolvent on $L^{2}\left(\mathbb{R}, \mathbb{C}^{2}\right)$. Since $\gamma_{*}(\delta) \neq 0$, the transformation from $\gamma$ to $\lambda=\sqrt{\gamma}$ is analytic in a neighborhood of $\gamma_{*}(\delta)$ and we conclude that the resolvent $(\mathcal{L}(\delta)-\lambda)^{-1}$ also has a simple 1-dimensional pole in $\lambda$, which proves the lemma.

Remark 7.6. Lemma 7.5 can readily be generalized to show that the order of roots of $\hat{E}$ coincides with algebraic multiplicities of eigenvalues $\lambda$ in the point spectrum of $\mathcal{L}$, that is, in the region where $\mathcal{L}-\lambda$ is Fredholm with index 0.

\section{Proof of Theorem 1.1}

In this section we prove the main result of this paper on the stability of layers. We begin by showing that (1.1) has a formal gradient structure in the case when $\delta>0, b\left(u^{*}\right) \geq b_{0}>0$. We will use this result to exclude Hopf bifurcations during the homotopy.

Remark 8.1. If $\delta>0, b\left(u^{*}\right) \geq b_{0}>0$, then (1.1) has the formal gradient structure

$$
\frac{\mathrm{d}}{\mathrm{d} t}(u, v)^{\mathrm{T}}=-\mathcal{M}(u) \nabla_{L^{2}} \mathcal{E}(u, v),
$$

where

$$
\begin{aligned}
& \mathcal{E}(u, v)=\int_{\mathbb{R}}\left(\frac{1}{2 \delta}\left|v_{x}\right|^{2}-\frac{1}{\delta} G(v)+J(u)-u v\right) \mathrm{d} x, \quad G^{\prime}=g, j^{\prime}=\frac{a}{b}, J^{\prime}=j ; \\
& \mathcal{M}(u): H^{2}(\mathbb{R}, \mathbb{C}) \times L^{2}(\mathbb{R}, \mathbb{C}) \rightarrow L^{2}\left(\mathbb{R}, \mathbb{C}^{2}\right), \quad \mathcal{M}(u)=\left[\begin{array}{cc}
-\partial_{x}\left(b(u) \partial_{x}\right) & 0 \\
0 & \delta
\end{array}\right] .
\end{aligned}
$$

We note that

$$
\mathcal{M}(u)^{*}=\mathcal{M}(u), \quad \mathcal{M}(u) \geq 0 \quad \text { for all } \quad u \in \mathbb{R} .
$$

Lemma 8.2. If $\delta_{0}>0, b\left(u^{*}\right) \geq b_{0}>0$, there are no Hopf bifurcations during the homotopy, that is, the eigenvalues of $\mathcal{L}(\delta)$ are real.

Proof. From (8.1) we have that $\mathcal{L}(\delta)=-\mathcal{M N}$, where $\mathcal{M}=\mathcal{M}\left(u^{*}\right)$ and $\mathcal{N}=D^{2} \mathcal{E}\left(u^{*}, v^{*}\right)$. From (8.4) it follows that

$$
\mathcal{M}^{*}=\mathcal{M} \geq 0
$$

In addition, one readily checks that

$$
\mathcal{N}^{*}=\mathcal{N}, \quad \operatorname{ker} \mathcal{M}=\{0\} .
$$


Next, we define $\mathcal{Y}:=\operatorname{im} \mathcal{M}$ and the scalar product on $\mathcal{Y}$ :

$$
\langle u, v\rangle_{\mathcal{Y}}=\left\langle\mathcal{M}^{-1} u, v\right\rangle_{L^{2}} \quad \text { for } \quad u, v \in \mathcal{Y} .
$$

Let $\lambda \in \mathbb{C} \backslash\{0\}, u_{\lambda} \in H^{2}\left(\mathbb{R}, \mathbb{C}^{2}\right), u_{\lambda} \neq 0$ such that $\mathcal{L} u_{\lambda}=\lambda u_{\lambda}$. From the decomposition of $\mathcal{L}$ it follows that $\lambda u_{\lambda}=-\mathcal{M N} u_{\lambda}$, which shows that $u \in \mathcal{Y}$. From (8.6) we infer that

$$
\begin{aligned}
\lambda\left\|u_{\lambda}\right\|_{\mathcal{Y}}^{2} & =\left\langle\mathcal{L} u_{\lambda}, u_{\lambda}\right\rangle \mathcal{Y}=\left\langle\mathcal{M}^{-1} \mathcal{L} u_{\lambda}, u_{\lambda}\right\rangle_{L^{2}}=-\left\langle\mathcal{N} u_{\lambda}, u_{\lambda}\right\rangle_{L^{2}} \\
& =-\left\langle u_{\lambda}, \mathcal{N} u_{\lambda}\right\rangle_{L^{2}}=-\left\langle\mathcal{M} \mathcal{M}^{-1} u_{\lambda}, \mathcal{N} u_{\lambda}\right\rangle_{L^{2}}=\left\langle\mathcal{M}^{-1} u_{\lambda},-\mathcal{M N} u_{\lambda}\right\rangle_{L^{2}} \\
& =\left\langle\mathcal{M}^{-1} u_{\lambda}, \mathcal{L} u_{\lambda}\right\rangle_{L^{2}}=\left\langle\mathcal{M}^{-1} u_{\lambda}, \lambda u_{\lambda}\right\rangle_{L^{2}}=\bar{\lambda}\left\|u_{\lambda}\right\|_{\mathcal{Y}}^{2}
\end{aligned}
$$

Since $u_{\lambda} \neq 0$ it follows that $\lambda=\bar{\lambda}$, which implies that $\lambda \in \mathbb{R}$, proving the lemma.

Proof of Theorem 1.1. First, we focus on the proof of (i). We note that from the hypothesis we have that $b\left(u^{*}\right) \geq b_{0}>0$. Indeed, $\left(u^{+}-u^{-}\right)\left(v^{+}-v^{-}\right)>0$ implies that $u^{*}$ is nonconstant. Moreover, $u^{*}=\varphi\left(v^{*}, \mu_{0}\right)$, and since $\varphi\left(\cdot, \mu_{0}\right)$ is the solution of an autonomous first order differential equation, we have that $u^{*}$ is different from any zero of $b$. Thus, $b\left(u^{*}\right) \neq 0$ and since $b\left(u^{*}\right)=a\left(u^{*}\right) \frac{u_{x}^{*}}{v_{x}^{*}} \geq 0$ we conclude that $b\left(u^{*}\right)>0$. Finally, since $u^{*}$ is bounded we have that $b_{0}=\inf \left[b\left(u^{*}\right)\right]>0$.

Given that $g^{\prime}\left(v^{ \pm}\right)<0$, from Lemma 3.4 we have that the essential spectrum of $\mathcal{L}$ is stable. Next, we look at the homotopy defined in (4.1) and from Lemma 4.2 we know that the essential spectrum of $\mathcal{L}(\delta)$ is stable for all $\delta \in\left[0, \delta_{0}\right]$. In addition, from Lemma 4.3 we know that the layer is stable at the end of the homotopy, for $\delta=0$. We now define $i(\delta)$ to be the number of eigenvalues in $\operatorname{Re} \lambda>0$, counted with algebraic multiplicity. We have $i(0)=0$. Next, notice that the expansions in Lemma 7.1 and Remark 7.2 guarantee that $E(\gamma) \neq 0$ for $\gamma$ small, nonzero, and $\delta \neq 0$, so that there is a neighborhood of the origin $\{|\lambda| \leq \varepsilon(\delta), \operatorname{Re} \lambda \geq 0\} \backslash\{0\}$ that does not contain any eigenvalues for any $\delta \in\left(0, \delta_{0}\right]$. Lemma 5.7 ensures that we can choose $\varepsilon(\delta) \geq \varepsilon_{*}>0$, uniformly in $\delta \geq \delta_{*}>0$ for any $\delta_{*}>0$. For $\delta \geq 0$, small, we use the expansion from Lemma 7.3. Since $b\left(u^{*}\right) \geq b_{0}>0$ we have that $c_{2} \leq 0$. Since $c_{3}<0$ and $\delta \geq 0$, we find that $\varepsilon(\delta)>\varepsilon_{*}$ can be chosen uniformly positive for all $\delta \in\left[0, \delta_{0}\right]$.

Lemma 8.2 guarantees that the spectrum does not intersect the imaginary axis $i \mathbb{R} \backslash\{0\}$ for any $\delta \in\left[0, \delta_{0}\right]$. We can therefore find a closed curve $\Gamma$ in the resolvent set,

$$
\Gamma=\left\{|\lambda|=\varepsilon_{*}, \operatorname{Re} \lambda \geq 0\right\} \cup\{|\lambda|=R, \operatorname{Re} \lambda \geq 0\} \cup \mathrm{i}\left[\varepsilon_{*}, R\right] \cup \mathrm{i}\left[-R,-\varepsilon_{*}\right],
$$

$R$ sufficiently large, so that $\operatorname{Re} \lambda>0, \lambda \in \sigma(\mathcal{L}(\delta))$ implies $\lambda \in \operatorname{int}(\Gamma)$. The Dunford integral

$$
P(\delta)=\frac{1}{2 \pi \mathrm{i}} \int_{\Gamma}(\lambda-\mathcal{L}(\delta))^{-1} \mathrm{~d} \lambda,
$$

then gives the spectral projection on all eigenvalues with positive real part. Since $P(\delta)$ is continuous in $\delta$, we conclude that $\operatorname{dim} \operatorname{Im}(P(\delta))$ is constant. Since $P(0)=0$, this proves (i) in Theorem 1.1.

We next prove (ii). Again, the essential spectrum is stable so that it is sufficient to track point spectrum during the homotopy. The expansion of $\hat{E}(\gamma, \delta)$ in Lemma 7.4 now yields for each 
small $\delta$ a unique simple root $\gamma=\gamma_{*}(\delta)$ of $\hat{E}(\gamma, \delta)=0$ in $\gamma>0$, which corresponds to a unique eigenvalue $\lambda_{*}(\delta)$ in $\operatorname{Re} \lambda>0$. Since we have $\partial_{\gamma} \hat{E}(\gamma, \delta) \neq 0$ at this eigenvalue, Lemma 7.5 shows that this eigenvalue is in fact algebraically simple.

We next consider $i(\delta)$, the number of eigenvalues counted with multiplicity in $\left[\varepsilon_{*}, R\right]$. Choosing $\delta_{*}$ and $\varepsilon_{*}$ sufficiently small, we know that $\varepsilon_{*}$ is not in the spectrum for all $\delta$ between 0 and $\delta_{0}$ with $|\delta| \geq \delta_{*}$ and $i\left(\delta_{*}\right)=1$. Also, $R$ sufficiently large is in the resolvent set for all $\delta$ between 0 and $\delta_{0}$. We claim that this implies that $i(\delta)$ is odd for all $\delta$ between 0 and $\delta_{0}$ with $|\delta| \geq \delta_{*}$. To see this, notice first that $i(\delta)$ is finite since the operator $\mathcal{L}(\delta)-\lambda$ is Fredholm with index zero for $\lambda>0$. Since $\varepsilon_{*}$ and $R$ are in the resolvent set, $i(\delta)$ can only change when eigenvalues become complex, which leads to a change in multiplicity by even integers. This can readily be seen by counting eigenvalues in $\left[\varepsilon_{*}, R\right]$, as well as eigenvalues in $\left\{\operatorname{Re} \lambda \in\left[\varepsilon_{*}, R\right], \operatorname{Im} \lambda>0\right\}$ near points $\delta$ where $i(\delta)$ changes, while exploiting that algebraic multiplicities of $\lambda$ and $\bar{\lambda}$ coincide. In summary, we showed that $i\left(\delta_{0}\right)$ is odd, which implies in particular that $i\left(\delta_{0}\right) \neq 0$, so that there exists an eigenvalue $\lambda>0$. This proves (ii).

Assertion (iii) follows directly from Lemma 2.2, Lemma 3.4, Lemma 4.2 and Lemma 4.4.

\section{Perturbations of stable layers}

In this section we prove Theorem 1.2. Assertions (i) and (ii) where proved in Lemma 2.3 and Lemma 2.6, respectively. To prove assertion (iii), first consider assume that $u^{+} \neq u^{-}$. Robustness of the layer, assertion (i), then guarantees that the nearby layer has the same signs of $\left(u_{+}-u_{-}\right)\left(v_{+}-v_{-}\right) \delta_{0}$, which, using Theorem 1.1, (i)-(ii) guarantees that the nearby layer shares the same stability properties. On the other hand, when $u_{+}=u_{-}$, we can perturb $b \mapsto b+\varepsilon$ to achieve $u^{+}-u^{-}=K \varepsilon+\mathrm{O}\left(\varepsilon^{2}\right)$ with $K \neq 0$, according to Lemma 2.5, which shows that $\left(u_{+}-u_{-}\right)\left(v_{+}-v_{-}\right) \delta_{0}$ changes sign when viewed as a function of $\varepsilon$. Again invoking Theorem 1.1, (i)-(ii), we find that the stability of the layer changes as $\varepsilon$ changes sign. Similarly, the last assertion (iv) is a consequence of the sign change of $\left(u_{+}-u_{-}\right)\left(v_{+}-v_{-}\right) \delta$ as $\delta$ changes sign. This concludes the proof of Theorem 1.2.

\section{References}

[1] G. Caginalp, An analysis of a phase field model of a free boundary, Arch. Ration. Mech. Anal. 92 (1986), 205-245.

[2] P. Coullet, J. Lega, B. Houchmanzadeh, and J. Lajzerowicz, Breaking chirality in nonequilibrium systems, Phys. Rev. Lett. 65 (1990), 1352-1355.

[3] M. Droz, Recent theoretical developments on the formation of Liesegang patterns, J. Stat. Phys. 101 (2000), 509-519.

[4] P. C. Fife, Pattern formation in gradient systems, Handbook of dynamical systems, 2 (2002), 677-722, North-Holland, Amsterdam. 
[5] R. Goh, S. Mesuro, and A. Scheel, Coherent structures in reaction-diffusion models for precipitation, Special volume on "Precipitation patterns in reaction-diffusion systems", Research Signpost, 2010, 73-93.

[6] I. Gohberg, S. Goldberg, M. Kaashoek, Classes of linear operators, Vol. 1, Birkhauser, 1990.

[7] P. S. Hagan, The instability of nonmonotonic wave solutions of parabolic equations, Stud. Appl. Math. 64 (1981), 57-88.

[8] A. Hagberg and E. Meron, Pattern formation in non-gradient reaction-diffusion systems: the effects of front bifurcations, Nonlinearity, 7 (1994), 805-835.

[9] D. Henry, Geometric Theory of Semilinear Parabolic Equations, Lect. Notes Math. 840 (1981), Springer-Verlag, New York.

[10] D. Hilhorst, R. van der Hout, M. Mimura, and I. Ohnishi, Fast reaction limits and Liesegang bands. Free boundary problems, 241-250, Internat. Ser. Numer. Math. 154 (2007), Birkhäuser, Basel.

[11] T. Hillen and K. Painter, A user's guide to PDE models for chemotaxis, J. Math. Biol. 58, (2008), 183-217.

[12] T. Hillen and K. Painter, Volume-filling and quorum-sensing in models for chemosensitive movement, Can. Appl. Math. Q. 10 (2002), 501-543.

[13] H. Ikeda, M. Mimura, and Y. Nishiura, Global bifurcation phenomena of traveling wave solutions for some bistable reaction-diffusion systems, Nonlinear Anal. 13 (1989), 507-526.

[14] A. Jilkine, L. Keshet, and Y. Mori, Wave-pinning and cell polarity from a bistable reactiondiffusion system, Bioph. J. 94 (2008), 3684-3697.

[15] E. Keller and L. Segel, Initiation of slime mold aggregation viewed as an instability, J. Theor. Biol. 26 (1970), 399-415.

[16] J. Keller and S. Rubinow, Recurrent precipitation and Liesegang rings, J. Chem. Phys. 74 (1981), 5000-5007.

[17] A. Lunardi, Analytic Semigroups and Optimal Regularity in Parabolic Problems, Progress in Nonlinear Differential Equations and their Applications 16 (1995), Birkhäuser Verlag, Basel.

[18] J. Maddocks, Restricted quadratic forms and their application to bifurcation and stability in constrained variational principles, SIAM J. Math. Anal. 16 (1985), 47-68.

[19] Y. Morita and T. Ogawa, Stability and bifurcation of nonconstant solutions to a reactiondiffusion system with conservation of mass, Nonlinearity 23 (2010), 1387-1411.

[20] K. J. Palmer, Exponential dichotomies and transversal homoclinic points, J. Diff. Eq. 55 (1984), 225-256. 
[21] K. J. Palmer, Exponential dichotomies and Fredholm operators, Proc. Amer. Math. Soc. 104 (1988), 149-156.

[22] A. Pogan and A. Scheel, Instability of Spikes in the Presence of Conservation Laws, to appear, Z. Angew. Math. Phys. 61 (2010), 979-998.

[23] A. Pogan and A. Scheel, Instability of radially-symmetric spikes in systems with a conserved quantity, Fields Inst. Comm., to appear.

[24] A. Pogan and A. Scheel, Fredholm properties of radially symmetric, second order differential operators, Int. J. Dyn. Sys. Diff. Eqns., to appear.

[25] J. Rinzel and D. Terman, Propagation phenomena in a bistable reaction-diffusion system, SIAM J. Appl. Math. 42 (1982), 1111-1137.

[26] B. Sandstede and A. Scheel, Relative Morse indices, Fredholm indices, and group velocities, Discr. Cont. Dynam. Syst. 20 (2008), 139-158.

[27] A. Scheel, Robustness of Liesegang patterns, Nonlinearity 22 (2009), 457-483. 